\title{
BMSAP
}

Bulletins et mémoires de la Société d'Anthropologie de Paris

BMSAP

$33(2) \mid 2021$

Varia

\section{Entre peste et famine : caractérisation d'une crise de mortalité par l'étude de trois sépultures multiples du site de Kutná Hora - Sedlec (République tchèque, $\mathrm{XIV}^{\mathrm{e}}$ siècle)}

Between plague and famine: characterization of a mortality crisis through the study of three mass graves from the Kutná Hora-Sedlec site (Czech Republic, $14^{\text {th }}$ century)

Auxane de Lépinau, Dominique Castex, Hana Brzobohatá, Jan Frolík, Filip Velímský, Jaroslav Brůžek, Petr Velemínský et Sacha Kacki

\section{(2) OpenEdition}

Journals

Édition électronique

URL : https://journals.openedition.org/bmsap/7664

DOI : 10.4000/bmsap.7664

ISSN : $1777-5469$

Éditeur

Société d'Anthropologie de Paris

Référence électronique

Auxane de Lépinau, Dominique Castex, Hana Brzobohatá, Jan Frolík, Filip Velímský, Jaroslav Brůžek, Petr Velemínský et Sacha Kacki, «Entre peste et famine : caractérisation d'une crise de mortalité par l'étude de trois sépultures multiples du site de Kutná Hora - Sedlec (République tchèque, XIVe siècle) ", Bulletins et mémoires de la Société d'Anthropologie de Paris [En ligne], 33 (2) | 2021, mis en ligne le 20 juillet 2021, consulté le 29 octobre 2021. URL : http://journals.openedition.org/bmsap/7664 ; DOl : https://doi.org/10.4000/bmsap.7664

\section{(c) $(1)$}

Les contenus des Bulletins et mémoires de la Société d'Anthropologie de Paris sont mis à disposition selon les termes de la licence Creative Commons Attribution-NonCommercial-NoDerivatives 4.0 International License. 


\title{
Entre peste et famine : caractérisation d'une crise de mortalité par l'étude de trois sépultures multiples du site de Kutná Hora - Sedlec (République tchèque, XIV ${ }^{\mathrm{e}}$ siècle)
}

\author{
Between plague and famine: characterization of a mortality crisis through the study \\ of three mass graves from the Kutná Hora - Sedlec site (Czech Republic, $14^{\text {th }}$ century)
}

Auxane de Lépinau ${ }^{1}$, Dominique Castex ${ }^{1}$, Hana Brzobohatá (D)2 , Jan Frolík (iD)²,

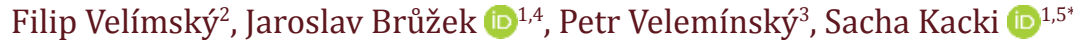

1. PACEA, UMR 5199, CNRS - Université de Bordeaux, F-33600 Pessac, France.

2. Institute of Archaeology of the Czech Academy of Sciences, Letenská 4, 11801 Prague 1, Czech Republic.

3. Department of Anthropology, Národní muzeum, Cirkusová 1740, 19300 Prague 9 - Horní Počernice, Czech Republic.

4. Department of Anthropology and Human Genetics, Faculty of Science, Charles University, 12800 Prague 2, Czech Republic.

5. Department of Archaeology, Durham University, Durham DH1 3LE, United Kingdom.

* sacha.kacki@u-bordeaux.fr

Reçu : 10 novembre 2020 ; accepté : 17 mars 2021

Bulletins et Mémoires de la Société d'Anthropologie de Paris

Résumé - Les derniers siècles du Moyen Âge ont été marqués par des crises épidémiques et frumentaires récurrentes, dont certaines ont entrainé des vagues de mortalité telles qu'elles ont nécessité la création de sépultures de masse et de cimetières d'urgence. Si ces sépultures et ces ensembles funéraires ont souvent fait l'objet d'études anthropologiques, rares sont celles qui se sont appliquées à évaluer les caractéristiques paléobiologiques distinguant les deux types de crises. Le présent article explore cette question à travers l'étude anthropologique et paléopathologique de trois sépultures multiples du XIV ${ }^{\mathfrak{e}}$ siècle mises au jour à Kutná Hora - Sedlec (République tchèque), que des données historiques permettent de relier soit à un épisode de famine, soit à la Peste noire. Les données acquises pour les 68 squelettes exhumés de ces tombes sont comparées à celles obtenues pour un échantillon de 284 squelettes issus d'un cimetière médiéval local (Cathédrale de Sedlec), ainsi qu'à un ensemble de données de référence documentant la mortalité par peste et par famine. Les résultats montrent des différences de composition par âge, de caractéristiques métriques et d'état sanitaire préexistant entre les deux séries tchèques. L'échantillon issu des sépultures multiples présente un profil de mortalité avant 20 ans, des valeurs pour certains indices démographiques et des caractéristiques paléopathologiques qui le rapprochent davantage des données de référence d'une mortalité par peste, ce qui conduit à privilégier l'hypothèse de sa relation avec la Peste noire. Si un diagnostic de certitude nécessitera des analyses complémentaires, cette étude fournit d'ores et déjà des indications sur les critères discriminants entre peste et famine et ouvre la voie à de futures recherches sur les épidémies et crises de subsistance du passé.
Mots clés - crises de mortalité, Peste noire, famine, paléodémographie, démographie historique, indicateurs de stress non spécifiques

\begin{abstract}
The later centuries of the Middle Ages were a time of recurrent epidemics and famines, some of which caused such huge mortality that mass graves and emergency burial grounds had to be created. Several anthropological studies have investigated these graves and cemeteries, but only a few have attempted to assess the bioarchaeological features that could distinguish between these two types of crisis. Our study tackles this question through an anthropological and palaeopathological study of three $14^{\text {th }}$ century mass graves at Kutná Hora - Sedlec (Czech Republic), for which historical data support a relationship with either a famine or the Black Death. Data on the 68 skeletons recovered from these graves were compared with data on 284 skeletons from a local mediaeval cemetery (Sedlec Cathedral) and with a set of reference data documenting plague and famine mortality. The results reveal differences in age structure, osteometrics and pre-existing health status between the catastrophic and attritional assemblages. The general pattern of non-adult mortality, the value of certain demographic indices and the pattern of skeletal lesions in the mass graves investigated align more closely with the reference data for plague mortality, making their link with the Black Death the most likely scenario. Although a definite diagnosis will require further analysis, this study offers the first evidence of features that might distinguish between these two types of crisis, paving the way for future research on past epidemics and famines.
\end{abstract}


Keywords - mortality crisis, Black Death, famine, palaeodemography, historical demography, non-specific stress markers

\section{Introduction}

La fin du Moyen Âge a constitué en Europe une période de troubles, marquée par des crises de mortalité récurrentes, aux causes diverses, mais largement dominées par les épidémies et les épisodes de famine (Carpentier, 1962). Ces évènements, longtemps abordés sous l'unique angle de la recherche historique, ont depuis les années 1990 progressivement intégré le périmètre thématique des disciplines de l'archéologie. Depuis lors, un nombre croissant de travaux archéo-anthropologiques a été consacré aux témoins sépulcraux des crises de mortalité du passé, et notamment à leurs formes les plus évidentes : les sépultures multiples (structures funéraires ayant accueilli l'inhumation simultanée de plusieurs cadavres). Ces travaux se sont, pour certains, intéressés à documenter les caractéristiques d'ensembles funéraires dont on connaissait a priori la relation avec une crise de mortalité spécifique, qu'elle soit de nature épidémique (p. ex. Bizot et al., 2005 ; Signoli et al., 2007 ; Grainger et al., 2008 ; Godde et al., 2020), frumentaire (p. ex. Geber et Murphy, 2012 ; Geber, 2015), ou relève d'autres causes - notamment des conflits armés (p. ex. Fiorato et al., 2000 ; Wescott et al., 2012 ; Loe et al., 2014). D'autres ont, à l'inverse, tenté d'établir par l'examen des données archéologiques et paléobiologiques la cause probable de l'inhumation conjointe de nombreux défunts en sépultures multiples, pour des sites dont le statut était inconnu (p. ex. Castex et Réveillas, 2007 ; Castex, 2008 ; Castex et al., 2011 ; Colet et al., 2014). Cette entreprise s'avère bien souvent ardue $\mathrm{du}$ fait que, lorsqu'elles causent des décès rapides justifiant l'adoption de modes d'inhumation atypiques archéologiquement identifiables, épidémies et famines n'entraînent aucune lésion squelettique qui en permette le diagnostic rétrospectif. Exclusion faite des cas où les décès peuvent être rattachés à une épisode belliqueux au vu de la présence d'atteintes traumatiques péri-mortem ou à l'action d'un agent pathogène donné par le prisme d'analyses paléomicrobiologiques (p. ex. Drancourt et al., 1998 ; Grumbkow et al., 2011 ; Haensch et al., 2011 ; Spyrou et al., 2019), les anthropologues sont donc contraints de fonder leur réflexion sur les analogies que présentent leurs données avec celles issues de travaux de démographie historique (p. ex. Mallet, 1835 ; Hollingsworth et Hollingsworth, 1971 ; Dupâquier, 1979 ; Séguy et al., 2006) ou d'études d'ensembles funéraires en lien avec des crises dont la nature est connue. Ces données de référence sont aujourd'hui relativement nombreuses en ce qui concerne les épidémies, et notamment la peste dont on a pu révéler certaines particularités en termes de recrutement funéraire par âge et par sexe (p. ex. Castex, 2008 ; Tzortzis, 2009 ; Castex et Kacki, 2016) et de profil paléopathologique des victimes (p. ex. Kacki, 2017 ; 2020). D'autres travaux, moins nombreux, ont également permis de documenter les caractéristiques paléobiologiques des victimes de certains épisodes de famine (p. ex. Geber, 2014 ; 2015 ; Yaussy et al., 2016 ; DeWitte et Yaussy, 2020).

S'inscrivant en droite ligne des recherches susmentionnées, le présent article livre les résultats de l'étude de trois sépultures multiples du XIV ${ }^{\mathrm{e}}$ siècle, issues d'un ensemble funéraire attenant à la chapelle de Tous-les-Saints de Kutná Hora - Sedlec (République tchèque). Au travers de l'étude paléobiologique comparative de l'échantillon de squelettes exhumés de ces sépultures et d'une série ostéo-archéologique sub-contemporaine provenant d'un autre cimetière de la ville, il vise à discuter la nature de l'évènement responsable de ces inhumations de masse. Au regard des sources historiques disponibles concernant les crises que la région a connues à la fin du Moyen Âge (cf. infra), le propos se concentrera plus particulièrement sur les hypothèses d'une épidémie de peste ou d'un épisode de famine, et sur les indices permettant un diagnostic différentiel entre ces deux causes de surmortalité. À notre connaissance, aucune étude publiée n'a jusqu'alors embrassé une telle approche anthropobiologique pour tenter de différencier peste et famine dans le registre archéologique, les rares travaux sur le sujet s'étant cantonnés à l'exploitation des seules données chrono-stratigraphiques (p. ex. Lütgert, 2000).

\section{Matériel et méthodes}

\section{Les sépultures multiples de Kutná Hora - Sedlec}

Le site archéologique de la chapelle de Tous-les-Saints de Kutná Hora - Sedlec (dans la suite du texte "site de Sedlec") a fait l'objet de diverses campagnes de fouilles entre 2010 et 2018, dans le cadre de travaux de restauration de sa célèbre chapelle ossuaire. L'espace attenant à cet édifice a livré, outre de nombreuses sépultures individuelles, un total de 32 sépultures multiples, toutes attribuables avec certitude au XIV ${ }^{\mathrm{e}}$ siècle, mais se superposant pour certaines sur deux niveaux (Frolík, 2017 ; Brzobohata et al., 2019). Le nombre et la configuration de ces structures funéraires laissent envisager qu'elles sont en relation soit avec une crise de mortalité de longue durée, soit avec plusieurs crises de mortalité successives.

Selon les sources historiques, deux grands événements meurtriers pourraient être responsables des décès multiples à l'origine de ces inhumations de masse. Le premier correspond à un épisode de famine qui frappa la région au cours de l'année 1317, en corollaire d'une série de catastrophes (épizootie, inondations, guerre civile et hiver rude) (Lucas, 1930 ; Brazdil et al., 2018). Le second correspond à l'épidémie de Peste noire qui ravagea l'Europe au milieu du $\mathrm{XIV}^{\mathrm{e}}$ siècle et serait parvenue en Bohème depuis l'Italie au cours de l'année 1349 (Mengel, 2011). Certaines sépultures peuvent être rattachées avec une relative certitude à l'épisode de peste grâce à la découverte en leur sein de monnaies frappées en 1346 (Brzobohata et al., 2019). D'autres, et notamment celles auxquelles se superposent les tombes contenant ces monnaies, ne peuvent au contraire pas être attribuées avec certitude à l'un ou l'autre des évènements 
sus-cités en l'absence d'éléments datants. Ce sont ces dernières qui font l'objet de la présente étude, l'objectif étant d'évaluer si la première phase de sépultures multiples est également imputable à la Peste noire, l'épidémie pouvant avoir duré plusieurs mois, voire s'être prolongée sur plusieurs années, ou si elle relève d'un épisode antérieur de surmortalité (possiblement la famine de l'année 1317).

\section{Corpus et données de comparaison}

Le matériel étudié se compose en premier lieu de 68 squelettes exhumés de trois sépultures multiples du site de Sedlec, toutes attribuées à la première phase d'inhumations simultanées (tableau 1). De manière à caractériser les spécificités de cet échantillon par rapport au contexte de mortalité naturelle dans la population médiévale de Kutná Hora, un second échantillon, composé de 284 squelettes des $\mathrm{XII}^{\mathrm{e}}$ $\mathrm{XV}$ siècles provenant du cimetière attenant à la Cathédrale de Sedlec (dans la suite du texte "site de la Cathédrale"), a également été étudié.

\begin{tabular}{|l|c|c|c|c|c|}
\hline \multirow{2}{*}{$\begin{array}{c}\text { Groupe } \\
\text { d'âge }\end{array}$} & \multicolumn{4}{|c|}{ Sedlec } & \multirow{2}{*}{ Cathédrale } \\
\cline { 2 - 5 } & $\mathrm{Sp} \mathrm{765}$ & $\mathrm{Sp} 853$ & $\mathrm{Sp} 1578$ & Total & \\
\hline Immatures & 10 & 23 & 13 & 36 & 58 \\
\hline Adultes & 18 & 6 & 8 & 32 & 226 \\
\hline Total & $\mathbf{2 8}$ & $\mathbf{2 9}$ & $\mathbf{2 1}$ & $\mathbf{6 8}$ & $\mathbf{2 8 4}$ \\
\hline
\end{tabular}

Tableau 1. Effectif d'individus immatures et adultes dans les trois sépultures multiples du site de Sedlec et dans la série de la Cathédrale / Number of non-adults and adults in the three mass graves from the Sedlec burial site and in the skeletal assemblage from the Cathedral cemetery
Afin d'évaluer les éventuelles similitudes entre les caractéristiques paléobiologiques de l'échantillon de Sedlec et les spécificités des crises de mortalité documentées, des comparaisons ont été réalisées avec un large éventail de données se rapportant à des contextes d'épidémies de peste et de famines (tableau 2). Les premiers incluent des données paléobiologiques acquises par l'étude de plusieurs sites funéraires européens en relation avec la Peste noire ou ses résurgences, ainsi que des informations issues de registres de décès constitués lors d'épidémies de peste. Les seconds incluent les données récoltées pour deux sites archéologiques de l'archipel britannique en lien avec des épisodes de famine, ainsi que des données de démographie historique reflétant la mortalité lors d'années de famines en Irlande et en Finlande au XIX ${ }^{\mathrm{e}}$ siècle (voir Annexe 1).

\section{Méthodes de l'étude paléobiologique}

L'estimation de l'âge des sujets immatures s'est fondée en priorité sur le degré de minéralisation dentaire (Moorrees et al., 1963). Pour les individus dépourvus de restes dentaires ou dont les dents étaient totalement minéralisées, l'estimation a reposé sur le degré de maturation squelettique (Coqueugniot et al., 2010). Lorsqu'aucun de ces indicateurs n'était appréciable, elle s'est fondée sur les longueurs maximales des diaphyses des os longs (Stloukal et Hanáková, 1978). L'estimation de l'âge des adultes s'est limitée à distinguer les individus de moins de 30 ans sur la base des processus de maturation tardifs (Owings-Webb et Suchey, 1985). Leur sexe a été déterminé par l'application conjointe d'une méthode morphoscopique (Brůžek, 2002) et d'une méthode morphométrique probabiliste (Brůžek et al., 2017).

\begin{tabular}{|c|c|c|c|c|c|}
\hline Contexte & Dénomination & Datation & Effectif & Nature des données & Source \\
\hline \multirow{6}{*}{ Peste } & $\begin{array}{l}\text { East Smithfield, } \\
\text { Londres (Angleterre) }\end{array}$ & \multirow{4}{*}{ XIVe s. } & 485 & $\begin{array}{l}\text { Paléobiologie } \\
\text { (âge, sexe) }\end{array}$ & DeWitte, 2006 \\
\hline & $\begin{array}{l}\text { Hereford Cathedral, } \\
\text { Hereford (Angleterre) }\end{array}$ & & 185 & \multirow{3}{*}{$\begin{array}{c}\text { Paléobiologie } \\
\text { (âge, sexe, indicateurs } \\
\text { de stress) }\end{array}$} & \multirow{3}{*}{ Kacki, 2016} \\
\hline & $\begin{array}{l}\text { Les Fédons, } \\
\text { Lambesc (France) }\end{array}$ & & 133 & & \\
\hline & \begin{tabular}{|l|} 
Maria Troon, \\
Termonde (Belgique) \\
\end{tabular} & & 99 & & \\
\hline & $\begin{array}{l}\text { St. Botolph, } \\
\text { Londres (Angleterre) }\end{array}$ & 1603 & 1422 & \multirow{2}{*}{$\begin{array}{l}\text { Démographie } \\
\text { historique }\end{array}$} & Hollingsworth et Hollingsworth, 1971 \\
\hline & Genève (Suisse) & XVII ${ }^{\mathrm{e}} \mathrm{s}$. & 1718 & & Mallet, 1835 \\
\hline \multirow{6}{*}{ Famine } & $\begin{array}{l}\text { St. Mary Spital, } \\
\text { Londres (Angleterre) }\end{array}$ & $X I^{e}-X V I^{e} s$ & 814 & \multirow{2}{*}{$\begin{array}{c}\text { Paléobiologie } \\
\text { (âge, sexe, indicateurs } \\
\text { de stress) }\end{array}$} & \begin{tabular}{|l|} 
Yaussy et al., 2016; \\
Yaussy et DeWitte, 2018
\end{tabular} \\
\hline & \begin{tabular}{|l} 
Kilkenny Union \\
Workhouse (Irlande)
\end{tabular} & $\mathrm{XIX}^{\mathrm{e}} \mathrm{s}$. & 970 & & $\begin{array}{l}\text { Geber, } 2015 ; \\
\text { Geber et Murphy, } 2012\end{array}$ \\
\hline & $\begin{array}{l}\text { Workhouses Grande } \\
\text { famine (Irlande) }\end{array}$ & $1841-1851$ & 271884 & \multirow{4}{*}{$\begin{array}{l}\text { Démographie } \\
\text { historique }\end{array}$} & Guinnane et Ó Gráda, 2002a \\
\hline & Grande famine (Irlande) & $1841-1851$ & 1312211 & & \\
\hline & \multirow{2}{*}{$\begin{array}{l}\text { Famine Finlandaise } \\
\text { (Finlande) }\end{array}$} & $1832-1833$ & ND & & Pitkänen, 2002 \\
\hline & & $1857-1858$ & ND & & Pitkänen, 2002 \\
\hline
\end{tabular}

Tableau 2. Sites archéologiques et jeux de données de démographie historique intégrés à l'étude comparative. ND : information non disponible dans les sources consultées / Plague-and famine-related burial sites and historical demography datasets used for comparative purposes. ND: no data available 
Les données récoltées ont été utilisées pour calculer le taux de masculinité dans la fraction adulte de l'échantillon et pour mesurer les quotients de mortalité au sein de chaque classe d'âge immature - [0], [1-4], [5-9], [10-14] et [15$19]$ ans. À cette fin, les sujets immatures n'ayant pu être attribués avec certitude à une classe d'âge parmi deux contiguës ont fait l'objet d'une ventilation selon le principe de minimalisation des anomalies (Sellier, 1995). Les quotients de mortalité, obtenus en divisant le nombre des décès au sein de la classe d'âge considérée par le nombre total des individus d'âge égal ou supérieur (pour plus de détail, voir Henry, 1980), ont été comparés aux données issues de tables-types de mortalité considérées comme représentatives de la mortalité des populations pré-jennériennes (Ledermann, 1969), en considérant comme a priori une espérance de vie à la naissance comprise entre 25 et 35 ans (suivant les recommandations de Sellier, 1996). Cette démarche méthodologique, qui a précédemment fait ses preuves pour identifier des spécificités de recrutement liées à une mortalité par épidémie (p. ex. Castex, 2008 ; Castex et Kacki, 2016), a pour avantage d'autoriser l'identification des seules anomalies irréductibles (non imputables à des erreurs de ventilation d'individus d'âge incertain). Elle permet en outre de s'affranchir de l'influence d'éventuels problèmes de préservation des vestiges squelettiques des plus jeunes individus (à la différence d'une approche fondée sur l'analyse des proportions d'individus par classes d'âge). Les données de ces tables-types ont également été utilisées pour évaluer l'adéquation entre la proportion d'adultes de moins de 30 ans dans les échantillons étudiés et celle classiquement attendue en contexte de mortalité naturelle. Une analyse plus fine de la composition par âge de la fraction adulte des échantillons, en dépit de l'intérêt intrinsèque qu'elle aurait pu revêtir (voir notamment Tzortzis, 2009), n'a pas pu être menée du fait que l'effectif d'individus disposant de restes crâniens était trop limité pour permettre leur ventilation en classes d'âge décennales suivant la méthode des vecteurs de probabilité (Séguy et Buchet, 2011).

Les principaux os longs (humérus, radius, ulna, fémur et tibia) des adultes ont été systématiquement mesurés à l'aide d'une planche ostéométrique (d'après Bräuer, 1988). Les mesures obtenues pour les sites de Sedlec et de la Cathédrale ont été comparées par le biais d'une analyse de la variance (ANOVA), afin d'évaluer si ces échantillons se distinguaient par leurs dimensions corporelles, lesquelles peuvent être influencées par l'action de facteurs sanitaires, nutritionnels et sociaux durant la croissance (Steckel, 1995 ; Silventoinen, 2003).

Trois types d'indicateurs de stress parmi les plus couramment étudiés en anthropologie ont fait l'objet d'une recherche et d'un enregistrement systématique. Les hypoplasies linéaires de l'émail dentaire ont été cotées suivant une échelle distinguant, outre leur éventuelle absence (stade A), les formes à faible expression (stade B) des formes nettement marquées (stade C) (Kacki, 2017). Seule a été prise en compte la denture permanente, afin d'autoriser les comparaisons de prévalences entre fractions immatures et adultes des échantillons. Le stade lésionnel le plus élevé à l'échelle de la denture a été retenu à l'échelle individuelle. La présence ou l'absence de cribra orbitalia a également été consignée. Il en a été de même pour les réactions périostées tibiales, pour lesquelles a également été enregistré le degré de remodelage (actif, mixte ou remodelé) de chaque lésion (Mensforth et al., 1978). Les comparaisons de prévalences lésionnelles entre les sites ont été réalisées à l'aide du test exact de Fisher, en retenant un seuil de $5 \%$ comme risque de première espèce.

L'ensemble des données paléobiologiques individuelles et des données métriques relatives aux échantillons de Sedlec et de la Cathédrale sont consultables dans les Annexes 2-3.

\section{Résultats}

\section{Comparaison entre les sites de Sedlec et de la Cathédrale}

\section{Recrutement par âge et par sexe}

Le profil de mortalité de l'échantillon ostéo-archéologique de Sedlec présente de nombreuses anomalies par rapport à un modèle de mortalité naturelle (figure 1). En premier lieu, il rend compte d'une nette sous-représentation des enfants de moins d'un an. Il partage cette caractéristique avec l'échantillon du site de la Cathédrale, qui ne renferme plus généralement que peu d'individus de moins de 5 ans. Pour le site de Sedlec, les quotients de mortalité dans les deux classes suivantes ([1-4] et [5-9] ans) sont compatibles avec les valeurs représentatives d'une mortalité naturelle, se plaçant toutefois respectivement au niveau de la limite basse

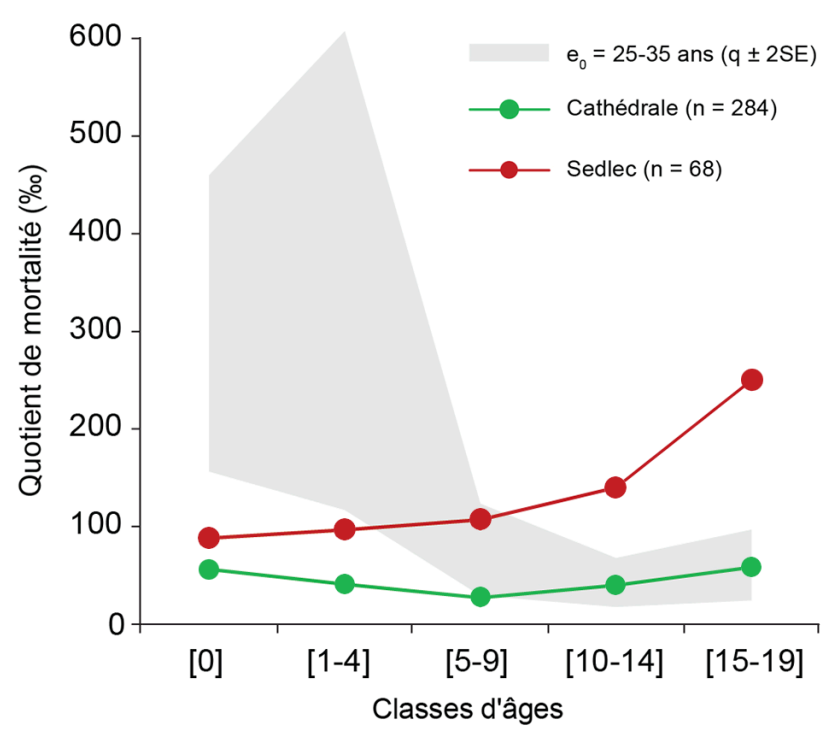

Figure 1. Profils de mortalité des échantillons des sites de Sedlec et la Cathédrale comparés à l'intervalle des quotients de mortalité théoriques en contexte de mortalité naturelle (d'après Ledermann, 1969) / Age-at-death distribution of non-adults from the Sedlec burial site and the Cathedral cemetery, compared with the range of theoretical values for non-epidemic mortality (after Ledermann, 1969) 
et de la limite haute de l'intervalle théorique. A contrario, les individus appartenant aux dernières classes d'âge immatures ([10-14] et [15-19] ans) sont fortement surreprésentés, tant en comparaison des valeurs théoriques que des quotients de mortalité relevés pour le site de la Cathédrale. Le profil de mortalité des sujets immatures des sépultures multiples de Sedlec se caractérise donc par une augmentation relativement constante des quotients de mortalité depuis la petite enfance jusqu'à la fin de l'adolescence, suivant une courbe inverse à celle représentant une mortalité naturelle.

La fraction adulte de l'échantillon de Sedlec renferme une proportion de sujets de moins de 30 ans relativement élevée ( $\mathrm{n}=7$, soit $22 \%)$. Cette proportion, supérieure à celle relevée pour le site de la Cathédrale (16\%), excède l'intervalle des valeurs moyennes représentatives d'un contexte de mortalité naturelle, qui oscillent entre $11,2 \%$ et $15,7 \%$ selon l'espérance de vie à la naissance considérée (d'après les données de Ledermann, 1969).

Chez les adultes du site de Sedlec dont le sexe a pu être déterminé $(\mathrm{n}=18)$, le taux de masculinité s'élève à $72 \%$ (figure 2). Cette forte surreprésentation masculine, qui tranche avec le sex ratio équilibré qui caractérise la plupart des populations naturelles, s'exprime également pour le site de la Cathédrale, et ce, en intensité semblable. Cette anomalie semble donc, plus que refléter une spécificité en lien avec la cause des décès, constituer une caractéristique de la population médiévale de Kutná Hora, dont les possibles raisons seront discutées plus loin.

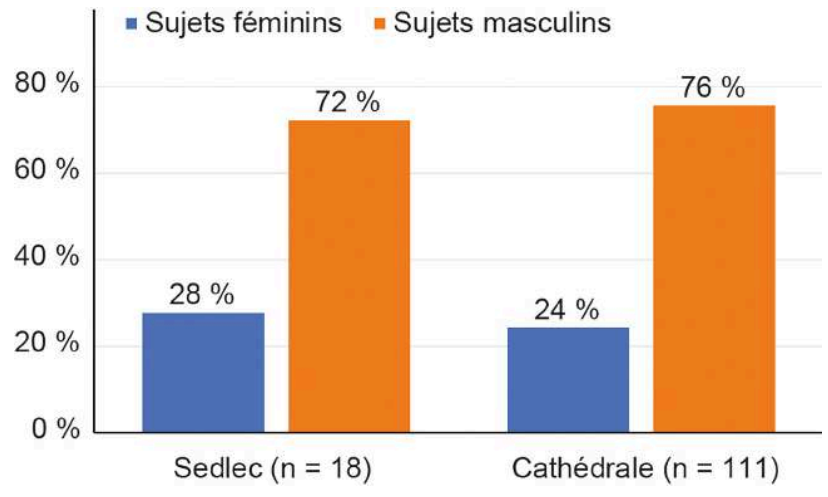

Figure 2. Distribution par sexe des individus adultes dans les échantillons des sites de Sedlec et de la Cathédrale / Sex distribution of adults in the skeletal assemblages from the Sedlec burial site and Cathedral cemetery

\section{Caractéristiques ostéométriques}

En conséquence du nombre limité d'individus féminins dans le corpus de Sedlec, les comparaisons entre longueurs des os longs dans les deux séries n'ont pu être réalisées que pour les sujets masculins. Les résultats obtenus indiquent une tendance à des dimensions osseuses moindres chez les hommes du site de Sedlec (figure 3). Les différences intersites sont valables quel que soit l'os considéré ; elles ne sont toutefois statistiquement significatives que pour l'humérus et le tibia.

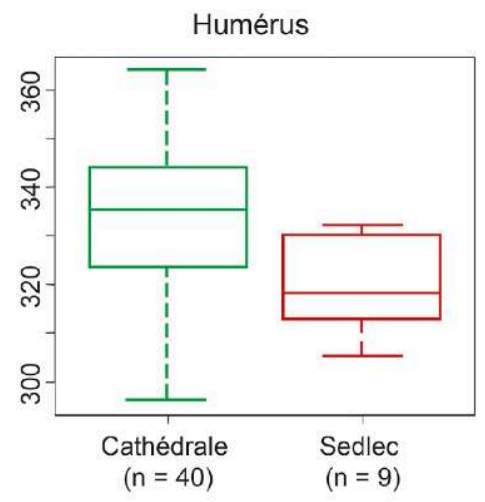

Fémur

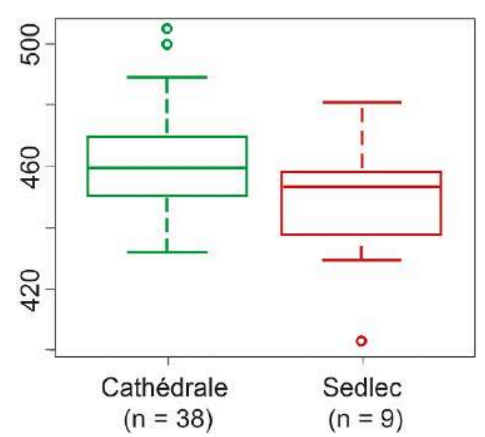

Radius

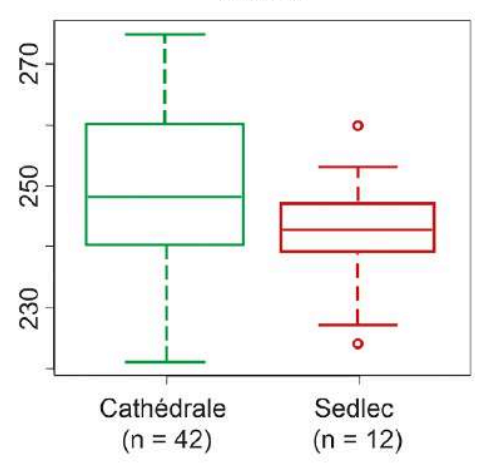

Tibia

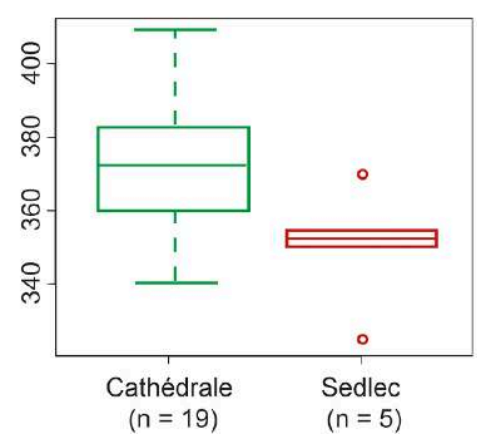

Ulna

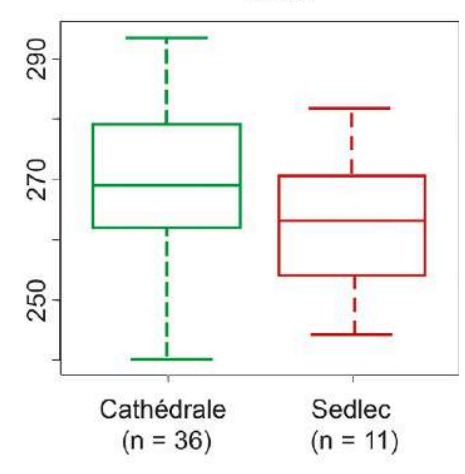

\begin{tabular}{ll}
\hline Os & p-valeur \\
\hline Humérus & $\mathbf{0 , 0 0 7}$ \\
Radius & 0,085 \\
Ulna & 0,114 \\
Fémur & 0,085 \\
Tibia & $\mathbf{0 , 0 1 7}$ \\
\hline
\end{tabular}

Figure 3. Dispersion des longueurs des os longs des sujets adultes masculins des sites de Sedlec et de la Cathédrale, accompagnée des résultats de l'ANOVA à un facteur comparant les deux sites / Boxplots showing dispersion of long bone length in the adult males from the Sedlec assemblage and Cathedral cemetery, with results of one-way ANOVA comparisons between the sites 


\section{Caractéristiques paléopathologiques}

Les hypoplasies linéaires de l'émail dentaire sont communes dans l'une et l'autre des collections, la proportion d'individus concernés y étant légèrement supérieure chez les adultes (figure 4). Elles sont toutefois significativement plus fréquentes chez les sujets du site de la Cathédrale $(p<0,001)$. Une différence est également perceptible en ce qui concerne les hypoplasies les plus marquées ( $\mathrm{p}=0,041)$ qui sont notamment présentes en fréquence plus de deux fois supérieure chez les sujets immatures du site de la Cathédrale.

Les cribra orbitalia sont significativement plus fréquentes dans l'échantillon de Sedlec (32\%) que dans celui du site de la Cathédrale $(14 \%)(p=0,039)$. Cependant, la différence entre échantillons est marquée par de fortes variations selon les classes d'âge (figure 5). Avant l'âge de 5 ans, soit la période au cours de laquelle se forme cet indicateur de stress (Stuart-Macadam, 1985), les porosités du toit orbitaire sont plus rares chez les sujets de Sedlec que chez ceux du site de la Cathédrale. La tendance s'inverse après 5 ans, les séquelles de ces lésions concernant une proportion beaucoup plus élevée de sujets de la série de Sedlec jusqu'avant l'âge de 20 ans. À l'âge adulte, la fréquence de cribra orbitalia décline, de même que l'écart de prévalence entre les deux sites.

Seuls trois individus du site de Sedlec présentent des réactions périostées tibiales (figure 6). Leur fréquence dans cette collection est significativement moindre que dans celle provenant du site de la Cathédrale $(\mathrm{p}=0,002)$. La différence relevée tient essentiellement à une proportion bien plus élevée de lésions remodelées chez les individus morts hors contexte de crise de mortalité.

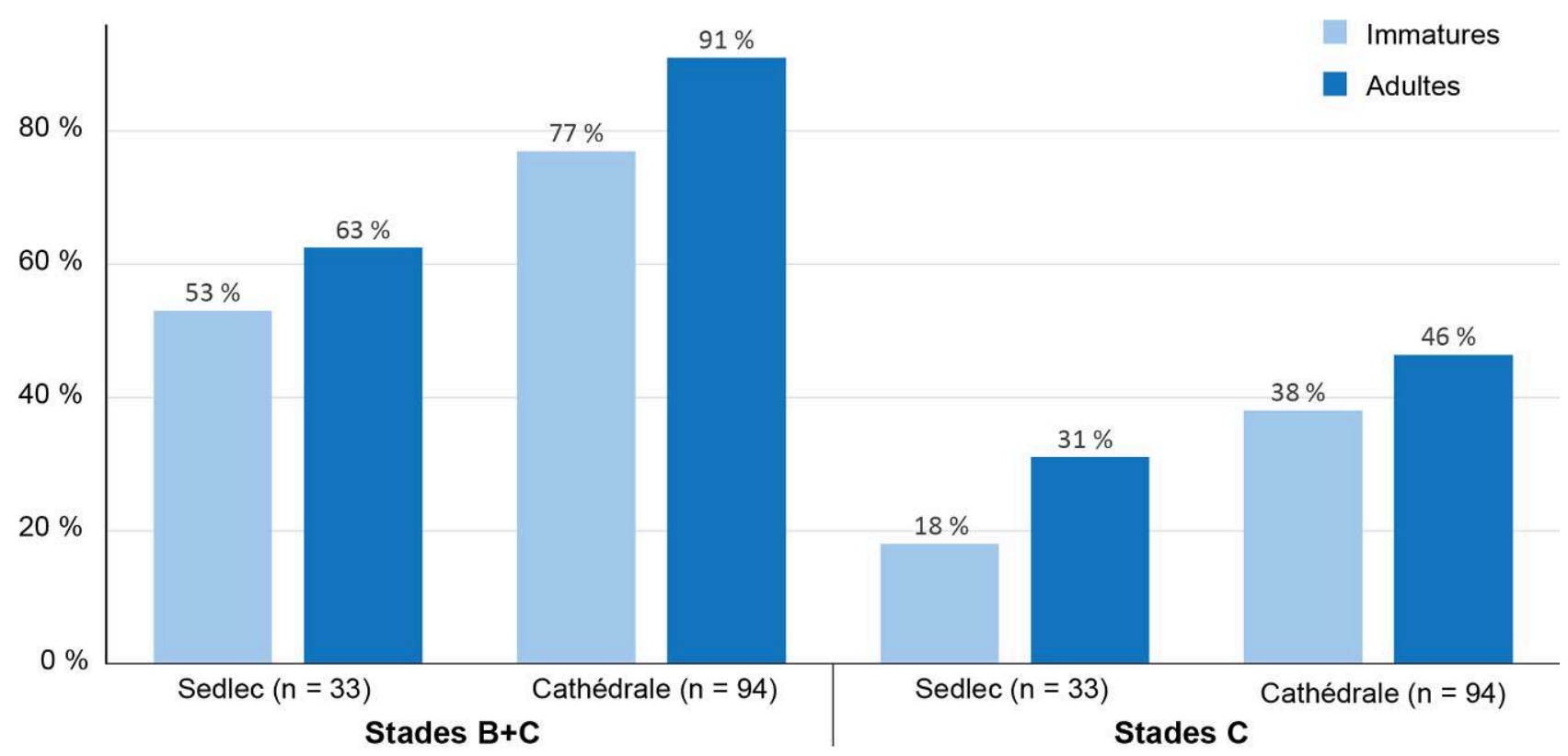

Figure 4. Prévalence des hypoplasies linéaires de l'émail par site, stade d'expression et catégorie d'âge / Prevalence of linear enamel hypoplasia by site, severity stage and age group

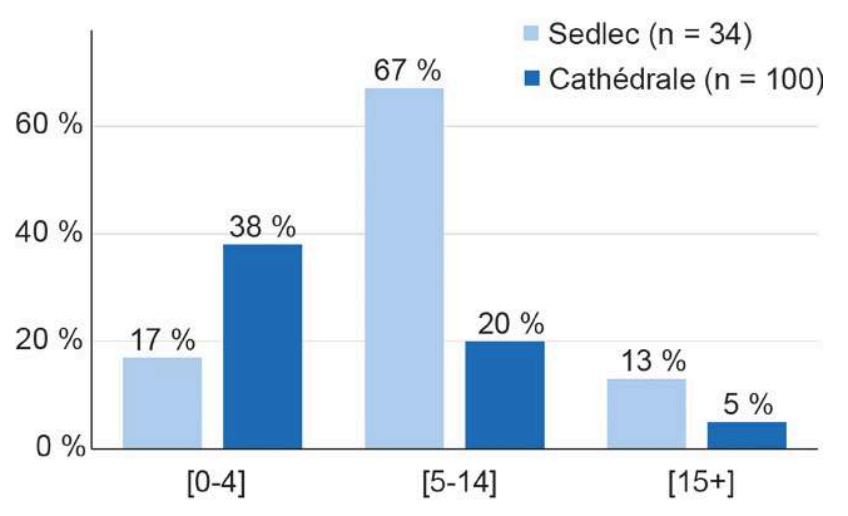

Figure 5. Prévalence des cribra orbitalia par site et catégorie d'âge / Prevalence of cribra orbitalia by site and age group

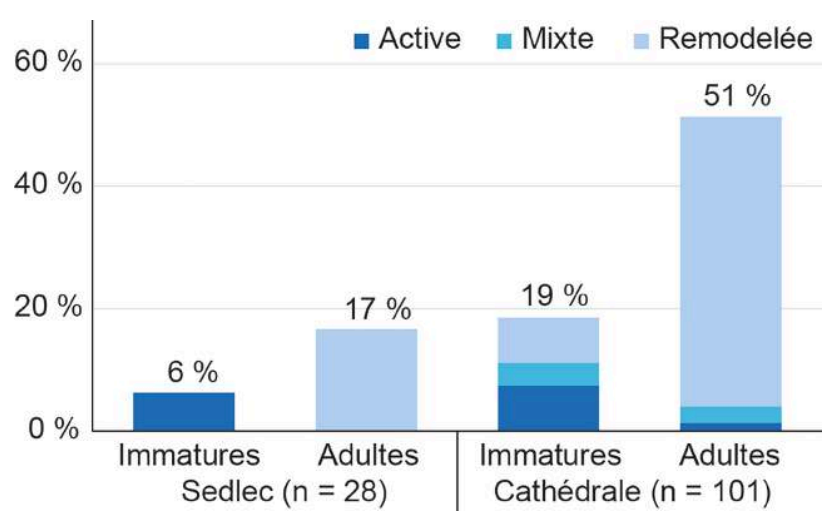

Figure 6. Prévalence des réactions périostées tibiales par site et catégories d'âge, selon leur degré de remodelage / Prevalence of tibial periostitis by site and age group, with distinction according to the stage of remodelling 


\section{Confrontation aux contextes d'épidémies de peste}

Le profil de mortalité de l'échantillon de Sedlec présente certaines similitudes avec ceux établis pour des contextes de peste (figure 7). À l'instar de ces derniers, il rend compte d'une sous-représentation des enfants de moins d'un an et d'une faible part de décès entre 1 et 4 ans, tandis que les quotients de mortalité y sont élevés chez les sujets immatures de plus de 10 ans. La seule différence notable concerne la classe des [5-9] ans, pour laquelle l'échantillon tchèque présente un quotient de mortalité inférieur à celui enregistré pour la plupart des contextes d'épidémies de peste documentés. Ce quotient est toutefois semblable à celui caractérisant le corpus archéologique en relation avec la Peste noire qui, plus généralement, présente un profil de mortalité très similaire à celui de l'échantillon de Sedlec. Son allure générale est également assez proche de celle du profil de mortalité enregistré au XVII ${ }^{\mathrm{e}}$ siècle à Genève en contexte de peste.
La confrontation s'avère plus délicate en ce qui concerne les indicateurs de stress, compte tenu de leur prévalence variable dans les séries de pestiférés (tableau 3). Au regard de la plage des valeurs relevées pour ces dernières, les hypoplasies de l'émail apparaissent relativement peu fréquentes dans l'échantillon étudié. Le ratio entre leur prévalence chez les sujets immatures et leur prévalence chez les adultes est proche de celui observé pour les sites d'Hereford et des Fédons. Les cribra orbitalia sont, pour leur part, relativement fréquentes dans l'échantillon de Sedlec, notamment chez les sujets immatures, qui sont près de quatre fois plus souvent concernés que les adultes. A contrario, la prévalence des réactions périostées tibiales est faible en comparaison de celles relevées dans les séries de pestiférés. Les adultes sont près de trois fois plus touchés que les sujets immatures, soit un différentiel comparable à celui constaté pour les sépultures de peste d'Hereford.
A.

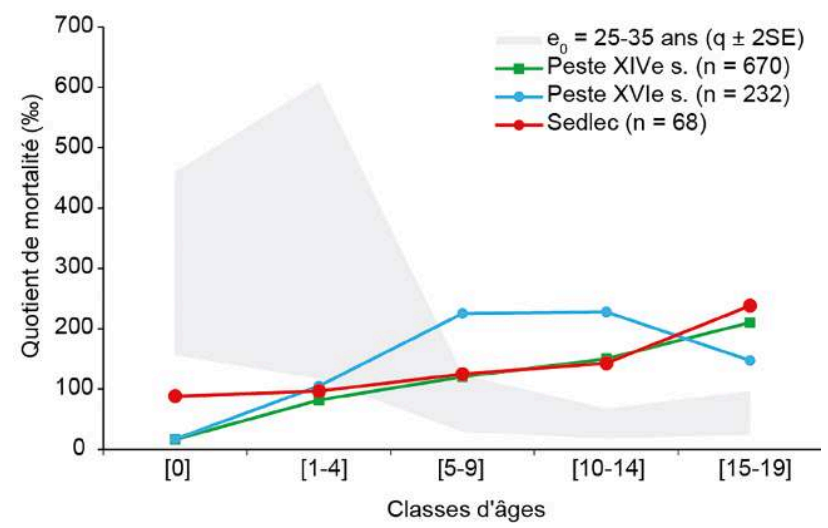

B.

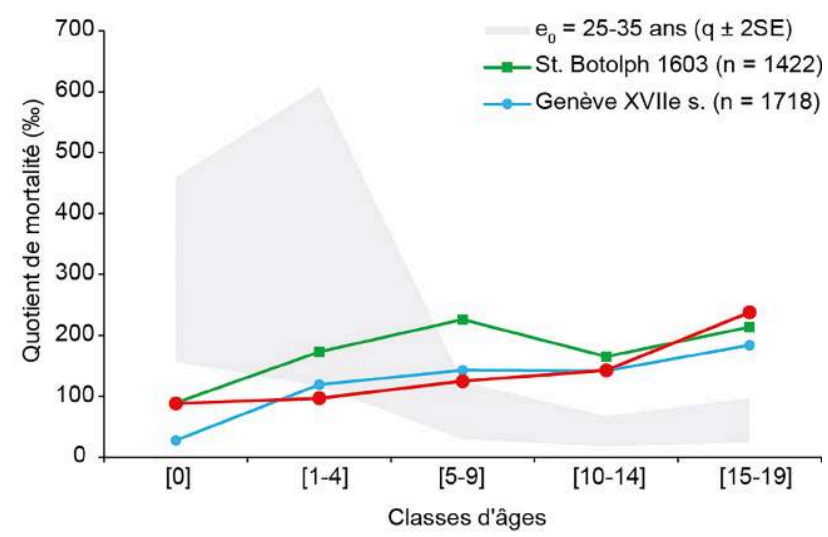

Figure 7. Confrontation du profil de mortalité de l'échantillon de Sedlec à ceux caractérisant des épidémies de peste documentées pour des cimetières archéologiques (A) et par des données de démographie historique (B). L'intervalle des valeurs théoriques pour un contexte de mortalité naturelle (d'après Ledermann, 1969) est fourni à titre de comparaison / Age-at-death distribution of non-adults from the Sedlec skeletal assemblage, compared with mortality profiles of plague victims inferred from the study of burial sites (A) and historical demography (B). The range of theoretical values for non-epidemic mortality (after Ledermann, 1969) is provided for comparison

\begin{tabular}{|l||c|c|c|}
\hline \multicolumn{1}{|c|}{ Site } & \multicolumn{3}{|c|}{ HLED } \\
\cline { 2 - 4 } & $\begin{array}{c}\text { IM } \\
(\%)\end{array}$ & $\begin{array}{c}\text { AD } \\
(\%)\end{array}$ & Ratio \\
\hline Sedlec & 18 & 31 & 0,68 \\
\hline Hereford & 48 & 68 & 0,71 \\
\hline Les Fédons & 38 & 55 & 0,69 \\
\hline Termonde & 23 & 15 & 1,53 \\
\hline Kilkenny & 17 & 21 & 0,81 \\
\hline St. Mary Spital & 55 & 36 & 1,53 \\
\hline
\end{tabular}

\begin{tabular}{|c|c|c|}
\hline \multicolumn{3}{|c|}{ Cribra orbitalia } \\
\hline $\begin{array}{c}\text { IM } \\
(\%)\end{array}$ & $\begin{array}{c}\text { AD } \\
(\%)\end{array}$ & Ratio \\
\hline 50 & 13 & 3,85 \\
\hline 42 & 13 & 3,23 \\
\hline 43 & 20 & 2,15 \\
\hline 52 & 36 & 1,44 \\
\hline 25 & 13 & 1,92 \\
\hline 48 & 37 & 1,3 \\
\hline
\end{tabular}

\begin{tabular}{|c|c|c|}
\hline \multicolumn{3}{|c|}{ Réactions périostées } \\
\hline $\begin{array}{c}\text { IM } \\
(\%)\end{array}$ & $\begin{array}{c}\text { AD } \\
(\%)\end{array}$ & Ratio \\
\hline 6 & 17 & 0,35 \\
\hline 15 & 41 & 0,37 \\
\hline- & - & - \\
\hline 43 & 33 & 1,3 \\
\hline 13 & - & - \\
\hline 8 & 25 & 0,32 \\
\hline
\end{tabular}

\begin{tabular}{|l|}
\hline Source \\
\hline Cette étude \\
\hline Kacki, 2016 \\
\hline Kacki, 2016 \\
\hline Kacki, 2016 \\
\hline Geber, 2015 ; Geber et Murphy, 2012 \\
\hline Yaussy et al., 2016; Yaussy et DeWitte, 2018 \\
\hline
\end{tabular}

Tableau 3. Confrontation des prévalences des indicateurs de stress entre l'échantillon de Sedlec et différentes séries de pestiférés et de victimes de la famine. HLED : hypoplasies linéaires de l'émail dentaire. Seules les hypoplasies marquées sont prises en compte dans la comparaison / Comparison of the prevalence of non-specific stress indicators between the Sedlec skeletal assemblage and skeletal series from various plague- or famine-related burial sites. HLED: linear enamel hypoplasia. Only well-developed dental defects were included in the comparison 


\section{Confrontation aux contextes de famine}

Les quotients de mortalité obtenus pour l'échantillon de Sedlec sont, pour certaines classes d'âge, compatibles avec ceux relevés en temps de famine (figure 8). C'est notamment le cas pour les sujets de moins d'un an, qui ne comptent que pour une faible part des décès enregistrés dans ces contextes. La mortalité dans la classe d'âge des [5-9] ans est également conforme aux valeurs attendues lors d'épisodes de famine, avoisinant les valeurs basses enregistrées pour la crise irlandaise, les valeurs hautes relevées lors de deux famines du XIX ${ }^{\mathrm{e}}$ siècle en Finlande. Le quotient de mortalité élevé chez les [10-14] ans du corpus tchèque est équivalent à ce que l'on peut observer pour les maisons de travail (workhouses) lors de la Grande famine irlandaise (dont Kilkenny représente un témoin archéologique). Toutefois, deux différences sont perceptibles : les sujets d'âge compris entre 1 et 4 ans sont largement moins représentés à Sedlec qu'en temps de famine ; à l'inverse, les grands adolescents y sont en très large excédent. Le profil de Sedlec présente en conséquence une allure générale divergente de tous ceux constituant le corpus de donnés de comparaison.

Les prévalences d'indicateurs de stress dans l'échantillon de Sedlec ne se distinguent pas de manière notable de celles enregistrées pour les sépultures de victimes de la famine des sites de Kilkenny et de St. Mary Spital (tableau 3). Certaines différences apparaissent toutefois lorsque l'on s'intéresse aux ratios entre proportions d'individus immatures et adultes présentant ces changements pathologiques. Pour les hypoplasies, ce rapport se révèle plus faible à Sedlec qu'en contexte de famine. Pour les cribra orbitalia, il est au contraire bien plus élevé, témoignant ainsi d'un différentiel de prévalence entre sujets de moins et plus de 20 ans au moins deux fois plus important pour le site de Sedlec. Les réactions périostées s'observent au contraire dans des proportions proches de celles rapportées pour le site de St. Mary Spital.

\section{Discussion}

Les trois sépultures multiples de Kutná Hora - Sedlec étudiées n'ont pu, sur la seule base des informations stratigraphiques recueillies lors de la fouille, être attribuées à l'une ou l'autre des crises de mortalité rapportées dans les sources historiques pour la première moitié du XIV ${ }^{e}$ siècle. Si l'antériorité de ces sépultures à certaines tombes datées avec certitude des années de la Peste noire est avérée, rien ne permet en effet de préciser la durée de l'intervalle chronologique qui les sépare, qui pourrait aussi bien être de plusieurs décennies (lien potentiel avec la famine de 1317) que de seulement quelques mois (début de l'épidémie de Peste noire). L'étude paléobiologique des squelettes exhumés apporte, à cet égard, certains éléments d'importance pour orienter le diagnostic rétrospectif. Elle révèle diverses particularités de l'échantillon ostéo-archéologique constitué (déséquilibre du sex ratio, anomalies du profil de mortalité par âge avant 20 ans, divergence de profil lésionnel par rapport à un contexte de mortalité naturelle), dont certaines trouvent des parallèles dans un type de crise de mortalité plutôt que l'autre.

Le fort déséquilibre du sex ratio au profit des sujets masculins pourrait, à première vue, être considéré comme un argument en faveur de l'hypothèse d'un contexte de disette. En effet, tandis que la peste entraîne généralement une mortalité équilibrée entre les deux sexes (Castex et Kacki, 2016), voire légèrement plus élevée chez les femmes (Signoli et al., 2002), de nombreux auteurs ont avancé que la famine causerait plus de décès chez les hommes (p. ex. MacIntyre, 2002 ; Ó Gráda, 2009), du fait que les femmes possèdent davantage de masse graisseuse sous-cutanée et jouiraient de défenses immunitaires améliorées par les œstrogènes, ce qui leur octroierait de meilleures chances de survie face aux maladies infectieuses opportunistes se développant dans un tel contexte. Cette hypothèse ne se
A.

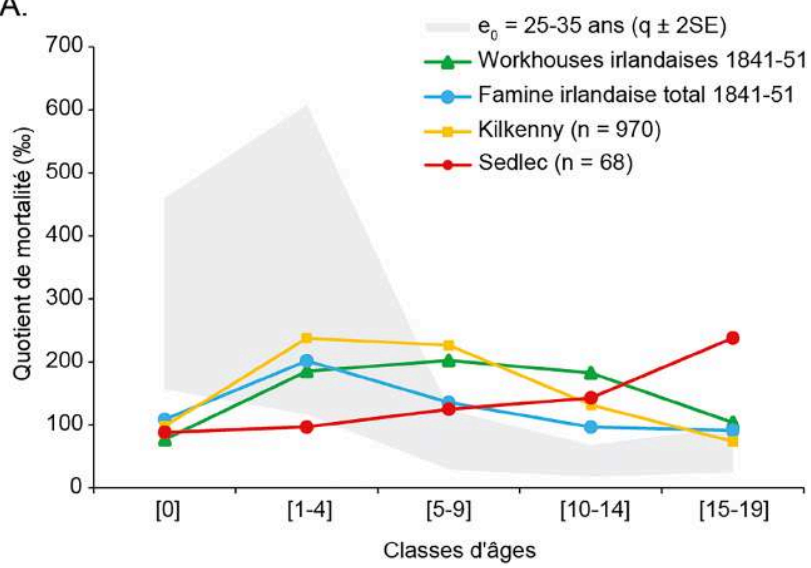

B.

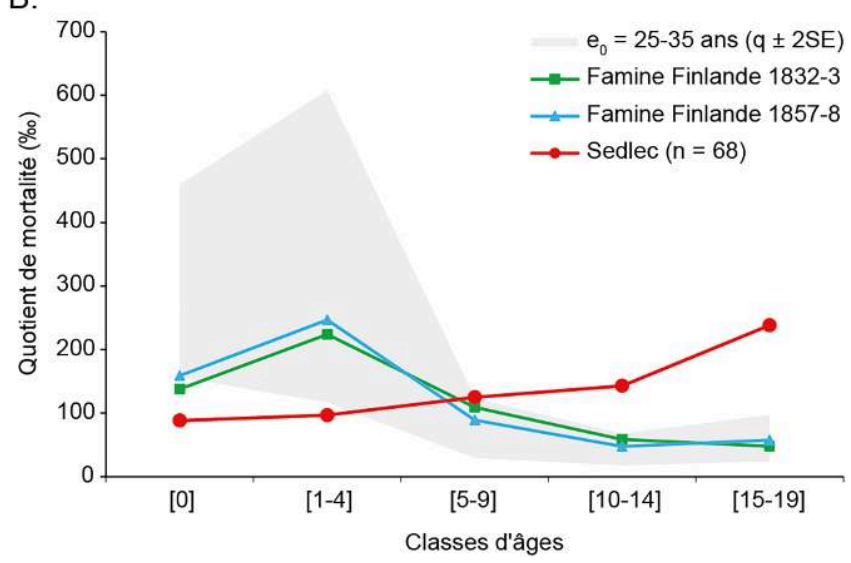

Figure 8. Confrontation du profil de mortalité de l'échantillon de Sedlec à ceux restitués pour la Grande famine irlandaise (A) et pour deux crises frumentaires du XIXe siècle en Finlande (B). L'intervalle des valeurs théoriques pour un contexte de mortalité naturelle (d'après Ledermann, 1969) est fourni à titre de comparaison / Age-at-death distribution of non-adults from the Sedlec skeletal assemblage, compared with mortality profiles for the Great Irish Famine (A) and for two $19^{\text {th }}$ century famines in Finland (B). The range of theoretical values for non-epidemic mortality (after Ledermann, 1969) is provided for comparison 
vérifie toutefois pas en ce qui concerne les individus adultes exhumés d'ensembles sépulcraux archéologiques en lien avec des crises frumentaires (Jones et Walker, 2012 ; Geber, 2015 ; DeWitte et Yaussy, 2020), ni dans certaines études de démographie historique ayant documenté la mortalité en temps de famine (Guinnane et Ó Gráda, 2002b ; Pitkänen, 2002). L'identification d'une surreprésentation masculine semblable pour le site de la Cathédrale semble par ailleurs exclure que cette anomalie soit le fait principal du contexte de mortalité et suggère qu'il s'agirait plutôt d'une caractéristique de la population médiévale de Kutná Hora. Elle s'explique probablement du fait que la ville connut son apogée grâce à ses mines d'argent, dont l'exploitation à partir du XIII ${ }^{\mathrm{e}}$ siècle entraina en sa toute fin et au siècle suivant une vague migratoire de jeunes hommes, venus essentiellement d'Allemagne, pour y travailler (Blanchard, 2005). Cette surreprésentation masculine, documentée par ailleurs pour d'autres sites miniers comme celui de Hallstatt (taux de masculinité de $62 \%$ ) (Pany, 2003), ne peut donc pas être considérée comme un bon argument dans la discussion sur la nature de la crise de mortalité.

L'examen du profil de mortalité avant 20 ans apporte davantage d'informations. Il met en évidence des anomalies patentes dans la composition par âge de l'échantillon non adulte en comparaison d'un modèle de mortalité naturelle, certaines classes d'âge accusant un déficit tandis que d'autres se caractérisent par une surreprésentation. Prises individuellement, la plupart de ces anomalies ne permettent toutefois pas de distinguer un épisode de peste d'une crise alimentaire, du fait que les quotients de mortalité qui s'y rapportent présentent une variabilité non négligeable selon les contextes - p. ex. différence de quotients de mortalité des [5-14] ans entre les séries de pestiférés du XIV et celles du XVI ${ }^{\mathrm{e}}$ siècle (Castex et Kacki, 2016 ; Kacki, 2017), de même qu'entre la population générale et la population pauvre des maisons de travail lors de la Grande famine irlandaise (Guinnane et Ó Gráda, 2002a). Ainsi, la sous-représentation des moins de un an relevée à Sedlec ne renseigne aucunement sur la cause potentielle des décès, s'observant indifféremment pour les deux types de crises considérées. Il en va de même des quotients de mortalité qu'enregistrent les classes d'âge [5-9] et [10-14], qui trouvent dans les données de comparaison des équivalents tant chez les victimes de la peste que chez celles de la famine. La surreprésentation des grands adolescents, fréquente en contexte d'infection pesteuse, est également rapportée, certes à de plus bas niveaux, pour certaines crises frumentaires. Aussi, plus que les quotients de mortalité, c'est la représentation respective des sujets des différentes classes d'âge immatures qui permet la différenciation entre ces deux types de crises. En rend compte l'allure des courbes de mortalité, qui permet de rapprocher le site de Sedlec de certains référentiels relatifs à des épidémies de peste, tandis que l'accroissement continu des quotients de mortalité qui s'y observe depuis la petite enfance jusqu'à la fin de l'adolescence ne trouve aucun analogue parmi les profils de mortalité se rapportant à la famine.

Plus informatifs encore sont certains indices démographiques, qui permettent d'objectiver ces différences (tableau 4). Ainsi, la proportion de sujets immatures morts entre 1 et 4 ans est, dans le jeu de données de comparaison, toujours inférieure à $30 \%$ pour les contextes d'épidémies de peste, tandis qu'elle égale ou excède cette valeur dans les contextes de famine. De même, la part des non adultes morts entre 15 à 19 ans ne dépasse jamais $10 \%$ chez les victimes de la famine, alors qu'elle n'est jamais inférieure à cette limite chez les victimes de la peste. Plus parlant encore est le ratio entre individus de moins et de plus de 10 ans, qui est toujours inférieur à 2,5 dans les contextes de peste alors qu'il est toujours supérieur à cette valeur dans les contextes de famine. Les valeurs de ces différents indices pour l'échantillon ostéo-archéologique de Sedlec accréditent l'hypothèse d'un lien des sépultures multiples étudiées avec une épidémie de peste.

\begin{tabular}{|l|l|c|c|c|l|}
\hline Contexte & Site ou localité & $\mathbf{D}_{\mathbf{1 - 4}} / \mathbf{D}_{\mathbf{I M}}$ & $\mathbf{D}_{\mathbf{1 5 - 1 9}} / \mathbf{D}_{\mathbf{I M}}$ & $\mathbf{D}_{\mathbf{0 - 9}} / \mathbf{D}_{\mathbf{1 0 - 1 9}}$ & Source des données \\
\hline \multirow{4}{*}{ Indéterminé } & Sedlec & 0,18 & 0,29 & 1,12 & Cette étude \\
\hline \multirow{5}{*}{ Peste } & East Smithfield & 0,13 & 0,37 & 0,54 & DeWitte, 2006 \\
\cline { 2 - 6 } & Hereford & 0,29 & 0,11 & 2,43 & Kacki, 2016 \\
\cline { 2 - 6 } & Les Fédons & 0,26 & 0,10 & 1,81 & Kacki, 2016 \\
\cline { 2 - 6 } & Termonde & 0,09 & 0,20 & 0,96 & Kacki, 2016 \\
\cline { 2 - 6 } & St. Botolph* & 0,26 & 0,17 & 2,08 & Hollingsworth et Hollingsworth, 1971 \\
\cline { 2 - 6 } & Genève* & 0,24 & 0,24 & 1,21 & Mallet, 1835 \\
\hline \multirow{5}{*}{ Famine } & Kilkenny & 0,37 & 0,06 & 4,50 & Geber, 2015 \\
\cline { 2 - 6 } & Irlande workhouses* & 0,31 & 0,09 & 2,50 & Guinnane et Ó Gráda, 2002a \\
\cline { 2 - 6 } & Irlande total* & 0,36 & 0,10 & 3,50 & Guinnane et Ó Gráda, 2002a \\
\cline { 2 - 6 } & Finlande 1832-1833* & 0,30 & 0,06 & 6,53 & Pitkänen, 2002 \\
\cline { 2 - 6 } & Finlande 1957-1958* & 0,43 & 0,07 & 7,15 & Pitkänen, 2002 \\
\hline
\end{tabular}

* Données de démographie historique / data from historical demography.

Tableau 4. Divers indices démographiques dans l'échantillon de Sedlec, comparés à leurs valeurs en contexte de peste ou de famine (données issues de travaux anthropologiques et de démographie historique) / Demographic indices in the Sedlec skeletal assemblage, compared with their values in times of plague or famine (data from anthropological and historical demography studies) 
L'interprétation s'avère plus délicate en ce qui concerne les résultats de l'analyse des indicateurs de stress. La simple confrontation de leurs prévalences dans l'échantillon de Sedlec avec celles enregistrées pour diverses séries de pestiférés et de victimes de la famine ne permet pas de trancher en faveur d'un contexte de mortalité plus qu'un autre. En est pour cause la forte variabilité de fréquences de ces stigmates d'un site à l'autre, qui tient probablement tant à l'influence de facteurs socio-économiques et environnementaux dans leur survenue (Ó Gráda, 2009 ; Geber, 2015) qu'à des divergences méthodologiques dans les protocoles présidant à leur cotation et au calcul de leurs prévalences dans différentes études. En ce sens, le recours au ratio entre leurs fréquences respectives chez les sujets immatures et les adultes s'avère plus pertinent, permettant de s'affranchir a minima du second écueil. Ce rapport, s'il s'avère peu informatif en ce qui concerne les réactions périostées, tend, pour les hypoplasies de l'émail et les cribra orbitalia, à rapprocher l'échantillon de Sedlec de certaines séries de pestiférés (tableau 3). Cette proximité est renforcée, en ce qui concerne les hypoplasies, par la comparaison menée avec le site de la Cathédrale, qui révèle la moindre fréquence de ces stigmates chez les individus morts lors de l'épisode de crise auquel se rattachent les sépultures multiples étudiées. Cette même caractéristique a précédemment été rapportée pour les individus immatures de séries de pestiférés, qui présentaient moins d'hypoplasies sévères (stade C) que leurs homologues morts hors contexte épidémique (Kacki, 2016). Au contraire, des travaux portant sur des sépultures de victimes de la famine ont conclu à une fréquence significativement plus importante d'hypoplasies chez ceux-ci que chez les individus morts hors temps de crise (Yaussy et DeWitte, 2018).

Un dernier résultat méritant discussion est l'écart de dimensions des os longs entre les hommes de l'échantillon de Sedlec et ceux du site de la Cathédrale. Si les différences relevées ne sont statistiquement démontrées que pour l'humérus et le tibia, tous présentent des longueurs moyennes plus faibles chez les individus du premier site. Ce pattern général pourrait indiquer que ces sujets ont éprouvé des conditions nutritionnelles plus défavorables durant leur croissance. De précédentes études ont notamment montré que les adultes ayant connu un épisode de disette au cours de leur enfance présentent des statures réduites en comparaison de leurs prédécesseurs ou leurs successeurs (Kozlov et Samsonova, 2005 ; Ó Gráda, 2009). À cet égard, il est intéressant de noter que l'épidémie de Peste noire a frappé la Bohème quelque 30 ans après la famine de 1317 (Lucas, 1930 ; Brazdil et al., 2018). Les caractéristiques ostéométriques de la série de Sedlec pourraient ainsi indiquer qu'une partie des individus exhumés des sépultures multiples seraient morts de la peste en 1349, après avoir subi la famine de 1317 durant leur enfance. Cette hypothèse doit cependant être considérée avec prudence. D'une part, on ne peut pas exclure que des individus ayant subi durant leur enfance des privations nutritionnelles ou d'autres conditions délétères suffisamment graves pour entraver leur croissance auraient, par la suite, eu un risque accru de mourir dans des conditions de famine (DeWitte et Yaussy, 2017); on notera cependant que les adultes victimes de la Grande famine irlandaise exhumés du site de Kilkenny présentaient une stature moyenne comparable à celles des individus subcontemporains décédés hors contexte de disette (Geber, 2015). D'autre part, il est possible que les écarts enregistrés ne soient pas en lien avec la nature des décès mais soient imputables aux vagues migratoires précédemment mentionnées, dont l'influence pourrait être plus forte pour les sépultures multiples du XIV ${ }^{\mathrm{e}}$ siècle de Sedlec que pour l'échantillon de la Cathédrale, constitué pour partie d'individus morts avant ces apports allochtones. Il est également envisageable que ces différences trouvent leur origine dans la conjonction entre ce facteur migratoire et le contexte de crise, une récente étude ayant démontré qu'au sein d'une même localité la mortalité lors de certaines épidémies de peste fut plus élevée chez les individus s'y étant récemment installés (Galanaud et al., 2020).

Pris dans leur ensemble, les résultats obtenus ne fournissent in fine aucun indice permettant de suspecter le lien des sépultures multiples étudiées avec un épisode de famine. Certains d'entre eux, notamment ceux concernant la composition par âge de la fraction immature de l'échantillon, tendent au contraire à étayer l'hypothèse de leur relation avec une épidémie de peste. Si l'on ne peut évidemment pas exclure qu'une crise de mortalité autre, non rapportée dans les sources historiques qui nous sont parvenues, puisse avoir frappé Kutná Hora durant la première moitié du XIV siècle, la Peste noire apparaît donc, en l'état des investigations, comme la cause la plus probable de ces enterrements multiples.

\section{Conclusions}

Si épidémies et famines comptent parmi les crises de mortalité qui ont laissé le plus de traces archéologiques tangibles, notamment dans le domaine funéraire, peu d'études s'étaient jusqu'alors attachées à définir les critères permettant de faire la distinction entre les sépultures relevant de l'un ou l'autre de ces contextes. L'ensemble funéraire de Sedlec, $\mathrm{du}$ fait de son attribution fiable à un horizon chronologique couvrant à la fois une famine sévère et l'épidémie de peste la plus meurtrière du Moyen Âge, offrait en ce sens une opportunité rare d'explorer cette question par le prisme de l'archéo-anthropologie.

La présente étude, en se concentrant sur l'analyse de trois tombes relevant avec certitude de la première phase d'inhumations multiples ayant été opérées sur le site, a permis de mettre en évidence certaines spécificités paléobiologiques des victimes de la crise et, sur cette base, de proposer un premier diagnostic. L'allure générale du profil de mortalité par âge avant 20 ans, la valeur de certains indices démographiques et, dans une moindre mesure, les données relatives à l'état de santé préexistant des individus, tel que documenté par certains indicateurs de stress (hypoplasies de l'émail et cribra orbitalia), font apparaître une épidémie de peste comme la cause la plus probable des décès. 
Cette hypothèse diagnostique, fondée sur une convergence d'arguments paléobiologiques, devra idéalement être confortée par une recherche d'ADN pathogène visant à identifier des vestiges moléculaires de Yersinia pestis dans les restes humains provenant des trois sépultures étudiées. Une telle identification permettrait par là même de valider la démarche méthodologique proposée pour distinguer, sur la base de l'étude anthropobiologique, un contexte de mortalité par peste d'un contexte de famine. La recherche engagée sur le site de Sedlec gagnerait par ailleurs à être poursuivie, en intégrant l'étude des autres sépultures multiples, notamment celles clairement attribuables à la seconde phase d'inhumations. Leur analyse pourrait, le cas échéant, contribuer à asseoir le premier diagnostic de peste proposé, voire fournir les uniques arguments décisifs si la recherche paléogénomique s'avérait inopérante faute d'une conservation suffisamment bonne de l'ADN.

\section{Remerciements}

L'étude des trois sépultures multiples du site de Sedlec a été réalisée dans le cadre d'un travail universitaire financé par le programme Erasmus+ de l'Union Européenne et a été soutenue par le ministère de la Culture de la République tchèque (DKRVO 2019-2023/7.II.c, 00023272). Une partie des données de comparaison ont été acquises dans le cadre d'un programme de recherche financé par l'Agence nationale de la recherche au titre du programme Investissements d'avenir (ANR-10-LABX-52). Nous remercions les chercheurs et institutions qui nous ont autorisés l'accès aux collections d'Hereford (J. Buckberry, Biological Anthropology Research Centre, Bradford), des Fédons (M. Signoli, UMR 7268 - ADES, Marseille) et de Termonde (C. Polet, Institut royal des sciences naturelles de Belgique, Bruxelles). Nous adressons enfin nos remerciements à Pascal Sellier (UMR 7206 - Eco-Anthropologie, Paris) pour les orientations bibliographiques qu'il nous a fournies concernant les travaux de démographie historique relatifs aux contextes de famine.

\section{Liens d'intérêt}

Les auteurs déclarent ne pas avoir de liens d'intérêt.

\section{Références}

Bizot B, Castex D, Reynaud P et al (2005) La saison d'une peste (avril-septembre 1590). Le cimetière des Fédons à Lambesc. CNRS Éditions, Paris, $131 \mathrm{p}$

Blanchard I (2005) Mining, Metallurgy, and Minting in the Middle Ages. Vol 2: Continuing Afro-European Supremacy, 1250-1450. Franz Steiner Verlag, Stuttgart

Bos KI, Schuenemann VJ, Golding GB et al (2011) A draft genome of Yersinia pestis from victims of the Black Death. Nature 478(7370):506-510

Bräuer G (1988) Osteometrie. In: Knussmann R (ed) Anthropologie: Handbuch der vergleichenden Biologie des Menschen. G. Fischer, Stuttgart, pp 160-232
Brázdil R, Kotyza O, Bauch M (2018) Climate and famines in the Czech lands prior to $\mathrm{AD}$ 1500: possible interconnections in a European context. In: Collet D, Schuh M (ed) Famines During the "Little Ice Age" (1300-1800). Springer, Cham, pp 91-114

Brůžek J (2002) A method for visual determination of sex, using the human hip bone. American Journal of Physical Anthropology 117:157-168

Brůžek J, Santos F, Dutailly B et al (2017) Validation and reliability of the sex estimation of the human os coxae using freely available DSP2 software for bioarchaeology and forensic anthropology. American Journal of Physical Anthropology 164: 440-449

Brzobohatá H, Frolík J, Zazvonilová E (2019) Bioarchaeology of past epidemic- and famine-related mass burials with respect to recent findings from the Czech Republic. Interdisciplinaria Archaeologica 10:79-87

Carpentier E (1962) Autour de la Peste noire : famines et épidémies dans l'histoire du XIVe siècle. Annales. Économies, sociétés, civilisations 17(6):1062-1092

Castex D (2008) Identification and interpretation of historical cemeteries linked to epidemics. In: Raoult D, Drancourt M (ed) Paleomicrobiology: Past Human Infections. Springer-Verlag, Berlin, pp 23-48

Castex D, Brůžek J, Sellier P et al (2011) Bioarchaeological study of a mortality crisis. Cemetery of St. Benedict in Prague, Czech Republic ( $17^{\text {th }}-18^{\text {th }}$ century AD): methodological approach. Anthropologie (Brno) 49:79-88

Castex D, Kacki S (2016) Demographic patterns distinctive of epidemic cemeteries in archaeological samples. In: Drancourt M, Raoult D (ed) Paleomicrobiology of Humans. Microbiology Spectrum 4:PoH-0015-2015

Castex D, Réveillas H (2007) Investigation sur une crise de mortalité à Boulogne-sur-Mer (Pas-de-Calais, XVIII ${ }^{\mathrm{e}} \mathrm{s}$.). Hypothèse d'interprétation. Bulletins et Mémoires de la Société d'Anthropologie de Paris 19:21-37

Colet A, Muntané i Santiveri JX, Ruíz Ventura J et al (2014) The Black Death and its consequences for the Jewish community in Tàrrega: Lessons from history and archeology. The Medieval Globe 1:63-96

Coqueugniot H, Weaver TD, Houët F (2010) Brief communication: A probabilistic approach to age estimation from infracranial sequences of maturation. American Journal of Physical Anthropology 142:655-664

Connell B, Redfern R, Thomas C (2012) Introduction. In: Connell B, Jones AG, Redfern R et al (ed) A bioarchaeological study of medieval burials on the site of St Mary Spital. Lavenham Press, London, pp 1-18

DeWitte SN (2006) The Paleodemography of the Black Death 1347-1351. PhD thesis, Pennsylvania State University, $253 \mathrm{p}$

DeWitte SN, Yaussy SL (2017) Femur length and famine mortality in medieval London. Bioarchaeology International 1:171-182

DeWitte SN, Yaussy SL (2020) Sex differences in adult famine mortality in medieval London. American Journal of Physical Anthropology 171:164-169

Drancourt M, Aboudharam G, Signoli M et al (1998) Detection of 400-year-old Yersinia pestis DNA in human dental pulp: an approach to the diagnosis of ancient septicemia, Proceedings of the National Academy of Sciences 95:12637-12640 
Dupâquier J (1979) L'analyse statistique des crises de mortalité. In: Charbonneau $\mathrm{H}$, Larose A (ed) Les grandes mortalités : étude méthodologique des crises démographiques du passé. UIESP, Liège, pp 83-112

Fiorato V, Boylston A, Knüsel C (2000) Blood Red Rose: The Archaeology of a Mass Grave from the Battle of Towton, AD 14610. Oxbow Books, Oxford, 277 p

Frolík J (2017) Pohřbívání ve vrcholném středověku a v novověku na Chrudimsku, Pardubicku a Kolínsku - Bestattungen im Hochmittelalter und in der Neuzeit in den Regionen Chrudim, Pardubice und Kolín. Archaeologia historica 42/1:187-205

Galanaud P, Galanaud A, Giraudoux P et al (2020) Mortality and demographic recovery in early post-black death epidemics: Role of recent emigrants in medieval Dijon. PloS one 15: e0226420

Geber J (2014) Skeletal manifestations of stress in child victims of the Great Irish Famine (1845-1852): prevalence of enamel hypoplasia, Harris lines, and growth retardation. American Journal of Physical Anthropology 155:149-161

Geber J (2015) Victims of Ireland's Great Famine. University Press of Florida, Gainesville, $285 \mathrm{p}$

Geber J (2016) Mortality among institutionalised children during the Great Famine in Ireland: bioarchaeological contextualisation of non-adult mortality rates in the Kilkenny Union Workhouse, 1846-1851. Continuity and Change 31:101-126

Geber J (2017) Interring the 'deserving' child: the archaeology of the deaths and burials of children at the Kilkenny Workhouse during the Great Famine in Ireland, 1845-52. In: Murphy E, Le Roy M (ed) Children, Death and Burial: Archaeological Discourses. Oxbow Books, Oxford, pp 249-262

Geber J, Murphy E (2012) Scurvy in the Great Irish Famine: evidence of vitamin $\mathrm{C}$ deficiency from a mid- $19^{\text {th }}$ century skeletal population. American Journal of Physical Anthropology 148: 512-524

Godde K, Pasillas V, Sanchez A (2020) Survival analysis of the Black Death: Social inequality of women and the perils of life and death in Medieval London. American Journal of Physical Anthropology 173:168-178

Goudie Falckenbach E, Ryssaert C, Brion M et al (2012) Archeologisch onderzoek in Dendermonde op de site van het voormalige Birgitinessenklooster Maria Troon. Archaeologia Mediaevalis 35:142-149

Grainger I, Hawkins D, Cowal L et al (2008) The Black Death cemetery, East Smithfield, London. MoLAS Monograph no. 43, Museum of London Archaeology Service, London, $64 \mathrm{p}$

Grumbkow PV, Zipp A, Seidenberg V et al (2011) Brief communication: Evidence of Bartonella quintana infections in skeletons of a historical mass grave in Kassel, Germany. American Journal of Physical Anthropology 146:134-137

Guinnane TW, Ó Gráda C (2002a) The workhouses and Irish famine mortality. In: Dyson T, Ó Gráda C (ed) Famine Demography: Perspectives from the Past and Present. Oxford University Press, Oxford, pp 44-64

Guinnane TW, Ó Gráda C (2002b) Mortality in the North Dublin Union during the Great Famine. The Economic History Review 55:487-506

Haensch S, Bianucci R, Signoli M et al (2010) Distinct clones of Yersinia pestis caused the Black Death. PLoS Pathogens 6: e1001134
Henry L (1980) Techniques d'analyse en démographie historique. Ined, Paris, $180 \mathrm{p}$

Hollingsworth M, Hollingsworth T (1971) Plague mortality rates by age and sex in the parish of St. Botolph's without Bishopsgate, London, 1603. Population Studies 25:131-146

Jones AG, Walker D (2012) Demography. In: Connell B, Jones AG, Redfern R, et al (ed) A bioarchaeological study of medieval burials on the site of St Mary Spital. Lavenham Press, London, pp 25-35

Kacki S (2016) Influence de l'état sanitaire des populations anciennes sur la mortalité en temps de peste : contribution à la paléoépidémiologie. Thèse de doctorat, Université de Bordeaux, $749 \mathrm{p}$

Kacki S (2017) Influence de l'état sanitaire des populations du passé sur la mortalité en temps de peste : contribution à la paléoépidémiologie. Bulletins et Mémoires de la Société d'Anthropologie de Paris 29:202-212

Kacki S (2020) Digging up the victims of the Black Death: a bioarchaeological perspective on the second plague pandemic. In: Gerrard C, Forlin P, Brown P (ed) Waiting for the End of the World? New Perspectives on Natural Disasters in Medieval Europe. Society for Medieval Archaeology Monographs no. 43, Routledge, London, pp 259-279

Kozlov I, Samsonova A (2005) The impact of the siege on the physical development of children. In: Barber J (ed) Life and Death in Leningrad, 1914-44. Palgrave Macmillan publishers, London, pp 174-196

Ledermann S (1969) Nouvelles tables-types de mortalité. Ined, Travaux et documents 53, Presses universitaires de France, Paris, $260 \mathrm{p}$

Loe L, Barker C, Wright R (2013) An osteological profile of trench warfare. Peri-mortem trauma sustained by soldiers who fought and died in the battle of Fromelles, 1916. In: Knüsel C, Smith M (dir) The Routledge Handbook of the Bioarchaeology of Human Conflict. Routledge, London, pp 575-601

Lucas HS (1930) The Great European Famine of 1315, 1316, and 1317. Speculum 5:343-377

Lütgert SA (2000) Victims of the Great famine and the Black Death? The archaeology of the mass graves found in the former graveyard of Holy Ghost Hospital, Lubeck (N. Germany), in the European Context. Hikuin 27:255-264

MacIntyre K (2002) Famine and the female mortality advantage. In: Dyson T, Ó Gráda C (ed) Famine Demography: Perspectives from the Past and Present. Oxford University Press, Oxford, pp 240-259

Mallet E (1835) Notice sur les anciennes pestes de Genève. Annales d'hygiène publique et de médecine légale 14:206-221

Mengel DC (2011) A plague on Bohemia? Mapping the Black Death. Past \& Present 211:3-34

Mensforth RP, Lovejoy CO, Lallo JW et al (1978) The role of constitutional factors, diet, and infectious disease in the etiology of porotic hyperostosis and periosteal reactions in prehistoric infants and children. Medical Anthropology 2:1-59

Moorrees CFA, Fanning EA, Hunt EE (1963) Age variation of formation stages for ten permanent teeth. Dental Research 6: $1490-1502$ 
Ó Gráda C. 2009. Famine: A short history. Princeton University Press, Princeton, $327 \mathrm{p}$

Owings Webb PA, Suchey JM (1985) Epiphyseal union of the anterior iliac crest and medial clavicle in a modern multiracial sample of American males and females. American Journal of Physical Anthropology 68:457-466

Pany D (2003) An analysis of occupationally-induced stress markers on the skeletal remains from the ancient Hallstatt cemetery. $\mathrm{PhD}$ thesis, University of Vienna, $153 \mathrm{p}$

Pitkänen KJ (2002) Famine mortality in nineteenth-century Finland: is there a sex bias? In: Dyson T, Ó Gráda C (ed) Famine Demography: Perspectives from the Past and Present. Oxford University Press, Oxford, pp 65-92

Sellier P (1995) Paléodémographie et archéologie funéraire : les cimetières de Mehrgarh, Pakistan. Paléorient 21:123-143

Sellier P (1996) La mise en évidence d'anomalies démographiques et leur interprétation : population, recrutement et pratiques funéraires du tumulus de Courtesoult. In: Piningre J-F (ed) Nécropoles et sociétés au premier âge du Fer : le tumulus de Courtesoult (Haute-Saône). Documents d'Archéologie Française 54, Éditions de la Maison des Sciences de l'Homme, Paris, pp 188-202

Séguy I, Buchet L (2011) Manuel de paléodémographie. Ined, Paris, $288 \mathrm{p}$

Séguy I, Pennec S, Tzortzis S et al (2006) Modélisation de l'impact de la peste à travers l'exemple de Martigues (Bouches-duRhône). In: Buchet L, Dauphin C, Séguy I (dir) La paléodémographie. Mémoire d'os, mémoire d'hommes. Actes des 8 journées anthropologiques de Valbonne. Antibes, Éditions APDCA, Antibes, pp 323-331

Signoli M, Séguy I, Biraben J-N et al (2002) Paleodemography and historical demography in the context of an epidemic: plague in Provence in the eighteenth century. Population 57:829-854

Signoli M, Tzortzis S, Bizot B et al (2007) Découverte d'un cimetière de pestiférés du XVII ${ }^{\mathrm{e}}$ siècle (Puy-Saint-Pierre, Hautes-
Alpes, France). In: Signoli M, Chevé D, Adalian P et al (ed) Peste : entre épidémies et sociétés. Firenze University Press, Florence, pp 131-136

Silventoinen K (2003) Determinants of variation in adult body height. Journal of Biosocial Science 35:263-285

Spyrou MA, Keller M, Tukhbatova R et al (2019). Phylogeography of the second plague pandemic revealed through analysis of historical Yersinia pestis genomes. Nature communications 10: 4470

Steckel RH (1995) Stature and the standard of living. Journal of Economic Literature 33:1903-1940

Stuart-Macadam P (1985) Porotic hyperostosis: representative of a childhood condition. American Journal of Physical Anthropology 66:391-398

Stloukal M, Hanáková H (1978) Die lange der Langsknochen altslawischer Bovolkerungen Unter besonder Berucksichistigung von Wachstumsfragen. Homo 29:53-69

Turpeinen O (1997) Mortality and causes of death in Helsinki in 1750-1865 with a comparison with Tallin. Finnish Yearbook of Population Research 34:47-70

Tzortzis S (2009) Archives biologiques et archives historiques, une approche anthropologique de l'épidémie de peste de 1720-1721 à Martigues (Bouches-du-Rhône, France). Thèse de doctorat, Aix-Marseille Université, $501 \mathrm{p}$

Wescott DJ, Baker LE, Wernecke DC et al (2012) A mass grave of Mexican soldiers from the Resaca de la Palma battlefield (41CF3): Demography and battle-related injuries. Bulletin of Texas Archaeology 83:1-21

Yaussy SL, DeWitte SN (2018) Patterns of frailty in non-adults from medieval London. International Journal of Paleopathology 22:1-7

Yaussy SL, DeWitte SN, Redfern RC (2016) Frailty and famine: patterns of mortality and physiological stress among victims of famine in medieval London. American Journal of Physical Anthropology 160:272-283 


\section{Annexes}

\section{Annexe 1. Informations additionnelles sur les données de comparaison}

Les quotients de mortalités utilisés dans l'analyse comparative ont été calculés par les auteurs sur la base des effectifs de décès par classes d'âge rapportés pour différents sites archéologiques et dans différents travaux de démographie historiques (cf. tableau 2 du corps de l'article). Les prévalences des indicateurs de stress prises en compte dans les confrontations sont directement celles rapportées dans les publications consultées.

Concernant les contextes de peste, quatre sites funéraires ont été intégrés à l'analyse. L'attribution des sépultures à une épidémie de peste est démontrée par les résultats d'analyses paléomicrobiologiques pour les sites d'East Smithfield (Bos et al., 2011), d'Hereford Cathedral (Haensch et al., 2010) et des Fédons (Drancourt et al., 1998). La relation entre le cimetière de Maria Troon (Termonde) et une épidémie de peste n'est à ce jour attestée que par des sources textuelles
(Goudie Falckenbach et al., 2012), les premières analyses paléogénomiques menées sur les vestiges squelettiques n'ayant pas permis de détecter des séquences d'ADN spécifiques à l'agent pathogène Yersinia pestis. Les données biologiques individuelles se rapportant à ces quatre sites sont disponibles dans deux thèses de doctorat (DeWitte, 2006 ; Kacki, 2016) consultables en ligne (série d'East Smithfiled; https://etda.libraries.psu.edu/files/final_submissions/5714; séries d'Hereford Cathedral, des Fédons et de Maria Troon : https://tel.archives-ouvertes.fr/tel-01370446/document). Les données de démographie historique concernant les épidémies de peste de Londres et de Genève sont, pour leur part, issues de publications livrant les effectifs de décès par classe d'âge durant ces crises (Mallet, 1835 ; Hollingsworth et Hollingsworth, 1971). Les effectifs de décès et quotients de mortalité pour chaque site ou localité sont rapportés dans les tableaux S1 et S2.

Concernant les contextes de famines, deux sites archéologiques ont pu être intégrés à l'étude. Pour le premier d'entre eux (St. Mary Graces, Londres), ont été pris en compte dans l'analyse les squelettes issus des sépultures multiples de

\begin{tabular}{|l|c|c|}
\hline \multirow{2}{*}{$\begin{array}{c}\text { Classe } \\
\text { d'âge }\end{array}$} & \multicolumn{2}{|c|}{ East Smithfield } \\
\cline { 2 - 3 } & $\mathrm{N}$ & ${ }_{\mathrm{a}} \mathrm{q}_{\mathrm{x}}(\% \mathrm{o})$ \\
\hline$[0]$ & 6 & 12,4 \\
\hline$[1-4]$ & 31 & 64,7 \\
\hline$[5-9]$ & 45 & 100,4 \\
\hline$[10-14]$ & 66 & 163,8 \\
\hline$[15-19]$ & 86 & 255,2 \\
\hline$[20+]$ & 251 & 1000,0 \\
\hline Total & $\mathbf{4 8 5}$ & - \\
\hline
\end{tabular}

\begin{tabular}{|c|c|}
\hline \multicolumn{2}{|c|}{ Hereford Cathedral } \\
\hline $\mathrm{N}$ & ${ }_{\mathrm{a}} \mathrm{q}_{\mathrm{x}}(\% \mathrm{o})$ \\
\hline 5 & 27,0 \\
\hline 23 & 127,8 \\
\hline 28 & 178,3 \\
\hline 14 & 108,5 \\
\hline 9 & 78,3 \\
\hline 106 & 1000,0 \\
\hline $\mathbf{1 8 5}$ & - \\
\hline
\end{tabular}

\begin{tabular}{|c|c|}
\hline \multicolumn{2}{|c|}{ Les Fédons } \\
\hline $\mathrm{N}$ & ${ }_{\mathrm{a}} \mathrm{q}_{\mathrm{x}}(\% \mathrm{o})$ \\
\hline 4 & 30,1 \\
\hline 19 & 147,3 \\
\hline 24 & 218,2 \\
\hline 19 & 220,9 \\
\hline 7 & 104,5 \\
\hline 60 & 1000,0 \\
\hline $\mathbf{1 3 3}$ & - \\
\hline
\end{tabular}

\begin{tabular}{|c|c|}
\hline \multicolumn{2}{|c|}{ Maria Troon } \\
\hline $\mathrm{N}$ & ${ }_{\mathrm{a}} \mathrm{q}_{\mathrm{x}}(\%)$ \\
\hline 0 & 0,0 \\
\hline 5 & 50,5 \\
\hline 22 & 234,0 \\
\hline 17 & 236,1 \\
\hline 11 & 200,0 \\
\hline 44 & 1000,0 \\
\hline $\mathbf{9 9}$ & - \\
\hline
\end{tabular}

Tableau S1. Effectifs des décès $(\mathrm{N})$ et quotients de mortalité par classes d'âge $\left({ }_{\mathrm{a}} \mathrm{q}_{\mathrm{x}}\right)$ pour les sites d'inhumations de pestiférés intégrés au corpus de comparaison (d'après les données de DeWitte, 2006 ; Kacki, 2016) / Number of deaths (N) and mortality quotients $\left.{ }_{(a} q_{x}\right)$ by age group in the plague burial sites from the comparison dataset (based on data from DeWitte, 2006; Kacki, 2016)

\begin{tabular}{|l|c|c|}
\hline \multirow{2}{*}{$\begin{array}{c}\text { Classe } \\
\text { d'âge }\end{array}$} & \multicolumn{2}{|c|}{ St. Botolph, Londres } \\
\cline { 2 - 3 } & $\mathrm{N}$ & ${ }_{\mathrm{a}} \mathrm{q}_{\mathrm{x}}(\% \mathrm{o})$ \\
\hline$[0]$ & 127 & 89,3 \\
\hline$[1-4]$ & 224 & 173,0 \\
\hline$[5-9]$ & 242 & 226,0 \\
\hline$[10-14]$ & 137 & 165,3 \\
\hline$[15-19]$ & 148 & 213,9 \\
\hline$[20+]$ & 544 & 1000,0 \\
\hline Total & $\mathbf{1 4 2 2}$ & - \\
\hline
\end{tabular}

\begin{tabular}{|c|c|}
\hline \multicolumn{2}{|c|}{ Genève } \\
\hline $\mathrm{N}$ & ${ }_{\mathrm{a}} \mathrm{q}_{\mathrm{x}}(\%)$ \\
\hline 48 & 27,9 \\
\hline 199 & 119,2 \\
\hline 210 & 142,8 \\
\hline 179 & 142,0 \\
\hline 199 & 183,9 \\
\hline 883 & 1000,0 \\
\hline $\mathbf{1 7 1 8}$ & - \\
\hline
\end{tabular}

Tableau S2. Effectifs des décès $(\mathrm{N})$ et quotients de mortalité par classes d’âge $\left({ }_{\mathrm{a}} \mathrm{q}_{\mathrm{x}}\right)$ enregistrés en contexte d'épidémies de peste pour la paroisse londonienne de St. Botolph (1603) et la ville de Genève (XVII e siècle) (d'après les données de Hollingsworth et Hollingsworth, 1971 et Mallet, 1835) / Number of deaths ( $N)$ and mortality quotients ( $\left.{ }_{a} q_{x}\right)$ by age group recorded during plague epidemics in the parish of St. Botolph, London (1603) and the city of Geneva (17th century) (based on data from Hollingsworth and Hollingsworth, 1971 and Mallet, 1835) 
Type D (selon la classification adoptée par les archéologues en charge de la fouille) et se rattachant aux périodes 14 (1120-1200), 15 (1200-1250) et 17 (1400-1539) de l'occupation du site (Connell et al., 2012). Le lien de ces tombes avec des épisodes de famine est suspecté sur la base de données historiques relatant l'occurrence de crises frumentaires à ces périodes (Connell et al., 2012). Pour ce site, seules ont pu être exploitées les données concernant la fréquence des indicateurs de stress. Le second site funéraire, situé dans l'enceinte de l'ancienne maison de travail de Kilkenny, a livré des sépultures multiples dont le lien avec la Grande Famine Irlandaise (1845-1852) est attesté par les documents d'archives de l'établissement (Geber, 2016 ; 2017). Les données concernant la mortalité par classes d'âge et la fréquence des indicateurs de stress sont issues de l'étude monographique du site (Geber, 2015). Dans cette dernière, les grands adolescents de plus de 18 ans ont été classés comme jeunes adultes (35 individus décédés entre 18 et 25 ans). Afin de palier l'impact de cette différence de mode de classement sur les résultats de la confrontation avec les données du site de Sedlec, ces sujets ont été ventilés entre la classe d'âge [15-19] $(\mathrm{n}=10)$ et le groupe des plus de 20 ans $(n=25)$, en postulant une distribution homogène des décès à chaque âge couvert par l'intervalle d'origine et au prorata du nombre d'années se rapportant aux groupes cibles. Les effectifs de décès par classe d'âge tenant compte de cette opération et les quotients de mortalité correspondants sont présentés dans le tableau S3.

Ces données archéologiques ont été complétées par des informations issues de travaux de démographie historique concernant, d'une part, la Grande famine irlandaise et, d'autre part, deux épisodes de famine survenus au XIX ${ }^{\mathrm{e}}$ siècle en Finlande. Les données acquises pour la première de ces crises, reproduites dans le tableau S4, correspondent aux décès enregistrés tant pour les maisons de travail qu'en dehors de celles-ci dans le recensement de 1851, lequel couvre une période d'une décennie (Guinnane et Ó Gráda, 2002a). Il est à noter que si la majeure partie des décès enregistrés pour les maisons de travail sont attribuables à la

\begin{tabular}{|l|c|c|}
\hline \multirow{2}{*}{$\begin{array}{c}\text { Classe } \\
\text { d'âge }\end{array}$} & \multicolumn{2}{|c|}{ Kilkenny Union House } \\
\cline { 2 - 3 } & $\mathrm{N}$ & ${ }_{\mathrm{a}} \mathrm{q}_{\mathrm{x}}(\% \mathbf{0})$ \\
\hline$[0]$ & 95 & 97,9 \\
\hline$[1-4]$ & 208 & 237,7 \\
\hline$[5-9]$ & 151 & 226,4 \\
\hline$[10-14]$ & 68 & 131,8 \\
\hline$[15-19]$ & 33 & 73,7 \\
\hline$[20+]$ & 415 & 1000,0 \\
\hline Total & $\mathbf{9 7 0}$ & - \\
\hline
\end{tabular}

Tableau S3. Effectifs des décès $(\mathrm{N})$ et quotients de mortalité par classes d'âge $\left({ }_{a} q_{x}\right)$ pour le site de Kilkenny Union Workhouse (d'après les données de Geber, 2015) / Number of deaths (N) and mortality quotients $\left({ }_{a} q_{x}\right)$ by age group in the Kilkenny Union Workhouse burial site (based on data from Geber, 2015)

famine, car postérieurs à 1846, une part plus importante des décès enregistrés hors de ces établissements est intervenue avant cette date, de sorte que les effectifs et quotients de mortalité ne rendent qu'imparfaitement compte de la mortalité par famine. Les données relatives aux crises frumentaires qui ont frappé la Finlande durant les années 1832-1833 et 1857-1858 sont, elles, issues d'un travail de démographie historique rapportant le taux de mortalité par classes d'âge durant ces épisodes et ceux relevées hors années de famine durant la même décennie (Pitkänen, 2002). Les effectifs de décès n'étant pas fournis dans cette étude, les données autorisent uniquement le calcul de l'accroissement du taux de mortalité spécifique à chaque classe d'âge dans ces contextes. Afin d'en inférer des quotients de mortalité, un second jeu de données a été utilisé pour définir la distribution par âge des décès théorique hors temps de crise. Ce référentiel a été constitué en sommant les décès enregistrés sur les décennies correspondantes (hors années de famine) pour la ville d'Helsinki (Turpeinen, 1997). Les effectifs de décès hors temps de crise frumentaire, le taux d'accroissement lors des deux épisodes de famines et les quotients de mortalité calculés sur cette base sont fournis dans les tableaux S5- S6.

\begin{tabular}{|l|c|c|}
\hline \multirow{2}{*}{\begin{tabular}{c}
\multirow{2}{*}{$\begin{array}{c}\text { Classe } \\
\text { d'âge }\end{array}$} \\
\cline { 2 - 3 }
\end{tabular}} & \multicolumn{2}{|c|}{$\begin{array}{c}\text { Décès } \\
\text { workhouses }\end{array}$} \\
\cline { 2 - 3 }$[0]$ & $\mathrm{N}$ & ${ }_{\mathrm{a}} \mathrm{q}_{\mathrm{x}}(\% \mathrm{o})$ \\
\hline$[1-4]$ & 46964 & 77,1 \\
\hline$[5-9]$ & 41356 & 171,0 \\
\hline$[10-14]$ & 29760 & 152,1 \\
\hline$[15-19]$ & 13793 & 109,5 \\
\hline$[20+]$ & 119515 & 50,7 \\
\hline Total & 271884 & 1000,0 \\
\hline
\end{tabular}

\begin{tabular}{|c|c|}
\hline \multicolumn{2}{|c|}{$\begin{array}{c}\text { Décès } \\
\text { hors workhouses }\end{array}$} \\
\hline $\mathrm{N}$ & ${ }_{\mathrm{a}} \mathrm{q}_{\mathrm{x}}(\% \mathrm{o})$ \\
\hline 121839 & 117,1 \\
\hline 189584 & 182,2 \\
\hline 85249 & 81,9 \\
\hline 48156 & 46,3 \\
\hline 52715 & 50,7 \\
\hline 542784 & 1000,0 \\
\hline 1040327 & - \\
\hline
\end{tabular}

\begin{tabular}{|c|c|}
\hline \multicolumn{2}{|c|}{$\begin{array}{c}\text { Décès } \\
\text { totaux }\end{array}$} \\
\hline $\mathrm{N}$ & ${ }_{\mathrm{a}} \mathrm{q}_{\mathrm{x}}(\%)$ \\
\hline 142803 & 108,8 \\
\hline 236080 & 179,9 \\
\hline 126605 & 96,5 \\
\hline 77916 & 59,4 \\
\hline 66508 & 50,7 \\
\hline 662299 & 1000,0 \\
\hline 1312211 & - \\
\hline
\end{tabular}

Tableau S4. Effectifs des décès $(\mathrm{N})$ et quotients de mortalité par classes d'âge $\left({ }_{\mathrm{a}} \mathrm{q}_{\mathrm{x}}\right)$ enregistrés en Irlande sur la période 18411851, dans les maisons de travail (workhouses) et hors de celles-ci (d'après les données de Guinnane et Ó Gráda, 2002a) / Number of deaths (N) and mortality quotients $\left({ }_{a} q_{x}\right)$ by age group recorded in Ireland over the period 1841-1851, in and out of workhouses (based on data from Guinnane and Ó Gráda, 2002a) 


\begin{tabular}{|c|c|c|c|c|c|c|}
\hline \multirow{2}{*}{ Classe d'âge } & \multicolumn{3}{|c|}{ Mortalité naturelle Helsinki } & \multicolumn{3}{|c|}{ Mortalité famine 1832-1833 } \\
\hline & $\mathrm{N}$ & $\mathrm{D}_{\mathrm{x}}$ & ${ }_{\mathrm{a}} \mathrm{q}_{\mathrm{x}}(\% \mathrm{o})$ & $\Delta$ & $\mathrm{D}_{\mathrm{x}}$ & ${ }_{a} q_{x}(\% 0)$ \\
\hline [0] & 1010 & 372,7 & 229,2 & 1,40 & 138,0 & 138,0 \\
\hline$[1-4]$ & 686 & 253,1 & 202,0 & 2,88 & 192,9 & 223,8 \\
\hline [5-9] & 162 & 59,8 & 59,8 & 4,62 & 73,1 & 109,3 \\
\hline$[10-14]$ & 85 & 31,4 & 33,4 & 4,21 & 35,0 & 58,8 \\
\hline [15-19] & 91 & 33,6 & 36,9 & 3,02 & 26,8 & 47,9 \\
\hline [20-24] & 317 & 117,0 & 133,6 & 2,48 & 76,7 & 143,6 \\
\hline [25-29] & 374 & 138,0 & 182,0 & 2,27 & 82,9 & 181,3 \\
\hline [30-34] & 424 & 156,5 & 252,2 & 2,45 & 101,6 & 271,2 \\
\hline [35-39] & 339 & 125,1 & 269,7 & 2,42 & 80,1 & 293,3 \\
\hline [40-44] & 226 & 83,4 & 246,2 & 2,27 & 50,2 & 260,1 \\
\hline [45-49] & 157 & 57,9 & 226,9 & 2,61 & 40,0 & 280,2 \\
\hline [50-54] & 127 & 46,9 & 237,4 & 2,38 & 29,6 & 287,7 \\
\hline [55-59] & 102 & 37,6 & 250,0 & 2,13 & 21,2 & 290,2 \\
\hline [60-64] & 89 & 32,8 & 290,8 & 2,00 & 17,4 & 334,7 \\
\hline [65-69] & 79 & 29,2 & 364,1 & 1,79 & 13,9 & 401,3 \\
\hline [70-74] & 66 & 24,4 & 478,3 & 1,68 & 10,8 & 524,5 \\
\hline$[75+]$ & 72 & 26,6 & 1000,0 & 1,40 & 9,8 & 1000,0 \\
\hline Total & 2710 & 1000,0 & - & - & 1000,0 & - \\
\hline
\end{tabular}

Tableau S5. Effectifs des décès par classe d'âge - absolus $(\mathrm{N})$ et pour 1000 décès $\left(\mathrm{D}_{\mathrm{x}}\right)$ - à Helsinki durant les années 1830 (hors années de famine), taux d'accroissement $(\Delta)$ de la mortalité par classe d'âge lors de la famine de 1832-1833 et quotients de mortalité par classes d'âge $\left({ }_{a} q_{\mathrm{x}}\right)$ durant cette crise (d'après les données de Turpeinen, 1997 et Pitkänen, 2002) / Number of deaths - absolute (N) and for 1,000 deaths - in Helsinki during the 1830s (excluding famine years), rate of increase ( $\Delta$ ) in age-specific mortality rate during the famine of 1832-1833, and mortality quotients by age group $\left({ }_{a} q_{x}\right)$ during this crisis (based on data from Turpeinen, 1997 and Pitkänen, 2002)

\begin{tabular}{|l|c|c|c|}
\hline \multirow{2}{*}{ Classe d'âge } & \multicolumn{3}{|c|}{ Mortalité naturelle Helsinki } \\
\cline { 2 - 4 } & $\mathrm{N}$ & $\mathrm{D}_{\mathrm{x}}$ & ${ }_{\mathrm{a}} \mathrm{q}_{\mathrm{x}}(\%)$ \\
\hline$[0]$ & 1665 & 328,3 & 203,5 \\
\hline$[1-4]$ & 1444 & 284,7 & 221,6 \\
\hline$[5-9]$ & 323 & 63,7 & 63,7 \\
\hline$[10-14]$ & 172 & 33,9 & 36,2 \\
\hline$[15-19]$ & 227 & 44,8 & 49,6 \\
\hline$[20-24]$ & 475 & 93,7 & 109,2 \\
\hline$[25-29]$ & 642 & 126,6 & 165,7 \\
\hline$[30-34]$ & 621 & 122,4 & 192,1 \\
\hline$[35-39]$ & 556 & 109,6 & 212,9 \\
\hline$[40-44]$ & 454 & 89,5 & 220,8 \\
\hline$[45-49]$ & 373 & 73,5 & 232,8 \\
\hline$[50-54]$ & 305 & 60,1 & 248,2 \\
\hline$[55-59]$ & 249 & 49,1 & 269,5 \\
\hline$[60-64]$ & 253 & 49,9 & 374,8 \\
\hline$[65-69]$ & 155 & 30,6 & 367,3 \\
\hline$[70-74]$ & 122 & 24,1 & 456,9 \\
\hline$[75+]$ & 145 & 28,6 & 1000,0 \\
\hline Total & $\mathbf{5 0 7 2}$ & $\mathbf{1 0 0 0 , 0}$ & - \\
\hline
\end{tabular}

\begin{tabular}{|c|c|c|}
\hline \multicolumn{3}{|c|}{ Mortalité famine 1857-58 } \\
\hline$\Delta$ & $\mathrm{D}_{\mathrm{x}}$ & ${ }_{\mathrm{a}} \mathrm{q}_{\mathrm{x}}(\% \mathbf{})$ \\
\hline 1,15 & 158,9 & 158,9 \\
\hline 1,73 & 207,4 & 246,6 \\
\hline 2,10 & 56,5 & 89,1 \\
\hline 1,92 & 27,5 & 47,7 \\
\hline 1,67 & 31,6 & 57,5 \\
\hline 1,65 & 65,4 & 126,2 \\
\hline 1,42 & 76,0 & 167,8 \\
\hline 1,41 & 73,0 & 193,8 \\
\hline 1,41 & 65,3 & 214,9 \\
\hline 1,64 & 62,0 & 259,9 \\
\hline 1,56 & 48,4 & 274,5 \\
\hline 1,36 & 34,6 & 270,4 \\
\hline 1,25 & 25,9 & 277,5 \\
\hline 1,25 & 26,3 & 390,2 \\
\hline 1,16 & 14,9 & 362,8 \\
\hline 1,21 & 12,3 & 470,1 \\
\hline 1,15 & 13,9 & 1000,0 \\
\hline- & 1000,0 & - \\
\hline
\end{tabular}

Tableau S6. Effectifs des décès par classe d'âge - absolus $(N)$ et pour 1000 décès $\left(D_{x}\right)$ - à Helsinki durant les années $1850($ hors années de famine), taux d'accroissement $(\Delta)$ de la mortalité par classe d'âge lors de la famine de 1857-1858 et quotients de mortalité par classes d'âge $\left({ }_{\mathrm{a}} \mathrm{q}_{\mathrm{x}}\right.$ ) durant cette crise (d'après les données de Turpeinen, 1997 et Pitkänen, 2002) / Number of deaths - absolute (N) and for 1,000 deaths - in Helsinki during the 1830s (excluding famine years), rate of increase ( $\Delta$ ) in age-specific mortality rate during the famine of 1832-1833, and mortality quotients by age group (a $q_{x}$ ) during this crisis (based on data from Turpeinen, 1997 and Pitkänen, 2002) 
Annexe 2A. Données paléobiologiques individuelles pour l'échantillon de Sedlec (téléchargeable sur https://journals.openedition.org/bmsap/7739?file=1)

\begin{tabular}{|c|c|c|c|c|c|c|c|c|c|c|c|c|c|c|c|c|c|c|c|c|c|c|c|c|c|c|c|c|c|c|c|c|c|c|c|c|c|c|c|}
\hline 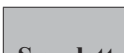 & & Âge & & & & & popla & isie $\mathrm{m}$ & naxill & aire & droi & & & & ypop & blasie & ma & xillai & ire ga & auche & & & Нуро & oplas & ie ma & tandit & ibule & droi & & & & popl & lasie & & ndibu & ule ga & auche & & plasie \\
\hline squelette & Categorie & Estimation & Classe & & M3 & M2 & M1 & P2 & P1 & $\mathrm{C}$ & 12 & I & & & 12 & C & P1 & P2 & M1 & M2 & M3 & M3 & M2 & M1 & P2 & P1 & C & 12 & \begin{tabular}{l|l}
2 & I \\
\end{tabular} & 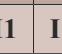 & 11 I 11 & 12 & C & P1 & P2 & M1 & M2 & M3 & individu \\
\hline 585 & $\mathrm{AD}$ & $>15$ ans & {$[20+]$} & I & & & A & A & A & A & & $\mathrm{B}$ & & & & A & A & A & & A & A & & & A & & $\mathrm{A}$ & $\mathrm{A}$ & & & & & & A & & & $\mathrm{NO}$ & A & A & B \\
\hline 586 & $x_{-1}$ & $13-17$ ans & {$[10-14]$} & 1 & A & A & $\mathrm{A}$ & & $\mathrm{A}$ & & A & $\mathrm{B}$ & 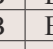 & B & A & B & B & A & A & $\mathrm{A}$ & $\mathrm{A}$ & A & $\mathrm{A}$ & A & A & $\mathrm{A}$ & $\mathrm{A}$ & B & & -1 & B & & A & A & A & A & $\mathrm{A}$ & $\mathrm{A}$ & B \\
\hline 608 & DD & $20-39$ ans & {$[20+]$} & & & & & & - & & & & & & & & & 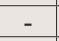 & & & & & & & & & & & & & & & & & & & & & \\
\hline 60 & D & $8,33-10$ ans & {$[5-9]$} & I & - & A & A & A & A & A & A & A & 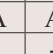 & A & A & A & A & A & A & A & - & - & A & A & - & A & A & A & $\mathrm{A} A$ & $A F$ & A & A & A & A & - & A & -1 & $x^{-}$ & A \\
\hline$\frac{610}{625}$ & $\frac{A D}{A D}$ & $\begin{array}{l}20-25 \text { ans } \\
>15 \text { ans }\end{array}$ & {$\left[\begin{array}{l}{[20-29]} \\
{[20+1}\end{array}\right.$} & $\frac{1}{1}$ & $\bar{A}$ & $\overline{\mathrm{A}}$ & $\dot{\mathrm{A}}$ & À & A & $\overline{\mathrm{A}}$ & $\bar{A}$ & $\bar{A}$ &. & - & $\overline{\mathrm{A}}$ & $\overline{\mathrm{B}}$ & $\overline{\mathrm{A}}$ & $\bar{A}$ & - & - & NO & - & - & - & - & - & - & - & $-1-$ & 5 & - & -1 & - & - & - & - & - & - & B \\
\hline & IM & $15-18$ ans & {$[15-19]$} & I & - & - & - & - & - & - & & & & - & - & & - & - & & 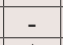 & - & - & - & - & - & - & - & & & & & & & - & - & - & -5 & - & B \\
\hline 627 & $\mathrm{AD}$ & $>20$ ans & {$[20+]$} & 1 & - & NO & NO & A & A & $\mathrm{B}$ & $\mathrm{NC}$ & B & & B & $\frac{\pi}{4}$ & $\mathrm{~B}$ & A & $\mathrm{A}$ & $\mathrm{A}$ & $\mathrm{A}$ & - & A & NO & & A & $\mathrm{B}$ & NO & $\mathrm{NC}$ & & & & & $\mathrm{SO}$ & C & A & - & NO & A & 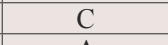 \\
\hline$\frac{643}{644}$ & $\frac{\mathrm{IM}}{\mathrm{AD}}$ & $\frac{10-12 \text { ans }}{21-27 \text { ans }}$ & {$[10-14]$} & $\frac{\mathrm{I}}{\mathrm{M}}$ & - & A & A & & A & A & & & & & & & & & - & - & - & - & & & & & & & & & & & & & & & & $-\sigma_{2}$ & A \\
\hline & IM & $5-14$ ans & {$[5-9]$} & & & - & - & - & - & - & & - & & & & & & - & - & - & $\begin{array}{lll}- \\
-\end{array}$ & - & - & - & & & - & - & & & & & & & - & - & - & & \\
\hline $758 \mathrm{~b}$ & $\mathrm{AD}$ & $>15$ ans & {$[20+]$} & I & - & - & - & - & - & - & - & - & & - & - & - & - & - & - & - & - & - & - & $1-$ & $1-2$ & - & - & - & - &.- & - & - & - & - & - & - & - & - & - \\
\hline $\begin{array}{ll}760 \\
777\end{array}$ & $\frac{\mathrm{AD}}{\mathrm{AD}}$ & $20-49$ ans & {$[30+]$} & $\frac{\mathrm{M}}{\mathrm{M}}$ & - & - & - & - & - & - & - & - & - & - & - & - & - & - & - & - & - & i & - & - & i & $\therefore$ & 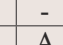 & & & $\therefore$ & $a_{2}$ & & - & - & - & - & - & - & 1 \\
\hline 778 & $\frac{\mathrm{AD}}{\mathrm{AD}}$ & $\begin{array}{l}20-39 \text { ans } \\
>40 \text { ans }\end{array}$ & & $\mathrm{M}$ & & $-\dot{s}, \quad$ & & -5 & - & -5 & & - & & - & - & & $\therefore$ & - & - & -5 & - & A & & & & A & A & & & & $A$ & & & & A & A & A & & \\
\hline 799 & IM & $\begin{array}{l}14-17 \text { ans } \\
\text { ans }\end{array}$ & {$[15-19]$} & $\frac{1}{I}$ & & - & & & 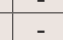 & & & & & & & & & & - & & & & & & & & & & & & & & & & & & & & \\
\hline 800 & $\mathrm{AD}$ & 1,1, a & {$[20+]$} & +2 & A & - & & $\mathrm{A}$ & $\mathrm{A}$ & NO & A & A & & No & $\mathrm{A}_{\mathrm{A}}$ & No & $\mathrm{A}$ & - & - & - & A & NO & $\mathrm{A}$ & NO & $\mathrm{A}$ & B & B & A & $\mathrm{NS}$ & IO N & No & $\bar{A}$ & No & B & A & A & NO & NO & B \\
\hline 801 & $\mathrm{IM}$ & $13-17$ ans & {$[10-14]$} & I & 2 & - & - & - & - & - & & - & & - & & 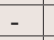 & - & -1 & - & - & - & - & - & - & - & & & & -1 & $-T$ & - & & - & & - & - & -1 & & \\
\hline 809 & $\mathrm{AD}$ & $20-22$ ans & [20-29] & M & & & & & & & & & & & & & & & & & - & & & & & & & & & & & & & & & 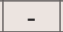 & & & \\
\hline 810 & $\mathrm{AD}$ & $20-49$ ans & {$[20+]$} & I & A & A & A & A & A & A & & A & & A & A & A & A & A & A & A & $\begin{array}{ll}- \\
-\end{array}$ & A & A & A & A & A & A & A & A & $t^{\prime}$ & A & A & A & A & A & A & A & A & $A$ \\
\hline 810 & $\frac{\mathrm{IM}}{\mathrm{IM}}$ & \begin{tabular}{|l|}
$, 5-1,25$ ans \\
$, 5-5,75$ ans
\end{tabular} & {$\left[\begin{array}{ll}{[0]} \\
{[1-4]}\end{array}\right.$} & $\frac{1}{I}$ & - & $\because$ & - & - & - & - & - & - & 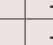 & - & - & $\overline{-}$ & - & - & - & - & - & - & $\mathrm{A}$ & $\mathrm{A}$ & A & $\mathrm{A}$ & $\overline{\mathrm{A}}$ & - & $\bar{B}$ & 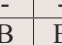 & $\overline{\mathrm{B}}$ & - & $\begin{array}{c}- \\
-\end{array}$ & - & $\bar{A}$ & A & A & - & B \\
\hline 820 & $\mathrm{AD}$ & $>20$ ans & {$[20+]$} & $\mathrm{I}$ & - & NO & & A & - & NO & A & & & A & & 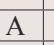 & A & A & A & A & - & - & & & $\mathrm{A}$ & A & $\mathrm{A}$ & A & & & & A & A & A & & & | & - & \\
\hline 825 & $\mathrm{AD}$ & $>30$ ans & {$[30+]$} & I & - & A & A & A & A & A & A & $\mathrm{A}$ & & A & A & A & A & A & A & A & - & - & A & A & A & A & A & A & $\begin{array}{ll}4 \\
A\end{array}$ & A $:$ & A & A & - & $\mathrm{A}$ & $\mathrm{A}$ & A & - & - & $\mathrm{A}$ \\
\hline 826 & $\mathrm{AD}$ & $20-25$ ans & {$[20-29]$} & I & - & A & A & A & A & A & B & & & C & & & A & $\mathrm{A}$ & A & A & & & - & A & A & A & $\mathrm{A}$ & & B & $\begin{array}{l}B \quad B \\
B\end{array}$ & & & & A & A & A & A & & f \\
\hline 833 & $\frac{\mathrm{IM}}{\mathrm{IM}}$ & \begin{tabular}{|l|}
$16-18$ ans \\
$15-2$ an
\end{tabular} & {$\left[\begin{array}{l}{[15-19]} \\
{[1-4]}\end{array}\right.$} & $\frac{1}{I}$ & - & A & & & - & & & & & & & & & - & A & $\mathrm{A}$ & & A & & & - & & & & & 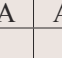 & & & & & A & A & A & A & A \\
\hline 841 & IM & $4-6$ ans & {$[1-4]$} & & & -5 & A & & & A & & $\mathrm{A}$ & & $z$ & $\pi$ & A & & & $1-$ & A & & & & B & & & & & & & & & & & & A & & & $\mathrm{B}$ \\
\hline $\begin{array}{l}842 \\
859 \\
58\end{array}$ & $\mathrm{AD}$ & $21-25$ ans & {$[20-29]$} & I & A & A & A & A & A & B & A & c & c & $\mathrm{C}$ & A & B & A & $\mathrm{A}$ & A & A & A & A & A & A & A & $\mathrm{A}$ & A & NC & $\mathrm{ON}$ & $\mathrm{ON}$ & VO N & IO & A & A & $\mathrm{A}$ & A & A & A & 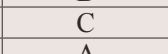 \\
\hline 858 & $\mathrm{IM}$ & \begin{tabular}{|l}
$13-18$ ans \\
$99-10$ as
\end{tabular} & {$\left[\begin{array}{l}{[15-19]} \\
{[5-9]}\end{array}\right.$} & \begin{tabular}{|l}
$\mathrm{I}$ \\
$\mathrm{I}$
\end{tabular} & $\mathrm{NO}$ & $\overline{\mathrm{A}}$ & $\bar{A}$ & A & A & B & & & & $\mathrm{A}$ & $\mathrm{A}$ & B & & A & - & A & \begin{tabular}{|l}
$\mathrm{A}$ \\
$\mathrm{A}$
\end{tabular} & NO & $\bar{A}$ & $\overline{\mathrm{A}}$ & A & A & B & A & \begin{tabular}{l|l}
$A$ \\
$A$
\end{tabular} & 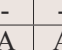 & $\mathrm{A}$ & $\overline{\mathrm{A}}$ & B & A & A & A & $\mathrm{A}$ & A & $\mathrm{F}$ \\
\hline 864 & $\mathrm{AD}$ & & {$[30+]$} & $F$ & & 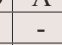 & NO & $\frac{\mathrm{A}}{\mathrm{B}}$ & \begin{tabular}{|l} 
A \\
\end{tabular} & $\frac{\mathrm{B}}{\mathrm{A}}$ & $\mathrm{NC}$ & $\mathrm{B}$ & & 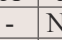 & IO & D & B & A & & NO & & NO & & & $\frac{\mathrm{A}}{\mathrm{NO}}$ & $\frac{\mathrm{A}}{\mathrm{NO}}$ & & $\frac{\mathrm{A}}{\mathrm{NC}}$ & $\begin{array}{ccc}\mathrm{A} & \mathrm{A} \\
\mathrm{O} & \mathrm{A}\end{array}$ & $\begin{array}{ll}A & t \\
A & t\end{array}$ & $\frac{\mathrm{A}}{\mathrm{A}}$ & $\frac{\mathrm{A}}{\mathrm{A}}$ & & & No & & $\frac{\mathrm{A}}{\mathrm{A}}$ & & B \\
\hline 869 & $\mathrm{AD}$ & $40-49$ ans & {$[30+]$} & $\mathrm{F}$ & - & - & - & - & - & - & - & $\pi$ & 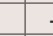 & - & - & - & -1 & -1 & -1 & - & -1 & - & - & & - & - & - & -1 & - & & & & & & - & - & & - & \\
\hline & & $15-17$ ans & & & & & & & & - & & & & - & & & & & & & & - & - & - & - & - & - & - & - & $-T_{-1}$ & - & - & - & - & - & - & - & - & \\
\hline 894 & $\mathrm{IM}$ & $10-12$ ans & {$[10-14]$} & I & - & - & - & $1-$ & - & - & - & - & 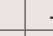 & - & $\begin{array}{lll}- & -\end{array}$ & & - & -1 & $-\theta_{1}$ & - & - & - & - & - & - & - & - & - & - & $-T_{-1}$ & - & - & - & - & -1 & - & - & 2 & \\
\hline (1) & $\mathrm{IM}$ & & [0] & 1 & - & - & - & & - & - & & & & - & & & & - & - & -5 & & - & 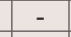 & & & - & - & & & & & & & & - & - & & & \\
\hline 000 & IN & Péri & {$[0]$} & I & - & - & 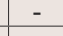 & - & - & - & - & - & - & - & - & - & - & - & - & - & - & - & - & - & - & - & - & - & - & 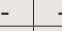 & -1 & - & - & - & - & - & -5 & - & 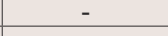 \\
\hline 900 & II & & {$[30+1]$} & $\mathrm{M}$ & - & -5 & - & - & - & - & - & - & & - & -5 & - & - & - & - & - & - & - & - & - & - & - & - & - & $\therefore$ & -3 & - & - & -5 & - & - & - & - & - & - \\
\hline 904 & $\mathrm{AD}$ & $30-59$ ans & {$[30+]$} & \begin{tabular}{|l} 
\\
\end{tabular} & - & - & - & - & - & - & - & 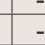 & 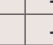 & - & - & - & - & - & - & - & - & - & - & - & - & - & - & - & - & - & - & $\begin{array}{l}- \\
-\end{array}$ & - & - & -1 & - & - & - & \\
\hline 124 & & Périnatal & {$[0]$} & 1 & & & & & & & & & & & & & & & & & & & & & & & & & & & & & & & & & & & \\
\hline & $\mathrm{AD}$ & $>40$ ans & {$[30+]$} & $F$ & NO & NO & NO & NO & NO & $\frac{\mathrm{NO}}{\mathrm{D}}$ & $\frac{N C}{N C}$ & & & $\begin{array}{c}C \\
C\end{array}$ & $\frac{10}{10}$ & NO & NO & NO & NO & NO & - & NO & A & B & A & B & B & B & \begin{tabular}{l|l}
3 & $B$ \\
\end{tabular} & & $\frac{A}{D}$ & $\frac{B}{B}$ & $\frac{B}{B}$ & $\mathrm{~A}$ & A & NO & NO & NO & \\
\hline $\begin{array}{l}1250 \\
1251\end{array}$ & $\frac{\mathrm{AD}}{\mathrm{IM}}$ & $\begin{array}{l}20-49 \text { ans } \\
15-3 \text { ans }\end{array}$ & {$[20+]$} & M & - & A & A & A & A & B & NC & & & No & NO & B & A & A & A & A & - & A & A & A & A & A & NO & NC & & & & & & A & A & A & A & A & \\
\hline & $\mathrm{AD}$ & & & \begin{tabular}{|l} 
\\
$F$
\end{tabular} & NO & & A & NO & $\overline{\mathrm{NO}}$ & NO & $\bar{A}$ & $\bar{A}$ & & & $\bar{A}$ & A & & NO & & A & & - & NO & A & NO & A & $\overline{\mathrm{A}}$ & $\overline{\mathrm{A}}$ & $A_{-}$ & $A$ & $\bar{A}$ & A & & $\mathrm{NO}$ & NO & \begin{tabular}{|l}
$\mathrm{A} O$ \\
$\mathrm{NO}$
\end{tabular} & A & NO & \\
\hline & & & {$[15-19]$} & I & A & A & A & A & A & $\mathrm{A}$ & 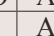 & $\frac{B}{B}$ & & $\mathrm{~B}$ & A & A & 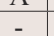 & $\mathrm{A}$ & A & $\mid \mathrm{A}$ & A & - & A & 100 & - & A & A & $\mathrm{B}$ & $\begin{array}{ll}3 \\
3\end{array}$ & $\begin{array}{ll}3 \\
B\end{array}$ & $\mathrm{~B}$ & B & A & A & A & - & $\mathrm{A}$ & - & \\
\hline & IM & $15-1$ & {$[15-19]$} & $\mathrm{I}$ & $\mathrm{A}$ & $\mathrm{A}$ & $\mathrm{A}$ & A & A & $\mathrm{A}$ & A & & & A & $\mathrm{A}$ & A & & A & A & A & A & A & $\mathrm{A}$ & $\mathrm{A}$ & $\mathrm{A}$ & A & A & $\mathrm{A}$ & & & A & & $\mathrm{A}$ & $\mathrm{A}$ & A & A & $\mathrm{A}$ & $\mathrm{A}$ & \\
\hline 131 & $\mathrm{AD}$ & 70 & {$[20+]$} & I & & - & A & $\mathrm{A}$ & A & $\mathrm{A}$ & $\mathrm{NC}$ & $\hat{N}$ & & Oo & NO & $\mathrm{A}$ & $\mathrm{A}$ & A & A & A & - & A & NO & NO & NO & NO & NO & NC & $\mathrm{O} \mathrm{NG}$ & 101 & OO & IO & OO & - & $\because$ & - & -1 & - & $\mathrm{A}$ \\
\hline 131 & & $20-4$ & {$[30+]$} & $F$ & & & & & & & & & & & & & & & & & & & & & & & - & & & & & & & & & & & & \\
\hline & IM & Périnatal & {$[0]$} & I & - & - & - & - & - & - & - & - & - & - & 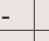 & - & - & - & - & - & - & - & - & - & - & - & - & - & - & -1. & - & - & - & - & - & - & -1 & - & \\
\hline 131 & $\frac{\mathrm{IM}}{\mathrm{AD}}$ & \begin{tabular}{|l|l|}
$16-19$ ans \\
$>20$ ans
\end{tabular} & {$[15$.} & \begin{tabular}{|l}
$\mathrm{I}$ \\
$\mathrm{I}$
\end{tabular} & - & - & - & - & - & - & - & - & & - & - & - & - & - & - & - & & - & - & - & - & - & - & - & - & - & - & - & - & - & - & - & - & - & \\
\hline 13 & & $6-9,25$ & {$[5-9]$} & \begin{tabular}{|l} 
\\
I
\end{tabular} & - & A & $\bar{A}$ & - & A & A & - & 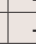 & & A & $\mathrm{A}$ & - & A & - & A & - & - & $\div$ & - & - & - & - & - & - & 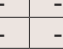 & 87 & $\bar{A}$ & - & & & - & A & - & - & A \\
\hline & IN & 8.10 & & & & & & & & & & & & & & & & - & & & & - & & & & & & & & & & & & & & & & & \\
\hline 13 & $\frac{\mathrm{Mu}}{\mathrm{ND}}$ & $12-14$ ans & {$\left[\frac{10-14]}{[30]}\right.$} & I & A & A & A & A & A & B & B & $\mathrm{F}$ & & A & NO & B & A & A & A & A & A & - & A & A & A & A & C & B & $\begin{array}{ll}3 & B \\
\end{array}$ & $\begin{array}{l}B \mathrm{~B} \\
\mathrm{~B}\end{array}$ & $\begin{array}{ll}B & 1 \\
\end{array}$ & B & $\mathrm{C}$ & - & NO & A & A & A & c \\
\hline 133 & $\frac{\mathrm{AD}}{\mathrm{IM}}$ & $\begin{array}{l}20-59 \text { ans } \\
\text { Périnatal }\end{array}$ & {$[30+$} & \begin{tabular}{|l} 
\\
\end{tabular} & - & - & - & & - & - & & & & - & & & - & - & - & - & & $\because$ & - & - & & - & - & & & & - & & & & - & - & & & \\
\hline 134 & $\mathrm{AD}$ & actina & {$[30+]$} & M & & - & & & & - & & & & & & & & - & & & & - & & & & & & & & & & & & & & & & & \\
\hline & & $14-1$ & {$[15-19]$} & I & - & - & - & - & - & - & - & -1 & - & - & - & - & - & - & - & - & - & - & - & - & - & - & - & - & - & -1 & - & - & & & - & $5-$ & - & - & \\
\hline 134 & $\mathrm{AD}$ & & & M & & - & - & 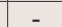 & - & & & & & & & & & - & - & - & & - & - & - & & 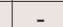 & & & & & & & & & 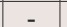 & 2 & - & & \\
\hline & IM & & $10-$ & I & - & A & A & $\mathrm{A}$ & $\mathrm{A}$ & B & B & $\mathrm{E}$ & $\mathrm{F}$ & B & B & B & A & A & A & A & & $\mathrm{A}$ & A & A & & A & C & B & B & B & B & B & $\mathrm{C}$ & A & A & A & A & & \\
\hline 135 & IM & & {$[10-$} & I & - & A & C & A & A & C & B & & & & B & C & A & A & $\mathrm{C}$ & A & A & & A & C & A & A & C & C & B & $\begin{array}{l}B \\
B\end{array}$ & B & $\begin{array}{c}\mathrm{C} \\
\mathrm{C}\end{array}$ & C & A & A & C & A & A & C \\
\hline & $\mathrm{IM}$ & & {$[5-9$,} & 1 & - & - & - & - & - & - & - & - & -1 & - & - & - & - & - & - & - & - & - & - & - & - & - & - & - & - & & 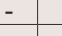 & - & - & -1 & - & - & - & & \\
\hline 136 & $A D$ & & & $M$ & - & $\therefore$ & & & & - & & & & & & & & - & & - & & & & & & & & & & & & & & & & & & & \\
\hline 136 & $n$ & & {$[5$} & $M$ & - & - & A & & $\mathrm{A}$ & & & & & $\mathrm{A}$ & & & & & A & & & & B & & & $\mathrm{B}$ & & & & & & & & & & $A$ & $\mathrm{~B}$ & & \\
\hline & $\mathrm{AD}$ & & {$[20+]$} & $\mathrm{M}$ & $\mathrm{A}$ & $\mathrm{A}$ & NO & $\mathrm{A}$ & A & A & $\mathrm{A}$ & 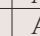 & & $\mathrm{A}$ & $\mathrm{A}$ & $\mathrm{A}$ & 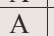 & A & NO & A & $\mathrm{A}$ & $\mathrm{A}$ & NO & $\mathrm{C}$ & $\mathrm{A}$ & $\mathrm{A}$ & $\mathrm{A}$ & $\mathrm{A}$ & A & & $\mathrm{A}$ & A & $\bar{A}$ & $\mathrm{~A}$ & A & $\mathrm{C}$ & $\mathrm{A}$ & & \\
\hline 13 & & & 5 & & & & & & & & & & & & & & & & & & & & & & & & & & & & & & & & & & & & \\
\hline
\end{tabular}

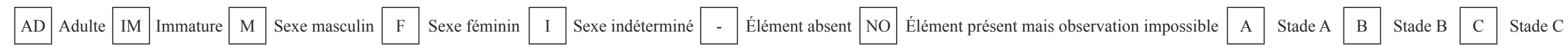


Annexe 2B. Données paléobiologiques individuelles pour l'échantillon de la Cathédrale (1/5) (téléchargeable sur https://journals.openedition.org/bmsap/7739?file=1)

\begin{tabular}{|c|c|c|c|c|c|c|c|c|c|c|c|c|c|c|c|c|c|c|c|c|c|c|c|c|c|c|c|c|c|c|c|c|c|c|c|c|c|c|c|}
\hline & & Age & & & & Нур & opla & sie $m$ & axil & laire & droit & & & Нуро & plasi & ie ma & axilla & aire ge & auche & & & Нуро & oplas & ie $\mathrm{m}$ & andil & bule & droite & & & Hуро & plasi & ie ma & andib & bule g & gauch & & & & Cribra orbitalia \\
\hline & Categorie & Estimation & Classe & & M3 & M2 & M1 & P2 & P1 & C & 12 & I1 & I1 & 12 & C & P1 & P2 & M1 & M2 & M3 & M3 & M2 & M1 & P2 & P1 & C & 12 & I1 & I1 & I2 & C & P1 & P2 & M1 & 1 M2 & M3 & individu & Orbite droite & Orbite gauche \\
\hline 1 & $\mathrm{AD}$ & $>50$ ans & {$[30+]$} & M? & - & - & - & - & - & - & - & - & - & - & - & - & - & - & - & - & - & - & - & - & - & - & - & - & - & - & - & - & - & - & - & - & - & & - \\
\hline 2 & $\mathrm{AD}$ & $30-6$ & {$[30+]$} & M? & - & & & -5 & -5 & & & - & & & & & 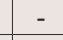 & & & - & - & & 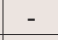 & 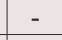 & - & & & & 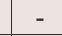 & & & & - & & & & & & \\
\hline & $\mathrm{AD}$ & 2 & {$[20+]$} & M? & - & A & A & A & B & B & B & B & B & B & B & A & A & A & A & 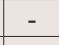 & - & A & A & A & A & B & C & NO & E & B & C & A & A & A & A & & $\mathrm{C}$ & Pas de lésion & Pas de lésion \\
\hline 4 & $\mathrm{AD}$ & $40-60$ ans & {$[30+]$} & M? & - & - & - & - & - & -5 & & - & - & & & & - & - & & - & - & - & - & - & - & & & & - & & - & - & - & - & & - & & as de lésion & Pas de lésion \\
\hline 5 & $\mathrm{D}$ & $>50$ ans & {$[30+]$} & $\mathrm{M}$ & - & A & A & NO & $\mathrm{NO}$ & B & $\mathrm{NO}$ & - & - & B & B & B & A & A & B & - & A & A & A & A & A & $\mathrm{NC}$ & NO & $\mathrm{NO}$ & E & $\mathrm{NO}$ & C & A & - & A & B & & $\mathrm{C}$ & Pas de lésion & Présence \\
\hline 6 & D & $25-40$ ans & {$[20+]$} & M? & - & -1 & - & $\begin{array}{lll}n_{1} & \\
\end{array}$ & - & - & - & - & - & - & & - & - & - & & $\mid-$ & - & - & & - & - & - & - & - & - & - & - & - & - & - & - & - & - & & \\
\hline 7 & $\mathrm{AD}$ & $20-30$ ans & {$[20-29]$} & M? & - & -1 & - & - & - & - & - & - & - & - & -1 & - & - & - & - & - & - & - & - & - & - & - & - & - & - & - & - & - & - & - & - & - & - & & - \\
\hline 8 & $\mathrm{D}$ & $>20$ ans & {$[20+]$} & I & - & - & - & - & - & - & - & - & - & - & - & - & - & - & - & - & - & - & - & - & - & - & - & - & - & - & - & - & - & - & 2 & - & 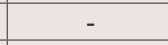 & - & - \\
\hline 9 & $\mathrm{AD}$ & $40-60$ ans & {$[30+]$} & M? & - & - & - & - & - & - & - & - & - & - & - & - & - & - & - & - & B & - & - & - & - & - & - & - & - & - & - & NO & A & B & B & A & B & 2 & - \\
\hline 10 & $\mathrm{AD}$ & $40-60$ ans & {$[30+]$} & M & - & C & C & NO & B & & - & - & - & & 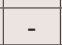 & C & B & & & - & - & A & & B & A & C & - & - & - & 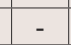 & 5 & 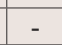 & & - & B & & $S_{1}$ & Pas de lésion & Pas de lésion \\
\hline 11 & $\mathrm{AD}$ & 20 & {$[20+$} & I & - & - & - & - & - & - & - & - & - & - & - & - & - & - & - & - & - & - & - & 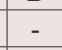 & - & - & - & - & - & - & - & - & - & - & - & - & & is de lésion & as de lésion \\
\hline 12 & $A$ & $>2$ & {$[20+]$} & I & - & - & - & - & - & - & - & - & - & - & - & - & - & - & - & - & - & - & - & - & - & - & - & - & - & - & - & - & - & - & - & - & & & \\
\hline 13 & $\mathrm{AD}$ & $30-50$ & {$[30+]$} & M? & - & - & - & - & - & - & - & - & - & - & -5 & - & - & - & - & - & - & - & - & - & - & - & - & - & - & - & - & - & - & - & - & & & & \\
\hline 15 & $\mathrm{AD}$ & $40-60$ ans & {$[30+]$} & M & A & A & & & $\mathrm{NO}$ & $\mathrm{NC}$ & & - & - & - & B & - & B & - & & B & B & - & B & NO & NO & $\mathrm{NC}$ & NO & - & - & $\mathrm{NO}$ & NO & NO & A & NO & $\mathrm{O}$ & A & B & Pas de lésion & Pas de lésion \\
\hline 16 & $\mathrm{AD}$ & $40-60$ ans & {$[30+]$} & $\mathrm{M}$ & & - & - & - & - & - & & - & - & - & - & - & - & - & - & & & - & & - & - & - & - & - & - & - & - & - & - & - & & & & & \\
\hline 20 & $\mathrm{AD}$ & $>60$ ans & {$[30+]$} & I & - & - & - & & - & - & - & - & - & - & - & - & - & - & - & - & - & - & - & - & - & - & - & - & - & - & - & - & - & - & - & - & - & Pas de lésion & Pas de lésion \\
\hline 23 & $\mathrm{AD}$ & $20-30$ ans & [20-29] & $\mathrm{M}$ & - & B & NO & NO & B & B & A & A & B & A & B & B & A & NO & B & - & - & B & B & B & A & C & NO & NO & - & NO & B & A & - & $\mathrm{C}$ & $\mathrm{C}$ & 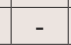 & $\mathrm{C}$ & & \\
\hline 24 & $\mathrm{AD}$ & $30-50$ ans & {$[30+]$} & I & - & 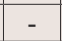 & - & - & - & & - & - & NO & $\mathrm{NO}$ & $\mathrm{C}$ & - & - & - & - & - & - & -5 & - & - & - & - & - & - & - & - & B & A & - & - & - & - & $\mathrm{s}$ & as de lésion & Pas de lésion \\
\hline 25 & $\mathrm{AD}$ & $40-60$ ans & {$[30+]$} & M & - & - & - & - & - & - & - & - & - & - & 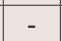 & - & - & - & - & - & - & - & - & - & - & - & - & - & - & - & $x^{2}$ & - & - & - & - & - & & & \\
\hline 26 & $\mathrm{AD}$ & $>20$ ans & {$[20+]$} & I & - & - & - & - & - & - & 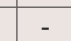 & - & - & - & - & - & - & - & - & - & - & - & - & - & - & - & - & - & - & - & - & -- & - & - & 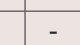 & - & 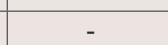 & & \\
\hline 27 & F & $20-$ & {$[20-29]$} & $\mathrm{F}$ & - & - & - & - & - & - & - & - & - & - & - & - & - & - & 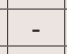 & - & - & - & & - & - & - & - & - & - & - & -5 & - & - & - & & - & & & \\
\hline 28 & & & {$[30+]$} & M? & - & - & & - & - & - & & - & - & & & & - & - & & & - & - & & - & - & - & - & - & - & - & & - & - & - & - & - & & de lésion & de lésion \\
\hline 29 & $\mathrm{AD}$ & $30-50$ ans & {$[30+]$} & $\mathrm{F}$ & - & - & - & - & - & - & - & - & - & - & - & - & - & - & - & - & - & - & - & - & - & - & - & - & - & - & - & - & - & - & - & - & - & & \\
\hline 30 & $\mathrm{IM}$ & $16-20$ ans & [15-19] & I & - & - & - & - & - & - & - & - & - & - & - & - & - & - & - & - & - & - & - & - & - & - & - & - & - & - & - & - & - & - & - & - & - & & - \\
\hline 31 & $\mathrm{AD}$ & $30-50$ ans & {$[30+]$} & $\mathrm{F}$ & - & - & & & - & 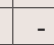 & & - & - & 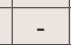 & 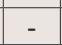 & - & - & $\mathrm{C}$ & & & - & - & & - & - & - & - & - & - & - & & NO & - & - & - & 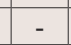 & c & Pas de lésion & Pas de lésion \\
\hline 32 & $\mathrm{AD}$ & $20-50$ ans & {$[20+]$} & $\mathrm{M}$ & - & - & - & - & - & - & - & - & - & - & - & - & - & - & - & - & - & - & - & - & - & - & - & - & - & - & - & - & - & - & - & - & - & & \\
\hline 33 & $\mathrm{AD}$ & $20-40$ ans & {$[20+]$} & $\mathrm{F}$ & - & - & - & - & - & - & - & - & - & - & - & - & - & - & - & - & - & - & - & - & - & - & - & - & - & - & - & - & - & - & - & - & - & & - \\
\hline 34 & $\mathrm{AD}$ & $30-50$ ans & {$[30+]$} & I & - & - & - & - & - & - & - & - & - & - & - & - & - & - & - & - & - & - & - & - & - & - & - & - & - & - & - & - & - & - & - & - & & & - \\
\hline 35 & & $>2$ & {$[20+]$} & I & - & - & - & - & - & - & - & - & - & - & - & - & - & - & - & - & - & - & - & - & - & - & - & - & - & - & - & - & - & - & - & - & . & - & - \\
\hline 36 & & & 120 & I & & - & & - & - & - & - & - & - & - & - & - & - & - & - & - & - & - & - & - & - & - & - & - & - & - & - & - & - & - & - & - & & & \\
\hline 37 & & 17. & $\frac{[20+1}{[15-19]}$ & 1 & & - & & & - & - & & & - & - & & - & - & - & - & & - & - & & - & - & - & - & - & - & - & - & - & - & 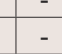 & & & & & \\
\hline 38 & & & {$[20-29]$} & M & A & - & - & A & A & A & A & A & A & A & NO & A & - & - & A & A & & A & A & A & A & A & A & A & A & A & A & A & A & A & A & A & & ésion & s de lésic \\
\hline 39 & $\mathrm{AL}$ & $40-60$ ans & {$[30+]$} & M? & A & A & A & A & A & A & A & - & - & A & A & - & A & A & A & A & - & B & $\mathrm{A}$ & $\mathrm{A}$ & A & $\mathrm{A}$ & $\mathrm{A}$ & A & $\mathrm{A}$ & $\mathrm{A}$ & $\mathrm{A}$ & $\mathrm{A}$ & A & $\mathrm{A}$ & B & & D & is de lésion & \\
\hline 40 & $\mathrm{AL}$ & $40-60$ ans & {$[30+]$} & M & - & - & & - & - & - & - & - & - & - & - & - & - & - & - & - & - & - & - & - & - & - & - & - & - & - & - & - & - & - & $\sigma^{2}$ & - & 2 & & \\
\hline 41 & $\mathrm{AD}$ & ans & {$[30+]$} & M & A & & A & A & A & A & A & B & A & A & A & A & A & - & A & A & A & A & & A & A & $\mathrm{A}$ & A & A & A & A & A & A & - & - & A & & B & Pas de lésion & Pas de lésion \\
\hline 42 & $\mathrm{AL}$ & $>$ & {$[20+]$} & I & & - & & & & & & & & & & & & -2 & & & & & & - & - & - & & - & - & - & & - & - & - & & & & & \\
\hline 43 & & 20 & {$[2$} & I & A & A & A & A & A & A & A & B & B & A & A & A & A & A & A & A & & - & & - & - & - & - & - & - & - & - & - & - & - & & & $\mathrm{H}$ & & as de lésion \\
\hline & & & {$[15-19]$} & I & A & A & A & A & $\mathrm{A}$ & A & A & A & $\mathrm{A}$ & A & A & A & A & A & A & A & $\mathrm{A}$ & A & A & A & A & A & A & A & $\mathrm{A}$ & $\mathrm{A}$ & A & A & A & $\mathrm{A}$ & A & A & & Pas de lésion & Pas de lésion \\
\hline 45 & & & {$[5$} & I & & - & & - & - & - & - & - & NO & NO & A & A & $\mathrm{A}$ & NO & A & - & - & - & & - & - & - & - & - & $\because$ & - & - & - & - & - & - & - & & & \\
\hline 47 & $\mathrm{AD}$ & $30-50$ ans & {$[30+]$} & M? & +2 & B & B & A & A & B & NO & NO & B & B & $\mathrm{B}$ & A & $\mathrm{A}$ & B & B & 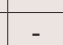 & - & A & A & A & A & - & - & - & - & $\mathrm{C}$ & $\mathrm{C}$ & - & A & NO & $\begin{array}{lll}0 & \mathrm{~A} \\
\end{array}$ & & ( & résence & Présence \\
\hline 48 & $\mathrm{AL}$ & & {$[20-29]$} & M? & A & A & A & A & $\mathrm{A}$ & A & A & A & NO & NO & A & A & $\mathrm{A}$ & NO & A & A & A & $\mathrm{A}$ & $\mathrm{A}$ & B & B & A & NO & NO & NO & NO & B & A & A & A & $\mathrm{A}$ & B & & & \\
\hline 51 & $\mathrm{AL}$ & & {$[20-$} & I & & - & & - & 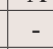 & 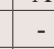 & 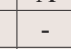 & $2 \times$ & - & 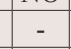 & & 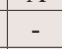 & - & 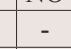 & & 42 & 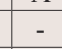 & & & & D & 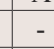 & & 4 & - & - & & & 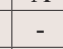 & (2) & & & & & \\
\hline 52 & $\mathrm{AL}$ & $25-40$ & {$[20$} & I & 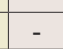 & 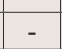 & & & -5 & & & & & & & & - & & & & A & A & B & & - & & & & - & & & & - & - & NO & $\mathrm{NO}$ & & $\mathrm{sdc}$ & Pas de le \\
\hline 53 & $\mathrm{IM}$ & & {$[15-19]$} & M? & A & A & B & A & $\mathrm{A}$ & A & A & & & - & B & A & A & NO & $\mathrm{C}$ & A & A & $\mathrm{A}$ & $\mathrm{C}$ & A & A & B & A & NO & A & A & B & B & B & $\mathrm{C}$ & No & A & & NO & \\
\hline 54 & $\mathrm{AL}$ & 25 & {$[20$} & I & & NO & A & & A & B & & & B & & $\mathrm{C}$ & A & C & NO & A & NO & & & & & & - & & & & & & & - & & & & $c$ & Pas de lésion & Pas de lésion \\
\hline 55 & & & {$[2 \pi$} & I & & -1 & & & & & & & & & & & & & & & & & & & & & & & & & & & & & & & & & \\
\hline 56 & & & {$[36$} & M & & - & - & - & - & & & - & & & - & - & - & & - & - & - & - & & - & - & - & - & - & - & - & - & - & - & - & - & - & & - & - \\
\hline & $\mathrm{AD}$ & $20-$ & {$[20-29]$} & $\mathrm{F}$ & A & A & B & A & $\mathrm{A}$ & A & B & $\mathrm{C}$ & $\mathrm{C}$ & B & B & A & A & A & A & A & - & & 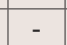 & 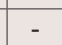 & - & & - & 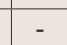 & - & 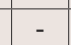 & -5 & 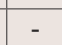 & - & & - & & $\mathrm{c}$ & & \\
\hline & & $>$ & {$[20$} & I & & $\because \pi$ & & $\because$ & - & & & 年 & - & & & $\because$ & - & 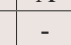 & Net & (2) & - & - & & - & - & - & - & - & - & - & - & - & - & - & - & - & & - & - \\
\hline 60 & $\mathrm{AD}$ & $>2$ & {$[20+]$} & I & & - & & - & 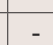 & & & - & - & - & & 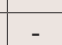 & - & 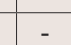 & & 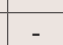 & - & - & & 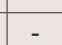 & - & +2 & - & - & - & - & - & - & 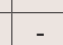 & - & & -5 & - & & \\
\hline 61 & A & $30-60$ & {$[30$} & $\mathrm{F}$ & & & & & - & & & - & & - & & - & - & - & 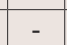 & - & - & - & & - & - & - & 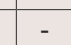 & - & -5 & - & 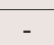 & - & - & - & 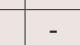 & - & & & -7 \\
\hline 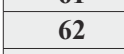 & $x^{2}$ & $-x_{2}$ & {$[20-29$} & 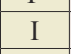 & & -5 & & - & +5 & 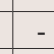 & & 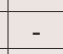 & tat & & & 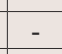 & - & & -5 & 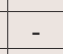 & - & & & & & & & & & & & & -5 & -5 & & & & & \\
\hline 63 & & & {$[26$} & 1 & & & & 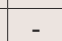 & - & - & & 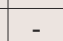 & - & & & 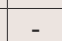 & - & - & 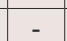 & - & - & & & $\mathrm{A}$ & A & B & B & B & B & B & B & A & A & 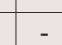 & & & & Présenc & résen \\
\hline 0 & & & {$[3$} & I & & & & & & & & & & & & & & & & & - & & & - & - & 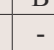 & & & $D$ & & & & - & & & & & & \\
\hline 6 & In & & {$[1$.} & I & & & & & & & & & & & & & & - & & & & - & & & & & & & & - & - & & - & - & & & & & \\
\hline 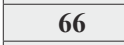 & $\mathrm{AD}$ & $>20$ ans & {$[20+]$} & $I_{1}$ & & - & & & & & & & 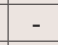 & & & & $-z_{2}$ & 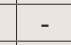 & & $-z_{-1}$ & $-z_{-1}$ & 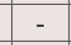 & & $-y_{-1}$ & $-z_{-1}$ & & & $-y_{-1}$ & - & 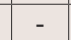 & - & - & - & - & - & & & & \\
\hline 67 & $\mathrm{AD}$ & $>20$ ans & {$[20+]$} & $\mathrm{I}$ & - & - & & & - & - & & & $1-$ & - & & $1-$ & $1-$ & - & & & - & - & & & $1-$ & - & & & $1-$ & - & & & & - & & & & & \\
\hline
\end{tabular}

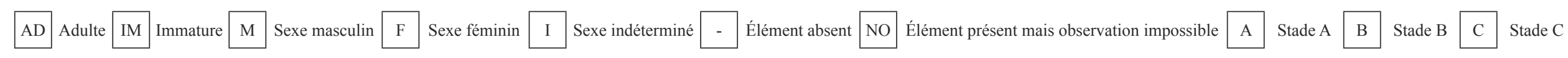


Annexe 2B. Données paléobiologiques individuelles pour l'échantillon de la Cathédrale (2/5)

\begin{tabular}{|c|c|c|c|c|c|c|c|c|c|c|c|c|c|c|c|c|c|c|c|c|c|c|c|c|c|c|c|c|c|c|c|c|c|c|c|c|c|c|c|c|}
\hline & & Age & & & & & oplas & sie m & axil & laire & droi & & & & plasi & ie ma & axilla & aire ga & auche & & & Нуро & plasi & ie ma & andib & bule d & droite & & & Нурою & plasic & ie ma & andib & bule g & gaucl & & & & Cribra orbitalia & \\
\hline entre & Categorie & Estimation & Classe & & M3 & M2 & M1 & P2 & P1 & $\mathrm{C}$ & 12 & I1 & I1 & 12 & C & P1 & P2 & M1 & M2 & M3 & M3 & M2 & M1 & P2 & P1 & C & 12 & I1 & I1 & 12 & C & P1 & P2 & M1 & $1 \mathrm{M} 2$ & \begin{tabular}{|l|l|}
2 M3 \\
\end{tabular} & individu & Orbite droite & Orbite gauche & Individu \\
\hline 68 & $\mathrm{AD}$ & $20-30$ ans & 20-29] & $\mathrm{M}$ & - & - & -7 & - & - & - & - & - & - & 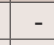 & $1-$ & - & - & - & - & - & - & -7 & - & - & - & - & - & - & - & - & - & - & - & - & - & - & 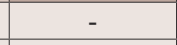 & & & - \\
\hline 69 & IM & 14-17 ans & {$[10-14]$} & $F ?$ & & - & & - & - & - & & - & - & - & - & - & & - & & - & - & 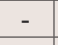 & - & - & - & & - & - & - & - & - & - & - & - & - & - & - & - & - & - \\
\hline 70 & $\mathrm{AD}$ & $>20$ & {$[20+]$} & 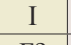 & - & - & - & - & - & 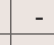 & - & - & - & - & - & - & - & - & - & - & - & - & - & - & - & - & - & - & - & - & - & - & - & - & - & - & - & - & - & - \\
\hline 71 & D & $20-4$ & {$[20$} & $\mathrm{F}$ ? & - & - & - & - & - & - & - & - & - & -5 & - & - & - & - & - & - & - & - & - & - & - & - & - & - & - & - & - & - & - & - & - & - & - & - & & - \\
\hline 72 & $\mathrm{AD}$ & $>20$ ans & {$[20+]$} & I & - & $-a_{-1}$ & & - & - & - & - & - & - & - & - & - & -3 & - & - & - & - & - & - & - & - & - & - & - & - & - & -5 & - & - & - & - & - & - & - & - & - \\
\hline 73 & $\mathrm{IM}$ & 6-8 ans & {$[5-9]$} & $\mathrm{I}$ & - & - & - & - & - & - & - & - & - & - & - & - & - & - & - & - & -1 & - & - & - & - & - & - & - & - & - & - & - & - & - & - & - & - & - & - & - \\
\hline 74 & IM & $2-4$ ans & {$[1-4]$} & I & - & - & - & - & - & - & - & - & - & - & - & - & - & - & - & - & & - & - & - & & - & - & - & & - & - & - & - & - & - & - & - & - & - & -2 \\
\hline 77 & $\mathrm{IM}$ & 0-6 mois & {$[0]$} & I & - & $-{ }_{-1}$ & - & - & - & - & - & - & - & - & - & - & - & - & - & - & - & - & - & - & - & - & - & - & - & - & $-e^{-}$ & - & - & - & - & - & - & - & - & - \\
\hline 78 & 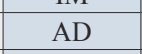 & $>20$ ans & {$[20+]$} & $\mathrm{I}$ & - & - & - & - & - & - & - & - & - & - & - & - & - & - & - & - & - & - & - & - & - & - & - & - & - & - & - & - & - & - & - & - & - & -5 & -2 & -5 \\
\hline 79 & $\mathrm{AD}$ & $>20$ ans & {$[20+$} & I & - & - & -1 & - & - & - & - & - & - & 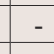 & - & - & - & - & - & - & - & - & - & - & - & - & - & - & - & - & - & - & - & 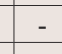 & -2 & - & - & - & - & - \\
\hline 80 & A & $>2$ & {$[20+$} & $\mathrm{I}$ & & +4 & -4 & - & - & - & - & - & - & & & - & & & & - & - & -4 & - & - & - & - & - & - & & & & - & & & & - & & & & \\
\hline 82 & $\mathrm{AD}$ & $>40$ & {$[30+]$} & I & - & - & - & - & - & - & - & - & A & - & A & A & A & A & B & - & - & - & - & - & - & - & - & - & A & A & A & A & A & NO & A & - & B & - & Pas de lésion & as de lésion \\
\hline & IM & $9-12$ ans & {$[5-9]$} & I & - & - & - & - & - & - & - & - & - & - & - & - & - & - & - & - & - & - & - & - & - & - & - & - & - & - & - & - & - & - & - & - & - & - & & \\
\hline 84 & IM & $14-16$ ans & {$[10-14]$} & I & - & - & - & - & - & - & - & - & - & 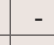 & - & - & - & - & - & - & - & - & - & - & - & - & - & - & - & - & - & - & - & 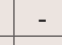 & - & - & - & - & & \\
\hline 85 & $\mathrm{IM}$ & \begin{tabular}{|l|}
$0-6$ mois \\
\end{tabular} & {$[0]$} & I & & & & & & & & - & & & & & & & & & & & & & & & & & & & & & & & & & & - & & - \\
\hline 86 & $\mathrm{AD}$ & $>60$ ans & {$[30+]$} & $\mathrm{M}$ & - & B & A & $\mathrm{A}$ & A & A & $\mathrm{NC}$ & - & - & - & - & - & NO & NO & NO & - & B & NO & NO & NO & NO & NO & NO & NO & A & NO & NO & NO & A & NO & $\mathrm{NO}$ & NO & B & - & - & - \\
\hline 87 & $\mathrm{AD}$ & $30-60$ ans & {$[30+]$} & I & - & & & - & & & & - & 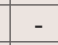 & - & - & NO & 2 & - & - & - & A & A & - & NO & NO & A & A & A & A & A & A & NO & NO & NO & $\mathrm{A}$ & B & B & NO & Pas de lésion & Pas de lésion \\
\hline 88 & IM & 14-16 ans & {$[10-14]$} & I & - & - & - & - & - & - & - & - & - & - & - & - & - & - & - & - & - & - & - & - & - & - & - & - & - & - & - & - & - & 2 & - & 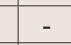 & & & - & - \\
\hline 89 & $\mathrm{AD}$ & 40-60 ans & {$[30+]$} & $\mathrm{F}$ & - & - & - & - & - & 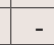 & - & - & - & 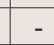 & - & - & - & - & - & - & - & - & - & - & - & - & - & - & - & - & - & - & - & - & - & - & - & - & - & 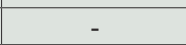 \\
\hline 90 & $\mathrm{AD}$ & $20-30$ ans & {$[20-29]$} & $\mathrm{F}$ & 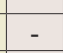 & 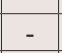 & -4 & - & - & - & - & - & - & - & - & - & - & - & - & - & - & - & - & - & - & - & - & - & - & - & - & - & - & -5 & - & - & - & - & & \\
\hline 91 & & & {$[5-9]$} & 1 & & - & - & - & - & - & - & - & - & - & - & - & - & -3 & - & - & - & & - & - & - & - & - & - & - & - & - & - & - & - & - & - & & - & & \\
\hline 92 & IM & $1-2$ & {$[1-4]$} & I & - & - & 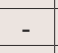 & - & 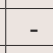 & & - & - & - & & & - & - & & & - & - & & & - & - & & & - & & & & - & - & & & - & & & de 1 & de lés \\
\hline 93 & $\mathrm{AD}$ & $40-60$ ans & {$[30+]$} & $\mathrm{M}$ & & & NO & NO & $\mathrm{NO}$ & NO & $\mathrm{A}$ & B & $\mathrm{A}$ & $\mathrm{A}$ & NO & NO & NO & NO & NO & - & - & - & NO & A & NO & NO & NO & NO & NO & NO & NO & A & A & - & NO & $5-$ & B & Pas de lésion & Pas de lésion & \begin{tabular}{|l|} 
Pas de lésion \\
\end{tabular} \\
\hline 94 & $\mathrm{AD}$ & $30-50$ ans & {$[30+]$} & I & A & NO & NO & $E$ & - & - & & - & - & A & NO & NO & NO & NO & NO & A & B & A & NO & A & A & B & 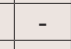 & - & - & - & B & A & A & NO & $\mathrm{A}$ & $\mathrm{A}$ & B & & & \\
\hline 95 & $\mathrm{AD}$ & $>30$ ans & {$[30+]$} & M? & - & - & - & - & - & - & - & - & - & - & - & - & - & - & - & - & - & - & - & - & - & - & - & - & - & - & - & - & - & - & - & - & & Pas de lésion & - & Pas de lésion \\
\hline 96 & $\mathrm{IM}$ & 18-22 ans & {$[15-19]$} & $\mathrm{M}$ & - & - & - & - & - & - & - & - & - & - & - & - & - & - & - & - & A & A & A & A & B & C & NO & A & NO & NO & C & B & A & $\mathrm{A}$ & $\mathrm{A}$ & - & $\mathrm{C}$ & & - & \\
\hline 97 & $\mathrm{AD}$ & $>20$ ans & {$[20+]$} & I & - & - & - & - & - & - & - & - & - & - & - & - & - & - & - & - & & & & - & & - & $2=$ & - & 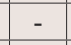 & - & - & - & & & & - & & & & - \\
\hline 98 & $\mathrm{AI}$ & $30-60$ ans & {$[30+]$} & I & - & - & - & - & - & - & - & - & - & - & - & - & - & - & - & - & - & - & - & - & - & - & - & - & - & - & - & - & - & - & - & - & - & - & - & - \\
\hline 99 & & 20 & {$[20+]$} & & - & & - & - & - & - & - & - & - & - & - & - & - & - & - & - & - & - & - & - & - & - & - & - & - & - & - & - & - & 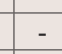 & - & - & - & & & \\
\hline 100 & & & [0] & I & & & & - & - & - & & - & - & & & - & - & & - & - & - & & & - & - & - & - & - & - & - & - & - & - & & & - & - & s de lésion & Pas de lésion & as de lésion \\
\hline 101 & & & {$[20-29]$} & $\mathrm{M}$ & A & A & NO & A & A & B & A & A & A & A & A & NO & A & NO & NO & A & - & A & A & A & A & A & A & NO & NO & - & A & A & A & & A & - & B & as de lésion & & s de lésion \\
\hline 102 & IM & 3-9 mois & {$[0]$} & I & - & - & & - & -2 & t & & - & 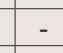 & 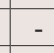 & & - & 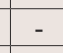 & - & - & - & - & & & - & & & & - & - & - & & - & & & & - & - & & Pas de lésion & as de lésion \\
\hline 103 & $\mathrm{Al}$ & $30-50$ ans & {$[30+]$} & M? & - & \begin{tabular}{|l|l|}
- & \\
\end{tabular} & $-{ }_{-1}$ & - & - & - & - & - & - & - & - & - & - & - & - & - & - & - & - & - & - & - & - & - & - & - & - & - & - & - & - & - & - & - & 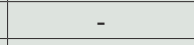 & - \\
\hline 104 & $\mathrm{AD}$ & $30-$ & {$[30+]$} & I & - & & & & & - & - & - & - & & & & & - & & - & - & & - & - & - & - & - & - & - & - & - & - & - & - & - & - & & & & \\
\hline 105 & $\mathrm{AD}$ & $30-60$ ans & {$[30+]$} & I & & A & A & A & A & - & - & - & - & A & A & A & A & - & - & - & - & - & - & - & - & - & - & - & - & - & $-{ }_{-1}$ & - & - & - & - & - & A & Pas de lésion & Pas de lésion & Pas de lésion \\
\hline 106 & $\mathrm{AD}$ & $20-25$ ans & [20-29] & $\mathrm{F}$ ? & {$[20-$} & A & A & A & A & A & A & - & B & A & A & A & A & A & A & A & A & A & A & A & A & A & B & $\mathrm{C}$ & $\mathrm{C}$ & A & в & A & A & A & A & - & $\mathrm{C}$ & Pas de lésion & Pas de lésion & Pas de lésion \\
\hline 108 & $\mathrm{AD}$ & $>20$ ans & {$[20+]$} & $\mathrm{I}$ & & 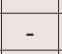 & - & - & - & - & - & - & - & - & - & - & - & - & - & - & - & - & - & - & - & - & - & - & - & - & -1 & - & - & - & - & - & - & - & 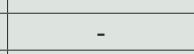 & - \\
\hline 109 & $\mathrm{AI}$ & $30-50$ ar & {$[3$} & M & - & & - & - & - & - & - & - & - & - & - & - & - & - & - & - & - & - & - & - & - & - & - & - & - & - & - & - & - & - & - & - & 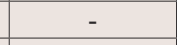 & - & - & - \\
\hline 110 & IN & 17 & {$[15$.} & $\mathrm{F}$ & & & & & & & & & & & & & & & & & & & & & - & & & & & & & - & - & & & - & - & & & - \\
\hline 111 & & & {$[36$} & $\mathrm{F}$ & - & $-{ }_{-1}$ & - & - & - & - & - & - & 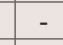 & - & - & - & - & - & - & - & - & - & - & - & - & - & - & - & - & - & - & - & - & - & - & - & - & - & - & - \\
\hline 112 & & & {$[20-$} & $\mathrm{M}$ & - & - & & - & - & - & - & - & - & - & & - & - & & - & - & & & & - & - & - & & - & & & - & - & - & & & & & & & \\
\hline 113 & & & {$[30$} & $1 \mathrm{~N}$ & & & NO & NO & A & & & - & - & & NO & A & NO & NO & & - & NO & NO & NO & NO & NO & NO & NO & NO & NO & NO & A & A & $\mathrm{NO}$ & NO & $3 \mathrm{NO}$ & $\mathrm{NO}$ & A & s de lésion & Pas de lésion & as de lésion \\
\hline 114 & $\mathrm{Al}$ & $30-$ & {$[30+]$} & $\mathrm{M}$ & $\mathrm{C}$ & C & NO & NO & NO & NO & $\mathrm{NC}$ & NO & - & $\mathrm{NO}$ & 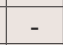 & NO & NO & B & B & C & NO & B & B & B & B & NO & NO & NO & NO & NO & C & C & A & $\mathrm{A}$ & NO & $\mathrm{NO}$ & $\mathrm{C}$ & & Pas de lésion & Pas de lésion \\
\hline 115 & $\mathrm{AD}$ & 25 & {$[20+]$} & $\mathrm{M}$ & & & & 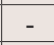 & & & & & & & & & & & & & & & & & & & & & & & & & & & & & & & & \\
\hline 116 & $\mathrm{Al}$ & & {$[30+$} & 5 & - & & - & - & - & - & - & - & B & NO & B & - & A & & 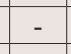 & - & - & & - & - & - & & - & - & - & & - & NO & - & - & - & - & B & s de lésion & Pas de lésion & as de lésic \\
\hline 117 & & & {$[30-$} & & & & & - & -5 & & & - & & & & - & & & & & & & & 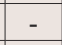 & & & & - & & & & & & & & & & & & \\
\hline 11 & $\mathrm{AI}$ & & {$[30$} & & & - & & - & - & - & - & - & - & - & & - & - & & - & - & - & & & - & - & & & - & - & & - & 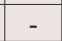 & - & & & - & & & & \\
\hline 11 & $\mathrm{~A}$ & & {$[30$} & M? & $\mathrm{C}$ & C & B & A & A & $\mathrm{A}$ & A & $\mathrm{A}$ & $\mathrm{A}$ & B & NO & A & A & NO & C & NO & C & C & B & A & A & NO & NO & NO & NO & NO & NO & A & A & NO & $\mathrm{NO}$ & $\mathrm{NO}$ & 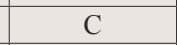 & de lésion & is de lésion & as de lésion \\
\hline 120 & $\mathrm{Al}$ & & {$[30$} & $\mathrm{I}$ & & & & & & & & & & & & & & & & & A & A & & A & A & B & & & NO & NO & B & A & B & NO & A & A & B & & & \\
\hline 12 & IN & $11-$ & {$[10-1$} & I & - & - & - & - & & & & - & & - & - & - & - & - & 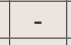 & - & - & 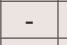 & 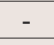 & - & 2 & 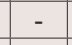 & - & - & - & - & 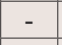 & - & - & - & & & 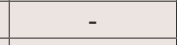 & - & - & - \\
\hline 12. & $\mathrm{~A}$ & $30-$ & {$[30+$} & $\mathrm{F}$ & & & & & & & & - & - & & & - & & - & - & - & - & & & - & & & - & 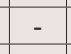 & - & - & & - & & - & & & & & & 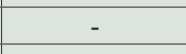 \\
\hline 124 & $\mathrm{AD}$ & $30-60 \mathrm{a}$ & {$[30+]$} & M? & A & B & $\mathrm{A}$ & A & A & A & & - & - & - & & - & - & - & - & - & - & - & -5 & - & 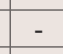 & - & - & - & - & - & - & - & - & 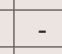 & - & - & B & & & - \\
\hline 12 & IN & $7-6$ & {$[5-9]$} & $\mathrm{I}$ & - & & A & - & - & 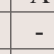 & A & $\mathrm{A}$ & A & A & - & - & - & A & - & - & - & 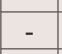 & A & 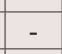 & - & - & A & $\mathrm{A}$ & A & A & t & - & - & $\mathrm{A}$ & - & - & A & Présence & Présence & Présence \\
\hline 126 & & $30-50$ & {$[30+]$} & $\mathrm{F}$ & & & & & & & & - & & & & & & & & & & & & & & & & & & & & & & & & & & & & \\
\hline $127>$ & 1 & & {$[30+$} & $\mathrm{M}$ & A & & & - & NO & & $\overline{\mathrm{A}}$ & No & 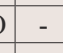 & $\mathrm{NO}$ & A & & A & & & B & NO & & A & - & - & & & \begin{tabular}{|l|}
- \\
\end{tabular} & & NO & & NO & & & & $\mathrm{NO}$ & & as de lésion & Pas de lésion & as de lésio \\
\hline 12 & $\mathrm{AD}$ & ans & Fit & & & & & & & & & & & & & & & & & & & & & & & & & & & & & & & & & & & & & \\
\hline
\end{tabular}
AD Adulte IM
M S
$\mathrm{F}$ Sexe féminin
- Él
$\mathrm{NO}$

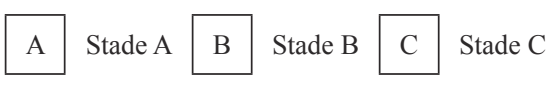


Annexe 2B. Données paléobiologiques individuelles pour l'échantillon de la Cathédrale (3/5)

\begin{tabular}{|c|c|c|c|c|c|c|c|c|c|c|c|c|c|c|c|c|c|c|c|c|c|c|c|c|c|c|c|c|c|c|c|c|c|c|c|c|c|c|c|}
\hline Sandetto $>$ & & Age & & & & & oplas & sie m & aaxil & laire & droit & & & Нуро & plasi & sie ma & axillai & ire ga & auche & & & Нур & oplas & ie me & andit & bule d & droite & & & Нуро & plasi & ie ma & andib & bule g & gauch & & Jasie & & Cribra orbitalia \\
\hline squeterte & Categorie & Estimation & Classe & & M3 & M2 & M1 & P2 & P1 & C & 12 & I1 & I1 & 12 & C & P1 & P2 & M1 & M2 & M3 & M3 & \begin{tabular}{|l|l|}
3 M22 \\
\end{tabular} & M1 & P2 & P1 & C & 12 & I1 & I1 & 12 & C & P1 & P2 & M1 & 1 M2 & \begin{tabular}{|l|l|}
2 & M3 \\
\end{tabular} & individu & Orbite droite & Orbite gauche \\
\hline 130 & IM & $4-6$ ans & {$[1-4]$} & I & & - & & & - & - & & & & - & - & - & - & & & & - & - & & - & & - & & - & & - & - & - & - & & & & & & \\
\hline 131 & $\mathrm{AD}$ & $>20$ ans & {$[20+]$} & I & & & & & - & & & 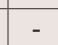 & & & & - & - & 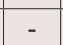 & & & 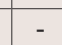 & - & & - & - & - & - & - & - & - & 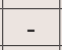 & $x^{-5}+2$ & - & - & - & & & & \\
\hline 133 & $\mathrm{AD}$ & $>50$ ans & {$[30+]$} & $\mathrm{M}$ & & - & - & - & - & - & & - & - & & A & A & B & & & - & - & - & & A & - & - & - & - & - & - & - & - & - & - & - & - & B & Pas de lésion & Pas de lésion \\
\hline 134 & D & 30-50 ans & {$[30+]$} & M & - & - & NO & NO & $\mathrm{NO}$ & $\mathrm{NC}$ & NO & NO & NO & $\mathrm{NO}$ & NO & $\mathrm{NO}$ & NO & - & 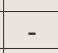 & - & $\mathrm{A}$ & B & NO & NO & $\mathrm{NO}$ & NO & NO & NO & NO & NO & B & B & NO & $\mathrm{NO}$ & $\mathrm{NO}$ & & B & \begin{tabular}{|l|} 
Pas de lésion \\
\end{tabular} & Pas de lésion \\
\hline 135 & M & $12-15$ ans & {$[10-14]$} & I & - & - & - & - & - & - & - & - & - & - & - & - & - & - & - & - & - & - & - & - & - & - & - & - & - & - & - & - & - & - & - & - & 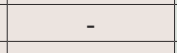 & & - \\
\hline 136 & $\mathrm{D}$ & $>20$ ans & {$[20+]$} & I & - & - & & - & - & - & & - & & - & & - & & & - & - & - & - & - & - & - & - & - & - & - & - & - & - & - & - & - & - & & & \\
\hline 137 & IM & 4-6 ans & {$[1-4]$} & I & - & & A & & - & - & - & A & B & - & - & - & - & B & & - & - & - & - & - & - & - & - & - & - & - & - & - & - & - & - & - & $\mathrm{B}$ & Présence & Présence \\
\hline 138 & D & $>40$ ans & {$[30+]$} & M & - & - & & - & - & - & - & & - & - & - & - & - & & - & - & - & - & - & - & - & - & - & - & - & - & - & - & - & - & - & - & & Pas de lésion & Pas de lésion \\
\hline 139 & $\mathrm{D}$ & $>20$ ans & {$[20+]$} & I & - & - & - & - & - & - & & - & - & - & - & - & - & & - & - & - & - & - & - & - & - & - & - & - & - & - & - & - & - & - & - & - & & \\
\hline 140 & $\mathrm{AD}$ & $>20$ ans & {$[20+]$} & $\mathrm{I}$ & - & - & - & - & - & - & - & - & - & - & - & - & - & - & - & - & - & - & - & - & - & - & - & - & - & - & - & - & - & - & - & - & - & & \\
\hline 141 & $\mathrm{AD}$ & $20-40$ ans & {$[20+]$} & M & - & $\mathrm{A}$ & B & NO & $\mathrm{NO}$ & - & - & - & A & A & NO & $\mathrm{NO}$ & NO & - & - & - & - & B & $y^{-\infty}+2+$ & A & A & B & B & - & - & - & B & B & B & NO & $\mathrm{NO}$ & $5-$ & B & \begin{tabular}{|l|} 
Pas de lésion \\
\end{tabular} & Pas de lésion \\
\hline 142 & IM & 11-14 ans & {$[10-14]$} & $I$ & & 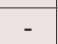 & -1 & $\mathrm{E}$ & 5 & & & - & & & & 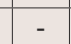 & 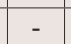 & & & & - & & & - & & & & - & - & - & - & & - & 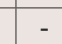 & & & & & \\
\hline 143 & $\mathrm{AD}$ & 2. & {$[20$} & $\mathrm{M}$ & - & - & 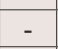 & - & - & - & - & - & - & - & - & - & - & - & - & - & - & - & - & - & - & - & - & - & - & - & - & - & - & - & - & - & & & 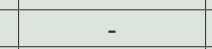 \\
\hline 144 & $\mathrm{AD}$ & 25 & {$[20+]$} & Fin & - & - & - & - & - & - & - & - & - & - & $x_{-1}+2 x$ & - & - & 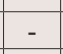 & - & - & $\mathrm{A}$ & A & A & $\mathrm{A}$ & $\mathrm{A}$ & A & A & NO & B & NO & A & $\mathrm{A}$ & $\mathrm{A}$ & A & A & A & $\mathrm{E}$ & & \\
\hline 145 & $\mathrm{AD}$ & $20-30$ ans & [20-29] & M & - & - & - & - & - & - & - & - & - & - & - & - & - & - & - & - & - & - & - & - & - & - & - & - & - & - & - & - & - & - & - & - & - & & \\
\hline 147 & IM & 9-12 mois & {$[0]$} & I & - & & & & & & - & & - & - & & & - & - & & & & - & 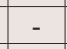 & & & & & & & & & & & & & & - & $\mathrm{s}$ de lésion & Présence \\
\hline $148 / 1$ & $\mathrm{AD}$ & $>40$ ans & {$[30+]$} & F? & & B & C & NO & B & B & & & A & - & A & $\mathrm{NO}$ & - & 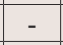 & B & A & $\mathrm{A}$ & A & & A & B & C & NO & $\mathrm{NO}$ & NO & $\mathrm{NO}$ & B & B & NO & $\mathrm{NO}$ & \begin{tabular}{|l|l|}
5 & $B$ \\
\end{tabular} & $\mathrm{NO}$ & $\mathrm{C}$ & as de lésion & Pas de lésion \\
\hline $148 / 2$ & IM & $2-4$ ans & {$[1-4]$} & I & - & - & - & - & - & - & - & - & - & - & - & - & - & - & - & - & - & - & - & - & - & - & - & - & - & - & - & - & - & - & - & - & - & Pas de lésion & \\
\hline 149 & IM & $2-4$ ans & {$[1-4]$} & I & - & - & - & - & - & - & - & - & - & - & - & - & - & - & - & - & - & - & - & - & - & - & - & - & - & - & - & - & - & - & - & 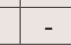 & & & \\
\hline 150 & $\mathrm{AD}$ & $30-50$ ans & {$[30+]$} & M & - & - & - & - & - & - & - & - & - & - & - & - & - & - & - & - & - & - & - & - & - & - & - & - & - & - & - & - & - & - & - & - & - & - & - \\
\hline 151 & $\mathrm{AD}$ & $30-60$ ans & {$[30+]$} & M & - & - & - & - & - & - & - & - & - & - & - & - & - & - & - & - & - & - & - & - & - & - & - & - & - & - & - & - & - & - & - & - & - & - & - \\
\hline 152 & IM & 18-24 mois & {$[1-4]$} & I & - & 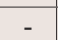 & - & - & - & - & - & - & - & - & - & - & $-\left.\right|_{-1}$ & $-1-$ & - & - & - & - & - & - & - & - & - & - & - & - & - & - & - & - & - & 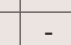 & - & résence & \\
\hline 153 & & 20 & {$[20-29]$} & I & - & - & 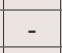 & - & - & - & & - & - & -5 & 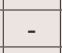 & - & - & - & & t & - & - & & - & - & - & - & - & - & - & 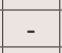 & - & - & - & 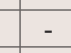 & - & - & & 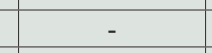 \\
\hline 15 & & & [0. & $=4$ & 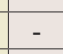 & - & - & - & - & - & - & - & - & - & 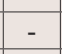 & - & - & - & - & & - & - & - & - & - & - & - & ${ }^{2}+2$ & - & - & - & 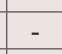 & - & - & - & $-5+2>$ & & ésen & résen \\
\hline 155 & $\mathrm{AD}$ & $20-30$ ans & {$[20-29]$} & M & - & - & - & - & - & - & - & - & - & - & - & - & - & - & - & - & - & - & - & - & - & - & - & - & - & - & - & - & - & - & - & - & - & & \\
\hline 156 & IM & 3-9 mois & {$[0]$} & I & - & - & G & - & - & - & - & - & - & - & - & - & - & - & - & - & - & - & - & - & - & - & 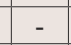 & - & - & - & - & - & - & - & - & & 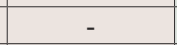 & & \\
\hline 158 & $\mathrm{AD}$ & $40-60$ ans & {$[30+]$} & M? & NO & NO & NO & NO & $\mathrm{NO}$ & $\mathrm{NC}$ & No & NO & $\mathrm{NO}$ & NO & NO & NO & No & NO & NO & NO & $\mathrm{A}$ & $\mathrm{A}$ & $\mathrm{A}$ & NO & NO & NO & NO & NO & NO & NO & B & NO & NO & $\mathrm{NO}$ & DO & DO & B & \begin{tabular}{|l|} 
Pas de lésion \\
\end{tabular} & Pas de lésion \\
\hline 159 & $\mathrm{AD}$ & $>20$ ans & {$[20+]$} & I & - & - & - & - & - & - & - & - & - & - & - & - & - & - & - & - & - & - & - & - & - & - & - & - & - & - & - & - & - & - & - & - & - & - & - \\
\hline 160 & $\mathrm{AD}$ & $20-30$ ans & {$[20-29]$} & M & - & - & - & - & - & - & - & - & - & - & - & - & - & - & - & - & - & - & - & - & - & - & - & - & - & - & - & - & - & - & - & & & & \\
\hline 161 & & $40-60$ ans & {$[30+]$} & $\mathrm{M}$ & - & - & NO & A & A & $\mathrm{NC}$ & NO & B & C & NO & NO & $\mathrm{NO}$ & A & - & - & - & NO & B & NO & A & A & A & A & B & B & A & A & A & A & NO & $\mathrm{NO}$ & $\mathrm{NO}$ & $c$ & \begin{tabular}{|l|} 
Pas de lésion \\
\end{tabular} & Pas de lésion \\
\hline 162 & & 38 & {$[30$} & $\mathrm{M}$ & - & - & - & & - & - & & & - & - & - & - & - & - & - & - & - & - & - & - & - & - & - & & 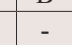 & - & - & - & - & 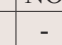 & - & & & & \\
\hline 163 & $\mathrm{IM}$ & & {$[15-19]$} & $\mathrm{F}$ & & B & $\mathrm{A}$ & $\mathrm{A}$ & B & - & & - & - & -1 & & B & B & A & NO & NO & $\mathrm{B}$ & $\mathrm{C}$ & NO & $\mathrm{A}$ & B & B & B & A & $\mathrm{NO}$ & B & $\mathrm{A}$ & $\mathrm{A}$ & A & $\mathrm{A}$ & $\mathrm{B}$ & NO & $c$ & is de lésion & Pas de lésion \\
\hline 16 & & & {$[20+]$} & & - & 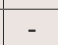 & - & - & - & - & - & - & - & -1 & . & - & - & - & es 5 & - & - & - & & - & - & - & & - & - & - & - & - & - & - & - & & & & \\
\hline 16 & & $24-30$ & {$[1-4$} & & & - & - & 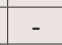 & - & 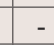 & & & - & - & & & - & & & & - & -5 & & - & 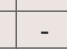 & & & - & - & & & - & - & - & & & & & \\
\hline 16 & $\mathrm{AL}$ & $>20$ ans & {$[20+$} & 1 & - & - & - & 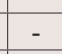 & - & - & - & - & - & - & - & - & - & - & - & - & - & - & - & - & - & - & - & - & - & - & - & - & - & - & - & & & & \\
\hline 16 & $\mathrm{AL}$ & $20-40$ ans & {$[20+$} & $\mathrm{N}$ & - & - & - & - & - & - & - & - & - & - & - & - & - & - & - & - & - & - & - & - & - & - & - & - & - & - & - & - & - & - & - & - & - & & - \\
\hline 16 & A & $>20$ ans & {$[20+$} & I & & & & & & - & & & - & - & & & - & - & & & - & - & & & - & - & & & - & - & - & - & - & - & - & 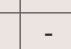 & - & & \\
\hline 17 & & & {$[36$} & $I$ & - & - & - & - & - & - & - & - & - & - & - & - & -1 & - & - & - & - & - & - & - & - & - & - & - & - & $1-$ & -1 & -1 & - & $1-$ & - & - & - & \begin{tabular}{|l|} 
Pas de lésion \\
\end{tabular} & Pas de lésion \\
\hline & & 31 & {$[36$} & I & & & - & & - & - & & - & - & - & & & - & - & & & - & - & & - & - & - & & & - & - & - & - & - & - & - & & & & \\
\hline & & 40 & {$[30$} & M & NO & NO & & NO & B & B & NO & & NO & $\mathrm{NO}$ & B & B & & NO & NO & NO & $\mathrm{NO}$ & NO & NO & NO & $\mathrm{NO}$ & B & NO & NO & & NO & B & B & A & NO & $\mathrm{NO}$ & $\mathrm{NO}$ & 1 & Pas de lésion & Pas de lésion \\
\hline 17 & & & {$[1$.} & I & - & - & - & - & - & - & & - & - & - & & & - & - & & - & - & - & & - & - & & & - & - & - & - & - & - & - & - & & & & \\
\hline 174 & & $20-40$ ans & {$[20+]$} & $\mathrm{M}$ & - & 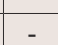 & - & - & - & 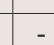 & - & - & - & - & - & - & - & - & - & - & - & - & - & - & - & 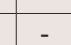 & & - & - & - & - & - & - & 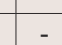 & - & - & - & & \\
\hline 17 & & $>$ & $\frac{[20+]}{[30+]}$ & $\frac{\mathrm{N}}{\mathrm{I}}$ & - & - & - & - & - & - & - & - & - & - & - & - & - & - & - & - & - & - & $-2+2>$ & - & - & - & - & - & - & - & - & - & - & - & - & - & & & \\
\hline 17 & & & {$[26$} & 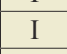 & I & - & - & - & - & - & - & - & - & - & & - & - & - & & - & - & - & - & 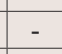 & - & - & +2 & 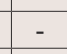 & - & - & - & 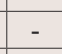 & - & - & 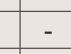 & & & $\mathrm{N}$ & $\mathrm{NC}$ \\
\hline 17 & & & {$[2$} & & & - & -2 & - & - & 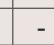 & - & - & - & - & & 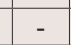 & - & 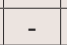 & & - & - & $\pi$ & & - & - & - & & 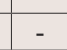 & 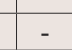 & 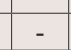 & & & - & & & & & & \\
\hline 178 & $\mathrm{~A}$ & & {$[20$} & M & $\mathrm{C}$ & NO & NO & NO & $\mathrm{NO}$ & $\mathrm{B}$ & NO & NO & $\mathrm{C}$ & B & NO & $\mathrm{NO}$ & NO & NO & NO & B & B & NO & A & A & B & NO & $\mathrm{NO}$ & NO & NO & B & B & B & $\mathrm{A}$ & NO & $5 \mathrm{NO}$ & j & & s de lésion & \\
\hline 17 & A & & {$[36$} & $\mathrm{N}$ & NO & Ne & & - & - & $\mathrm{NC}$ & A & NO & NO & $\mathrm{NO}$ & NO & & - & - & - & NO & $\mathrm{A}$ & NO & & & $\mathrm{NO}$ & NO & NO & NO & NO & NO & NO & NO & $\mathrm{NO}$ & ) & & NO & 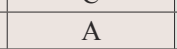 & de lesion & Pas de lésion \\
\hline 18 & A & & {$[3$} & $\mathrm{N}$ & I & E & - & 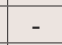 & 2 & 2 & & - & - & - & -2 & 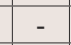 & - & 5 & E & $2-5$ & & & . & 2 & - & & & & -1 & & - & $\mathrm{E}$ & $\mathrm{A}$ & $\mathrm{A}$ & $\mathrm{A}$ & $\mathrm{A}$ & 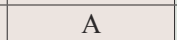 & & \\
\hline 18 & & & {$[30$} & M & & - & - & 5 & - & & & $2-$ & - & - & & & - & 5 & & - & $\mathrm{NO}$ & NO & & 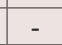 & A & $\mathrm{C}$ & B & NO & NO & NO & B & A & & & $\mathrm{NO}$ & $\mathrm{NO}$ & ( & s de lésion & as de lésion \\
\hline 182 & IM & ins & {$[15-19]$} & I & A & B & NO & NO & $\mathrm{NO}$ & $\mathrm{NC}$ & NO & A & $\mathrm{A}$ & NO & NO & NO & A & NO & B & A & $\mathrm{A}$ & B & B & A & A & NO & NO & NO & NO & NO & NO & NO & $\mathrm{A}$ & B & $\mathrm{A}$ & $\mathrm{A}$ & B & Pas de lésion & Pas de lésion \\
\hline 184 & IM & $0-6$ mois & {$[0]$} & I & & & $1+5$ & 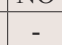 & - & - & & 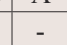 & - & - & 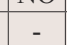 & - & - & 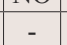 & 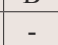 & 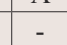 & $\pi$ & ${ }^{5}$ & & 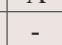 & - & - & 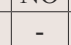 & 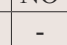 & - & - & - & $+\infty$ & - & 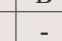 & - & $2 \pi$ & & & \\
\hline 18 & $\mathrm{AL}$ & $>40$ ans & {$[30+]$} & I & & - & 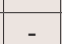 & 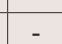 & & - & - & 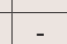 & & - & 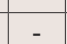 & - & - & - & & & & 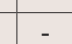 & - & 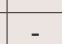 & 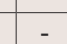 & 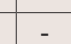 & 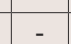 & 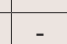 & - & - & 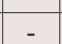 & 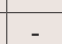 & & & 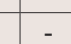 & & & & \\
\hline & & $40-60$ & {$[30-$} & M & B & B & NO & NO & NO & B & NO & NO & B & 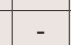 & B & NO & NO & B & A & B & A & B & 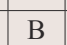 & A & A & NO & C & B & NO & $\mathrm{C}$ & A & B & A & B & 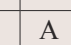 & & & & \\
\hline & & & {$[30-$} & -3 & NO & & No & A & $\mathrm{A}$ & $\mathrm{A}$ & A & B & B & $\mathrm{A}$ & $\Delta$ & Not & $\mathrm{A}$ & NO & A & NO & NO & $\mathrm{C}$ & & $\mathrm{A}$ & A & B & $\mathrm{A}$ & t & A & B & A & 0 & rit & D & $\mathrm{C}$ & B & & & de lésion \\
\hline & & & & $F^{2}$ & & & & & - & & & & & & & & $-5+2>$ & & & & - & & & & & & & & & & & & & & & & & & \\
\hline & & & & & & & & & & & & & & & & & & & & & & & & & & & & & & & & & & & & & & & \\
\hline 190 & $\mathrm{AD}$ & $30-60$ ans & {$[30+]$} & $\mathrm{M}$ & & & & $\mathrm{NO}$ & $\mathrm{NO}$ & $\mathrm{A}$ & NO & $\mathrm{NO}$ & $\mathrm{NO}$ & $\mathrm{NC}$ & & $\mathrm{NO}$ & $\mathrm{NC}$ & & & & $\mathrm{NO}$ & NO & & A & $\mathrm{A}$ & NO & $\mathrm{NC}$ & & NO & B & A & & NO & & $\mathrm{NO}$ & & D & Pas de lésion & Pas de lésion \\
\hline
\end{tabular}

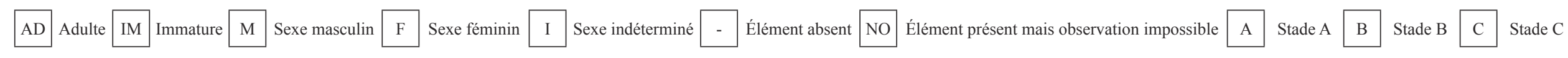


Annexe 2B. Données paléobiologiques individuelles pour l'échantillon de la Cathédrale (4/5)

\begin{tabular}{|c|c|c|c|c|c|c|c|c|c|c|c|c|c|c|c|c|c|c|c|c|c|c|c|c|c|c|c|c|c|c|c|c|c|c|c|c|c|c|c|}
\hline & & Age & & & & Нурор & plasii & ie max & xillair & re droit & & & Нypop & lasie & max & xillair & re gaus & & & Нуро & oplasi & ie mand & indibul & ule droite & & & Hypopla & lasie ma & andibu & ule gat & uuche & & ie & & Cribra orbitalia & & & Réaction périost & \\
\hline & ategorie & Estimation & Classe & & M3 & \begin{tabular}{l|l} 
M2 & M
\end{tabular} & \begin{tabular}{l|l} 
M1 & I
\end{tabular} & \begin{tabular}{l|l|} 
P2 & 1 \\
\end{tabular} & \begin{tabular}{l|ll} 
P1 & $C$ \\
\end{tabular} & \begin{tabular}{c|l} 
C & 12 \\
\end{tabular} & \begin{tabular}{|l|l|} 
II \\
\end{tabular} & I1 & 12 & C & P1 & \begin{tabular}{l|l}
$\mathbf{P 2}$ & $\mathrm{I}$ \\
\end{tabular} & \begin{tabular}{l|l} 
M1 & N \\
\end{tabular} & \begin{tabular}{l|l} 
M2 & M
\end{tabular} & $13 \mathrm{M3}$ & M2 & M1 & \begin{tabular}{|l|l|} 
P2 & P \\
\end{tabular} & \begin{tabular}{l|l} 
P1 & C \\
\end{tabular} & \begin{tabular}{l|l} 
C & I2
\end{tabular} & I1 & I1 & \begin{tabular}{|l|l|} 
I2 & C \\
\end{tabular} & \begin{tabular}{l|l} 
C & P1
\end{tabular} & 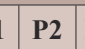 & M1 1 & \begin{tabular}{l|l} 
M2 1 \\
1
\end{tabular} & M3 & dividu & Orbite droite & Orbite gauche & Individu & Tibia droit & Tibia gauche & Tibia \\
\hline 191 & $\mathrm{AD}$ & $>20$ ans & {$[20+]$} & M? & & & & & & & & & & & & & & & & & & & & & & & & & & & & & & & & & Remodelée & Pas de lésion & Remodelée \\
\hline 192 & $\mathrm{AD}$ & $20-25$ ans & [20-29] & M? & - & - & - & - & - & - & - & & & & - & -5 & & 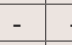 & $\mathrm{B}$ & C & $\mathrm{A}$ & \begin{tabular}{|l|l}
$\mathrm{A}$ & $\mathrm{B}$ \\
\end{tabular} & \begin{tabular}{l|l} 
B & I \\
\end{tabular} & \begin{tabular}{l|l} 
B & B \\
\end{tabular} & NO & NO & \begin{tabular}{|l|l} 
B & B \\
\end{tabular} & \begin{tabular}{l|l}
$B$ & A \\
\end{tabular} & $\mathrm{NO}$ & NO & B & B & $\mathrm{C}$ & & & & & & \\
\hline 193 & M & $16-$ & [15-19] & & & $-e_{-1}^{-}$ & - & - & & & - & & & & 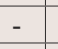 & & & 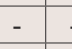 & $-{ }_{-}^{-}$ & - & - & $-e_{-1}^{-}$ & - & & t & & & - & & - & & & & & & & & & \\
\hline 194 & D & $20-$ & {$[20+]$} & I & & - & & - & - & & - & & & & - & - & & & - & - & - & - & & - & - & - & - & - & - & - & & - & - & & & & is de lésion & Pas de lésion & as de lésion \\
\hline 195 & M & $16-$ & {$[15-19]$} & I & - & $-e_{-1}^{-}$ & - & - & - & -5 & - & - & - & - & - & - & $-e^{-}$ & $-e_{-}^{-}$ & $-1-$ & - & - & \begin{tabular}{|c|c}
- & - \\
\end{tabular} & $-x_{-1}$ & \begin{tabular}{l|l}
- & - \\
- & -1
\end{tabular} & - & - & \begin{tabular}{|l|l|}
- & - \\
\end{tabular} & $\begin{array}{l}- \\
-\end{array}$ & - & - & - & - & - & - & - & - & $s$ de lésion & - & Pas de lésion \\
\hline 196 & $\mathrm{AD}$ & $20-5$ & {$[20+]$} & M & & $-e_{-1}^{-}$ & - & - & - & $\begin{array}{l}- \\
-\end{array}$ & - & - & - & - & - & - & $-e_{-1}$ & $-e^{-}$ & $-{ }_{-}^{-}-$ & - & - & $-e_{-1}^{-}-$ & - & & & - & \begin{tabular}{|l|l|}
- & - \\
\end{tabular} & $\begin{array}{l}- \\
-\end{array}$ & - & & - & - & & & & & & & \\
\hline 197 & IM & 8. & {$[5-9]$} & I & & & & & & $\begin{array}{l}- \\
-\end{array}$ & - & & & & & - & & & & - & B & & & B & B & B & - & $\begin{array}{l}- \\
-\end{array}$ & - & B & & - & B & as de lésion & Pas de lésion & as de lésion & & & \\
\hline 198 & $\mathrm{Al}$ & $40-60$ ans & {$[30+]$} & M & - & $-\mathrm{N}$ & \begin{tabular}{l|l}
$\mathrm{NO}$ & $\mathrm{N}$ \\
$\mathrm{n}$
\end{tabular} & NO 1 & vo & $--_{-}^{-}$ & - & - & - & No 1 & No & - & \begin{tabular}{l|l}
- & 1 \\
-
\end{tabular} & B & - NO & - & & \begin{tabular}{|l|l}
$\mathrm{A}$ & $\mathrm{C}$ \\
\end{tabular} & $\begin{array}{ll}\mathrm{C} \\
\mathrm{C}\end{array}$ & \begin{tabular}{l|l}
- & - \\
\end{tabular} & - & - & & $--_{-}^{-}$ & - & - & A & - & $\mathrm{C}$ & Pas de lésion & Pas de lésion & Pas de lésion & & - & \\
\hline 199 & $\mathrm{AD}$ & $30-50$ ans & {$[30+]$} & M & & - & & - & - & - - - & - & - & - & - & - & - & & 2 t & -1 & - & - & $-{ }_{-1}-$ & & $--_{-}^{-}$ & - & 3 & & $--_{-}^{-}$ & - & S & & - & & & & & Remodelée & & Remodelée \\
\hline 200 & $\mathrm{AD}$ & $20-30$ ans & [20-29] & $\mathrm{F}$ & B & \begin{tabular}{l|l}
$\mathrm{B}$ & $\mathrm{N}$ \\
\end{tabular} & No & $\mathrm{A}$ & A & \begin{tabular}{l|l}
$\mathrm{A}$ & $\mathrm{A}$ \\
\end{tabular} & $1-$ & A & A & A & $\mathrm{A} \mid \mathrm{I}$ & NO 1 & \begin{tabular}{l|l} 
NO & 1 \\
\end{tabular} & \begin{tabular}{l|l} 
B & I \\
\end{tabular} & \begin{tabular}{l|l}
$\mathrm{B}$ & $\mathrm{A}$ \\
\end{tabular} & A & A & \begin{tabular}{|l|l}
$\mathrm{A}$ & $\mathrm{B}$ \\
\end{tabular} & $\begin{array}{ll}\text { B } & \text { C }\end{array}$ & \begin{tabular}{l|l}
$\mathrm{C}$ & $\mathrm{NO}$ \\
\end{tabular} & NO & NO & \begin{tabular}{|l|l|} 
B & B \\
\end{tabular} & \begin{tabular}{l|l} 
B & B \\
\end{tabular} & A & A & A & A & $\mathrm{C}$ & & Pas de lésion & Pas de lésion & Remodelée & - & Remodelée \\
\hline 201 & $\mathrm{AD}$ & $30-50$ ans & {$[30+]$} & M & C & \begin{tabular}{l|l} 
B & F \\
\end{tabular} & B & $\mathrm{A}$ & A & \begin{tabular}{l|l} 
B & B \\
\end{tabular} & B & B & & & - & t & - & -1 & $-\mathrm{A}$ & B & A & \begin{tabular}{|l|l} 
B & $\mathrm{N}$ \\
\end{tabular} & NO 1 & \begin{tabular}{l|l} 
B & B \\
\end{tabular} & NO & NO & \begin{tabular}{|l|l|} 
B & B \\
\end{tabular} & \begin{tabular}{l|l|}
$B$ & $B$ \\
\end{tabular} & B & B & A & A & $C$ & Pas de lésion & Pas de lésion & Pas de lésion & Pas de lésion & Remodelée & Remodelée \\
\hline 202 & $\mathrm{AD}$ & $30-50$ ans & {$[30+$} & M & & & & 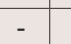 & & & - & & & & - & - & & & - & & & 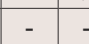 & & & 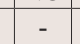 & & & & & & & & & & & & Remodelée & emodelée & Remodelée \\
\hline 203 & $\mathrm{AD}$ & $30-$ & {$[30$} & M & & - & & - & -5 & & - & - & & & - & - & & & NO & B & & \begin{tabular}{|l|l} 
B & E \\
\end{tabular} & \begin{tabular}{l|l}
$\mathrm{B}$ & $\mathrm{C}$ \\
\end{tabular} & $\mathrm{C}$ & - & $-t_{1}$ & NO NC & NO NO & \begin{tabular}{|l|l|} 
A \\
\end{tabular} & & B & NO & c & s de lésion & ss de lésion & $s$ de lésion & Remodelée & & emodelée \\
\hline 204 & $\mathrm{AI}$ & $>$ & {$[30$} & & & - & & - & - & & - & - & & & - & - & & - & - & & & $-x^{-}$ & & & - & & Nis & -- & & & & & & & & & & & \\
\hline 205 & $\mathrm{AD}$ & $>2$ & {$[20+]$} & I & - &.- & - & - & - & - - & - & - & - & - & - & - & $-{ }_{-}^{-}$ & $-{ }_{-}$ & $-{ }_{-}^{-}$ & - & - & -- & $-{ }_{-}-$ & - & - & - & $-{ }_{-1}^{-}-$ & $--_{-}^{-}$ & - & - & 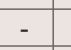 & - & - & & & - & - & nods & delée \\
\hline 206 & $\mathrm{AI}$ & $>2$ & {$[20+]$} & I & & - & & - & - & & & - & & & - & - & & $-{ }_{-}^{-}$. & - & 2 & - & $-{ }_{-1}^{-}$ & - & - & - & - & & $--_{-}^{-}$ & - & & 2 & & 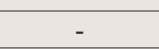 & & & & & & \\
\hline 207 & $\mathrm{AI}$ & $30-60$ ans & {$[30+]$} & M? & B & & \begin{tabular}{l|l}
$\mathrm{NO}$ & $\mathrm{N}$ \\
\end{tabular} & NO & & $\mathrm{NO} \mathrm{NO}$ & $\mathrm{ONO}$ & & $\mathrm{NO} / \mathrm{r}$ & No & & NO 1 & NO & & B & & 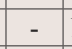 & \begin{tabular}{l|l} 
NO & $A$ \\
\end{tabular} & \begin{tabular}{l|l}
$\mathrm{A}$ & $\mathrm{B}$ \\
\end{tabular} & \begin{tabular}{l|l} 
B & B \\
\end{tabular} & NO & NO & \begin{tabular}{l|l} 
NO & $\mathrm{C}$ \\
\end{tabular} & \begin{tabular}{l|l}
$\mathrm{C}$ & $\mathrm{A}$ \\
\end{tabular} & $\mathrm{NO}$ & NO & A & B & $\mathrm{C}$ & $\mathrm{s}$ de lésion & de lésion & is de lésion & is de lésion & emodelée & Remodelée \\
\hline 209 & $\mathrm{AD}$ & $20-30$ ans & [20-29] & M & A & \begin{tabular}{l|l}
$\mathrm{A}$ & $\mathrm{N}$ \\
\end{tabular} & \begin{tabular}{l|l}
$\mathrm{NO}$ & $\mathrm{N}$ \\
$\mathrm{n}$
\end{tabular} & NO 1 & NO & \begin{tabular}{l|l}
$\mathrm{B}$ & $\mathrm{NO}$ \\
\end{tabular} & $\begin{array}{ll}\mathrm{O} & \mathrm{B} \\
\end{array}$ & B & $\mathrm{NO} / \mathrm{r}$ & No 1 & No & NO & - & - & $-\mathrm{A}$ & A & - & \begin{tabular}{|l|l} 
A & A \\
\end{tabular} & \begin{tabular}{l|l}
$\mathrm{A}$ & $\mathrm{B}$ \\
\end{tabular} & \begin{tabular}{l|l} 
B & B \\
\end{tabular} & NO & NO & \begin{tabular}{|l|l} 
B & C \\
\end{tabular} & \begin{tabular}{l|l}
$\mathrm{C}$ & $\mathrm{A}$ \\
\end{tabular} & $\mathrm{A}$ & NO & - & A & $C$ & s de lésion & Pas de lésion & Pas de lésion & & 2 & - \\
\hline 210 & $\mathrm{AD}$ & $20-30$ ans & [20-29] & $\mathrm{F}$ & & & & - & & & 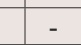 & & - & B & B & $\begin{array}{ll}A & \text { I }\end{array}$ & NO & A & & A & $\mathrm{A}$ & \begin{tabular}{|l|l} 
A & B \\
\end{tabular} & \begin{tabular}{l|l}
$\mathrm{B}$ & $\mathrm{B}$ \\
\end{tabular} & \begin{tabular}{l|l} 
B & B \\
\end{tabular} & B & NO & \begin{tabular}{l|l} 
C & C \\
\end{tabular} & $\begin{array}{ll}\mathrm{C} & \mathrm{C} \\
\end{array}$ & A & A & A & & $\mathrm{C}$ & $\mathrm{NO}$ & Pas de lésion & Pas de lésion & & & \\
\hline 212 & $\mathrm{AD}$ & $30-50$ ans & {$[30+]$} & M & - &.- & - & - & - & - - & - & - & - & - & - & - & - & - & -1. & - & - & -1 &.- & -- & - & - & -1. & $-1-$ & - & - & - & - & - & & & & Pas de lésion & - & Pas de lésion \\
\hline 213 & IM & 9-12 mois & {$[0]$} & $\mathrm{I}$ & - & - & - & - & - & - & - & - & - & - & - & - & - & - & - & 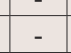 & - &.- & - & -1 & - & - & $-\quad-$ & -1 & - & - & - & - & - & - & & - & & - & Tas ac icsiont \\
\hline 214 & $\mathrm{AD}$ & $20-$ & {$[20-29]$} & $\mathrm{F}$ & & & & - & - & $-F_{-1}$ & - & -5 & - & - & - & - & - & - & - & - & - & -1 & -1 & - & - & - & -1 & -1 & - & - & 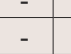 & - & - & & & & - & & \\
\hline 215 & & & {$[10$} & I & 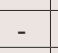 & & & - & - & - & - & - & & & - & - & & & $-{ }_{-}^{-}$ & - & & $-{ }_{-1}^{-}$ & - & & - & - & & $--_{-}^{-}$ & - & & - & - & & & & & & Mix & Mixte \\
\hline 216 & $\mathrm{AD}$ & & {$[30$} & $\mathrm{M}$ & & & \begin{tabular}{l|l}
$\mathrm{B}$ & $\mathrm{I}$ \\
\end{tabular} & NO & 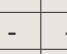 & & - & - & & NO & NO & NO & \begin{tabular}{l|l}
$\mathrm{C}$ & $\mathrm{I}$ \\
\end{tabular} & B & NO & & - & $\mathrm{NO} N$ & $\mathrm{NO} N$ & NO NO & NO & NO & $\mathrm{NO}$ NC & NO NO & $5-$ & - & - & - & $\mathrm{C}$ & s de lésion & $\mathrm{s}$ de lésion & as de lési & & & \\
\hline 217 & IN & & {$[10-1$} & $\mathrm{N}$ & - & 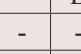 & - & - & - & - & - & - & - & - & - & - & - & - & - & - & - & $-\omega_{-1}^{-}$ & - & - & - & - & - & -- & - & - & - & - & - & - & Da r wirit & 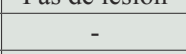 & - & de lésion & Pas de lésion \\
\hline 219 & $\mathrm{AD}$ & & [20-29] & M? & - & \begin{tabular}{l|l} 
B & $A$ \\
\end{tabular} & A & A & A & $\mathrm{NO}$ NO & $\mathrm{ONO}$ & NO & & No 1 & NO & A & \begin{tabular}{l|l}
$\mathrm{A}$ & $\mathrm{N}$ \\
\end{tabular} & \begin{tabular}{l|l}
$\mathrm{NO}$ & $\mathrm{N}$ \\
\end{tabular} & $\mathrm{O} / \mathrm{NO}$ & NO & A & \begin{tabular}{|l|l} 
A & A \\
\end{tabular} & \begin{tabular}{l|l}
$\mathrm{A}$ & $\mathrm{B}$ \\
\end{tabular} & \begin{tabular}{l|l}
$\mathrm{B}$ & $\mathrm{NO}$ \\
\end{tabular} & NO & NO & $\mathrm{NO}$ NC & \begin{tabular}{ll|l} 
SO & $B$ \\
\end{tabular} & A & $\mathrm{NO} / \mathrm{T}$ & NO & - & B & Pas de lésion & Pas de lésion & Pas de lésion & & & \\
\hline 220 & $\mathrm{AD}$ & $25-40$ ans & {$[20+]$} & M & & & & - & - & & 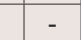 & & & & & & & & & & & & & & $2 t$ & & $E$ & & - & - & & - & & & & & \begin{tabular}{|l|} 
Pas de lésion \\
\end{tabular} & & Pas de lésion \\
\hline 221 & $\mathrm{~A}$ & 0 ans & {$[30+]$} & M & - & $-e_{-1}$ & - & - & - & $-{ }_{-1}^{-}$ & - & - & - & - & - & - & $-e_{-1}$ & - & $-{ }_{-1}^{-}$ & NO & NO & \begin{tabular}{l|l}
$\mathrm{NO}$ & $\mathrm{A}$ \\
\end{tabular} & \begin{tabular}{l|l}
$\mathrm{A}$ & $\mathrm{B}$ \\
\end{tabular} & \begin{tabular}{l|l}
$\mathrm{B}$ & $\mathrm{NO}$ \\
\end{tabular} & - & NO & NO NG & NO NO & $5-$ & $\mathrm{NO} / \mathrm{T}$ & NO & - & B & & & & - & - & - \\
\hline 222 & $\mathrm{AI}$ & ans & [20-29] & M & A & & $\mathrm{NO}$ & $\mathrm{A}$ & A & \begin{tabular}{l|l} 
B & A
\end{tabular} & $\mathrm{C}$ & $\mathrm{C}$ & $\mathrm{A}$ & $\mathrm{A}$ & & - & \begin{tabular}{l|l}
$\mathrm{A}$ & $\mathrm{B}$ \\
\end{tabular} & \begin{tabular}{l|l} 
B & $\mathrm{I}$ \\
\end{tabular} & \begin{tabular}{l|l}
$B$ & NO \\
\end{tabular} & A & NO & \begin{tabular}{|l|l} 
A & A \\
\end{tabular} & \begin{tabular}{l|l}
$\mathrm{A}$ & $\mathrm{A}$ \\
\end{tabular} & \begin{tabular}{l|l}
$\mathrm{A}$ & $\mathrm{C}$ \\
\end{tabular} & B & B & \begin{tabular}{|l|l} 
B & C \\
\end{tabular} & $\begin{array}{ll}\mathrm{C} & \mathrm{A} \\
\end{array}$ & $\mathrm{A}$ & $\mathrm{NO} / \mathrm{r}$ & NO & NO & c & as de lésion & Pas de lésion & as de lésion & & & \\
\hline 230 & & 20 & {$[20-29]$} & & & & & - & & & - & & & & B & A & NO N & NO & NO & NO & NO & -1 &.- & -- & - & 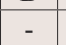 & $-1-$ & $-1-$ & - & - & - & - & B & as de lésion & Pas de lésion & Pas de lésion & NO & NO & \\
\hline 231 & & $>2$ & {$[20+]$} & I & - & - & - & - & - & - & - & - & - & - & - & - & - & - & - & 10 & & -1 & - & -1 & - & - & -1 & -- & - & - & - & & $D$ & & I as ue testont & & Pas de lésion & emodelée & Remodelée \\
\hline 232 & & & {$[30+]$} & I & A & \begin{tabular}{l|l} 
NO & $E$ \\
\end{tabular} & B & A & A & & - & & & B & $\mathrm{NO} /$ & NO 1 & NO & & B & & & NO $\mathrm{N}$ & \begin{tabular}{l|l} 
NO & $A$ \\
\end{tabular} & \begin{tabular}{l|l}
$A$ & $B$ \\
\end{tabular} & NO & NO & \begin{tabular}{l|l} 
C & B \\
\end{tabular} & $\begin{array}{ll}\mathrm{B} & \mathrm{NO} \\
\end{array}$ & NO & & NO 1 & NO & & de lésion & se lésion & lésion & & & \\
\hline 233 & & & {$[20-$} & & 2 & $x^{2}+2$ & & - & - & & - & - & 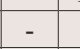 & & - & - & $x^{2}+2>$ & - & $-1-$ & & - & -1. &.- & & - & - & -1 & -1 & - & $t$ & & & & & & & lésion & & as de lésion \\
\hline 234 & $\mathrm{AD}$ & & {$[30+$} & & & & B & NO 1 & NO & NO NO & $\mathrm{O}$ NO & & & NO & & NO & & & & NO & & & & NO NO & NO & NO & $\mathrm{NO}$ NC & NO NO & $5 \mathrm{NO}$ & & NO & & & & & & & & \\
\hline 235 & $\mathrm{Al}$ & 40 & {$[30+]$} & $\mathrm{M}$ & - & - & - & - & - & $-\quad-$ & - & - & - & - & - & - & - & - & - & - & - & $-c_{-1}^{-}$ & - & - & - & - & - & - & - & - & - & - & & $s$ de lésion & de lésion & 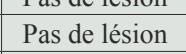 & NO & & \\
\hline 236 & $\mathrm{AI}$ & & {$[20+]$} & I & - & & & & & & & & & & & & & & & & & & & & & & & & & - & & & & & & & Pas de lésion & Pas de lésion & Pas de lésion \\
\hline 237 & $\mathrm{AI}$ & 30 & {$[30+]$} & M? & & & & A & A & \begin{tabular}{l|l}
$\mathrm{N} O$ \\
\end{tabular} & $\mathrm{O}$ NO & NO & NO & B & & A & & NO & B & A & & \begin{tabular}{l|l} 
NO & $A$ \\
\end{tabular} & \begin{tabular}{l|l}
$A$ & $N$ \\
\end{tabular} & NO NO & NO & NO & $\mathrm{NO}$ NC & so $\mathrm{B}$ & $\mathrm{A}$ & & A & & B & Pas de lésion & Pas de lésion & Pas de lésion & 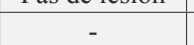 & & \\
\hline 238 & & & {$[20+]$} & & - & & & - & - & & - & - & & & - & & & & $-{ }_{-1}^{-}$ & & & $-{ }_{-1}^{-}$ & & \begin{tabular}{l|l}
- & - \\
\end{tabular} & - & - & \begin{tabular}{|l|l|}
- & - \\
\end{tabular} & 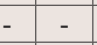 & - & - & & - & & & & & - & - & - \\
\hline 239 & & $40-$ & {$[30$} & $\mathrm{M}$ & & \begin{tabular}{l|l} 
B & C
\end{tabular} & $\mathrm{C}$ & - & $\mathrm{C}$ & B - & NO & & B & & $\mathrm{NO} / \mathrm{I}$ & NO $\mathrm{I}$ & NO N & NO & - & B & B & \begin{tabular}{|l|l} 
B & E \\
\end{tabular} & B & 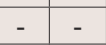 & - & - & $\begin{array}{ll}\text { C } & - \\
\end{array}$ & $-\mathrm{NO}$ & $\mathrm{NO}$ & $\mathrm{NO} / \mathrm{r}$ & NO & & $\mathrm{C}$ & as de lésion & Pas de lésion & Pas de lésion & - & & \\
\hline 243 & & & {$[20$} & & & & & - & & & - & - & & & - & & & & -1. & & & & & - & - & & & $-{ }_{-}^{-}$ & - & - & & - & & & & & & Pas de lésion & Pas de lésion \\
\hline 246 & & $20-$ & {$[20-29]$} & I & - & 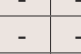 & - & - & - & $-1-$ & - & - & - & - & - & - & - & - & - & - & - & -1 & - & -1 & - & - & $-\quad-$ & -1 & - & - & - & - & - & - & - & - & Remodelée & s & Remodelée \\
\hline 247 & $\mathrm{AI}$ & $>2$ & {$[20+]$} & 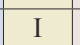 & & 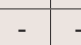 & & - & - & - & - & - & - & - & - & - &.- & - & -1. & - & - & \begin{tabular}{|l|l}
- & - \\
\end{tabular} &.- & $-{ }_{-}^{-}-$ & - & - & \begin{tabular}{l|l}
- & - \\
\end{tabular} & - - $_{-}$ & - & - & - & - & & & & - & - & & - \\
\hline 24 & $\mathrm{AI}$ & & {$[30$} & I & - & 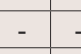 & - & - & - & - & - & - & & - & - & - & 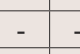 & $-{ }_{-}$ & $-{ }_{-}^{-}$ & & - & -- & -- & 2 & - & - & $-{ }_{-1}^{-}$ & $--_{-}^{-}$ & - & & - & - & & & & & & & - \\
\hline 25 & $\mathrm{AI}$ & & {$[30$} & $\mathrm{N}$ & & & & - & & & - & & & & & & & & & & & & & & & & & & & & & & & & & & & & \\
\hline 25 & & & {$[30$} & 10 & & $\mathrm{NO} N$ & NO 1 & NO 1 & NO & $\mathrm{NO}$ NO & $\mathrm{O}$ NO & - & & NO & NO $\mathrm{r}$ & $\mathrm{NO} \mathrm{I}$ & \begin{tabular}{l|l} 
NO & $A$ \\
\end{tabular} & A & A & NO & NO & \begin{tabular}{|l|l} 
B & B \\
\end{tabular} & B $\mathrm{NC}$ & NO NO & NO & NO & $\mathrm{NO}$ NC & SO B & \begin{tabular}{l|l}
$A$ & P
\end{tabular} & $\left.\mathrm{NO}\right|_{1}$ & NO & A & B & de lésion & Sde lé & de lés & & & \\
\hline 260 & $\mathrm{~A}$ & & {$[30$} & 1 & - & 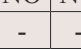 & - & - & $x+2>$ & & 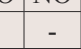 & - & & & - & - & & & - & & & 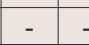 & -1. & (e) $>$ & - & 10 & & - & \begin{tabular}{l|l}
$A$ & $\mathrm{I}$ \\
\end{tabular} & NO & A & & A & de lésion & Pas de lésion & Pas de lésic & - & & \\
\hline 261 & $\mathrm{Al}$ & & {$[30$} & 1 & & & & & 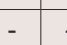 & & & & & & & & & & & & & & & & & & & & & & & & & & & & - & & \\
\hline 263 & & & {$[20$} & I & 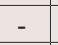 & $-e_{-1}$ & - & - & - & - & - & - & - & & - & - & - & - & - & - & & $-x_{-1}^{-}$ & -- & - & - & - & \begin{tabular}{|l|l|}
- & -
\end{tabular} & $--_{-1}$ & - & & & - & 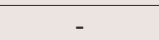 & & & - & - & & - \\
\hline 264 & & & {$[20+$} & I & &.- & - & - & - & & - & - & - & & - & - & 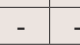 & 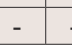 & - & - & & $-{ }_{-1}-$ & -- & & 20 & & \begin{tabular}{l|l}
- & - \\
\end{tabular} & 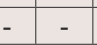 & - & & & & & & & - & & & \\
\hline 267 & $\mathrm{~A}$ & $30-60$ & {$[30+$} & & & - & - & - & - & & - & - & & & - & - & - & &.- & 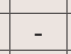 & & $--_{-1}^{-}$ &.- & $-1-$ & - & - & $-{ }_{-1}^{-}$ & $--_{-}^{-}$ & - & & & & & - & & - & Pas de lésion & Pas de lésion & \begin{tabular}{|l|l|} 
Pas de lésion \\
\end{tabular} \\
\hline 268 & $\mathrm{~A}$ & $>2$ & {$[20+$} & I & & - & - & - & - & $-1-$ & - & - & - & - & - & - & - & - & - & - & - & $--_{-1}$ & - & -1 & - & - & $-\quad-$ & -1 & - & & - & - & & - & - & - & - & as o c titit & \\
\hline 260 & $\mathrm{AI}$ & $20-40$ & {$[20+$} & 1 & & -1 & - & - & - & -1 & - & - & - & - & - & - & -1 & - & - & - & - & -1 & - & - & - & - & -1 & -1 & - & - & - & - & & & & & Pas de lésion & & Pas de lésion \\
\hline 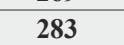 & & & L & & & - & - & -5 & -5 & & - & -5 & & & - & 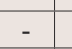 & & & -5 & & & $-7>$ & - & & 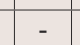 & - & & 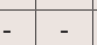 & - & & & & & & & & & & \\
\hline 28 & & & & & & & & & & & & & & & & & & & & & & & & & & & & & & & & & & & & & & & \\
\hline 28 & $\mathrm{~A}$ & & {$[20-$} & M? & A & A & B & B & A & \begin{tabular}{l|l}
$\mathrm{B}$ & $\mathrm{NO}$ \\
\end{tabular} & $\begin{array}{ll} \\
\end{array}$ & $\mathrm{C}$ & NO & B & NO & A & B & NO & \begin{tabular}{|l|l|}
$A$ & $B$ \\
\end{tabular} & B & B & \begin{tabular}{|l|l} 
B & $A$ \\
\end{tabular} & $\begin{array}{ll}\mathrm{A} & \mathrm{C} \\
\mathrm{C}\end{array}$ & $\begin{array}{ll}\mathrm{C} & \mathrm{NO}\end{array}$ & $\mathrm{NO}$ & NO & \begin{tabular}{|l|l} 
NO & B \\
\end{tabular} & \begin{tabular}{|l|l|} 
B & B \\
\end{tabular} & B & & & & $\mathrm{c}$ & de lésion & de lésion & $S$ de lésic & & & \\
\hline 286 & $\mathrm{AI}$ & & {$[36$} & I & 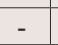 & & & - & & & 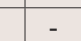 & & & & - & & & & -5 & & & $-{ }_{-1}-$ & - & & - & & & - & - & & & & & & & & & & \\
\hline 287 & $\mathrm{AD}$ & $40-60$ ans & {$[30+]$} & M? & & & & $\mathrm{NO} / \mathrm{N}$ & $\mathrm{NO} N$ & $\mathrm{NO}$ & $\mathrm{O}$ NO & NO & $\mathrm{NO} / \mathrm{N}$ & NO & $\mathrm{NO} / \mathrm{I}$ & $\mathrm{NO} / \mathrm{r}$ & $\mathrm{NO} N$ & & & NO & & \begin{tabular}{l|l}
$\mathrm{NO}$ & - \\
\end{tabular} & & \begin{tabular}{l|l}
$\mathrm{C}$ & $\mathrm{NO}$ \\
\end{tabular} & NO & $\mathrm{NO}$ & \begin{tabular}{l|l} 
NO & $\mathrm{C}$ \\
\end{tabular} & \begin{tabular}{l|l|}
$\mathrm{C}$ & $\mathrm{NO}$ \\
\end{tabular} & & & & & C & Pas de lésion & Pas de lésion & Pas de lésion & Remodelée & Remodelée & Remodelée \\
\hline
\end{tabular}

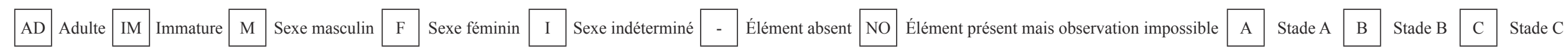


Annexe 2B. Données paléobiologiques individuelles pour l'échantillon de la Cathédrale (5/5)

\begin{tabular}{|c|c|c|c|c|c|c|c|c|c|c|c|c|c|c|c|c|c|c|c|c|c|c|c|c|c|c|c|c|c|c|c|c|c|c|c|c|c|c|c|}
\hline & & Age & & & & Нypo & plas & ie ma & axill: & aire d & & & & Нурор & blasie & e maxill & illaire g & gauche & & & Нурор & plasie n & mandib & bule d & droite & & & Hypoplas & lasie ma & andibu & ule gat & luche & & & & Cribra orbitalia & & & Réaction périosté \\
\hline & Categorie & Estimation & Classe & & M3 & M2 1 & M1 & P2 & P1 & C & \begin{tabular}{|l|}
12 \\
\end{tabular} & II & I1 & 12 & C & \begin{tabular}{l|l}
$\mathbf{P 1}$ & $\mathbf{P 2}$ \\
\end{tabular} & P2 2 M1 & 1 M2 & M3 & M3 & $\begin{array}{ll}\text { M2 } & \text { N }\end{array}$ & \begin{tabular}{l|l} 
M1 & P2
\end{tabular} & \begin{tabular}{l|l|}
2 & P1
\end{tabular} & C & 12 & I1 & \begin{tabular}{|l|l|} 
I1 & I \\
\end{tabular} & \begin{tabular}{l|l} 
I2 & C
\end{tabular} & \begin{tabular}{l|l} 
C & P1
\end{tabular} & \begin{tabular}{|l|l|} 
& P2 \\
\end{tabular} & M1 1 & $\begin{array}{lll}\text { M2 } & \text { I }\end{array}$ & M3 & individu & Orbite droite & Orbite gauche & Individu & Tibia droit & Tibia gauche \\
\hline 288 & $\mathrm{AD}$ & $30-50$ ans & {$[30+]$} & $\mathrm{M}$ & & - & - & - & - & & - & - & - & & & -5 & - & - & - & & $\mathrm{A} / \mathrm{I}$ & \begin{tabular}{l|l}
$\mathrm{A}$ & $\mathrm{A}$ \\
\end{tabular} & $\begin{array}{ll}\mathrm{A} & \mathrm{A} \\
\end{array}$ & B & A 1 & NO & & B & B - & -1 & A & 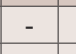 & & B & Pas de lésion & Pas de lésion & as de lésion & & \\
\hline 289 & $\mathrm{AD}$ & $30-$ & {$[30+]$} & I & -1 & - & & - & 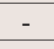 & & - & - & & - & & \begin{tabular}{l|l}
- & - \\
\end{tabular} & - & - & - & 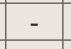 & - & -- & $\begin{array}{l}- \\
-\end{array}$ & 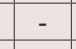 & 2 & - & - & 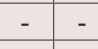 & $\begin{array}{l}- \\
-\end{array}$ & - & 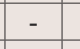 & - & - & - & 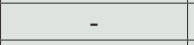 & & & Remodelée & Remodelée \\
\hline 291 & M & $16-$ & {$[15-19]$} & I & - & - & - & - & - & - & - & - & - & - & - & $-\quad-$ & - & - & - & - & - & $--_{-}^{-}$ & \begin{tabular}{l|l}
- & - \\
\end{tabular} & - & - & - & - & \begin{tabular}{l|l}
- & - \\
-
\end{tabular} & $--_{-}^{-}$ & - & - & -5 & - & - & - & - & - & Pas de lésion & Pas de lésion \\
\hline 293 & D & 30 & {$[30+]$} & $\mathrm{M}$ & & - & - & - & - & - & - & - & - & - & - & \begin{tabular}{l|l}
- & - \\
\end{tabular} & - - - & - & - & - & - & - - - & $-\quad-$ & - & - & - & - & & $-\quad-$ & - & & - & - & - & - & & - & & \\
\hline 294 & $\mathrm{AD}$ & 30 & {$[30+]$} & $\mathrm{M}$ & & & & - & - & - & - & - & - & - & - & \begin{tabular}{l|l}
- & - \\
\end{tabular} & - & - & - & - & - & $--_{-}^{-}$ & - & - & - & - & \begin{tabular}{l|l}
- & \\
\end{tabular} & \begin{tabular}{l|l}
- & - \\
\end{tabular} & \begin{tabular}{l|l}
- & - \\
\end{tabular} & - & - & & & - & & & & & \\
\hline 295 & $\mathrm{AD}$ & $30-50$ ans & {$[30+]$} & $\mathrm{M}$ & - & - & - & - & - & - & - & - & - & - & - & -- & -1 & - & - & - & - & -- & -- & - & - & - & -1 & -- & $--_{-}^{-}$ & - & - & - & - & - & - & - & & - & - \\
\hline 296 & IM & $16-18$ ans & {$[15-19]$} & $\mathrm{F}$ & A & $\mathrm{A} / \mathrm{P}$ & No & A & A & NO & A & - & A & B & NO $\mathrm{n}$ & $\begin{array}{lll}\text { NO } & \text { A } \\
\end{array}$ & $\begin{array}{l}A \\
\text { A NO }\end{array}$ & B & A & NO & $\begin{array}{ll}\mathrm{A} & \mathrm{F}\end{array}$ & $\begin{array}{ll}\mathrm{A} & \mathrm{B} \\
\end{array}$ & $\begin{array}{l}\text { B } \\
\text { B }\end{array}$ & B & NO 1 & NO & NO N & \begin{tabular}{l|l} 
NO & $B$ \\
\end{tabular} & \begin{tabular}{l|l}
$\mathrm{B}$ & $\mathrm{A}$ \\
\end{tabular} & \begin{tabular}{|l|} 
\\
\end{tabular} & A & A & B & B & Pas de lésion & Pas de lésion & Pas de lésion & & \\
\hline 297 & IM & Périnatal & [0] & $\mathrm{I}$ & - & - & -1 & -1 & - & $-a_{1}$ & - & -1 & -1 & - & - & $-{ }_{-}^{-}-$ & $-1-$ & - & $-\left.\right|_{1}$ & -1 & $-T_{1}$ & $-1-$ & $--_{-}^{-}$ & $-t_{1}$ & -1 & -1 & $-x_{1}$ & $--_{-}$ & $-1-$ & -1 & - & - & -1 & $E_{1}$ & & & & & \\
\hline 298 & $\mathrm{AD}$ & $30-50$ ans & {$[30+]$} & I & - & - & - & - & - & - & - & - & - & - & - & \begin{tabular}{l|l}
- & - \\
\end{tabular} & $\begin{array}{l}- \\
-\end{array}$ & - & - & - & - & -- & \begin{tabular}{l|l}
- & - \\
\end{tabular} & - & - & - & $-{ }^{-}-1$ & \begin{tabular}{l|l}
- & - \\
-
\end{tabular} & \begin{tabular}{l|l}
- & - \\
- & -
\end{tabular} & -1 & - & - & - & - & Pas de lésion & Pas de lésion & Pas de lésion & Pas de lésion & Pas de lésion \\
\hline 299 & $\mathrm{AD}$ & $20-40$ ans & {$[20+$} & 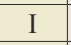 & & & & & & & & 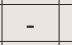 & & & & & & & $x_{1}$ & & & & & & & & & & & & & & & & & & & & \\
\hline 300 & A & & {$[30+$} & 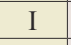 & - & - & - & - & - & - & 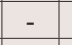 & - & - & - & - & \begin{tabular}{l|l}
- & - \\
\end{tabular} & - & 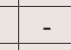 & - & & & - & $\begin{array}{l}- \\
-\end{array}$ & - & -5 & - & - & 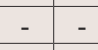 & $\begin{array}{l}- \\
-\end{array}$ & - & & & - & & & & - & emodelée & Remodelée \\
\hline 302 & $\mathrm{AD}$ & $>4$ & {$[30+]$} & I & & - & - & - & - & 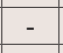 & - & - & - & - & - & \begin{tabular}{l|l}
- & - \\
\end{tabular} & $-{ }_{-}^{-}$ & -3 & - & A & B & -- & \begin{tabular}{l|l}
- & - \\
\end{tabular} & - & - & - & - & - & $\begin{array}{l}- \\
-\end{array}$ & $\mathrm{A}$ & $\mathrm{NO} /$ & NO & - & B & - & - & - & - & \\
\hline 303 & $\mathrm{AD}$ & $30-$ & {$[30+]$} & $\mathrm{M}$ & - & - & - & - & - & \begin{tabular}{|l|}
- \\
\end{tabular} & - & - & - & & - & \begin{tabular}{l|l}
- & - \\
\end{tabular} & $\begin{array}{l}- \\
-\end{array}$ & - & - & - & & & \begin{tabular}{l|l}
- & - \\
\end{tabular} & - & - & - & \begin{tabular}{l|l}
- & \\
\end{tabular} & \begin{tabular}{l|l}
- & - \\
\end{tabular} & \begin{tabular}{l|l}
- & - \\
\end{tabular} & - & & & - & - & - & - & - & - & - \\
\hline 304 & $\mathrm{AD}$ & $30-60$ ans & {$[30+]$} & $\mathrm{F}$ & - & - & - & - & - & - & - & - & - & - & - & - - & - & - & - & - & - & - - - & $--_{-}^{-}$ & - & - & - & $-e^{-}$ & $-\quad-$ & $-\quad-$ & - & - & - & - & - & - & - & - & - & \\
\hline 305 & $\mathrm{AD}$ & $20-40$ ans & {$[20+]$} & $\mathrm{F}$ & & & & - & - & & & & - & & & \begin{tabular}{l|l}
- & - \\
\end{tabular} & & & & & & & 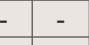 & & - & - & $-e^{-}$ & - & - & - & - & - & & - & - & & - & & \\
\hline 306 & $\mathrm{AI}$ & $25-40$ ans & {$[20+]$} & $\mathrm{F}$ & - & - & - & - & - & - & - & - & - & - & - & \begin{tabular}{l|l}
- & - \\
\end{tabular} & $-1-$ & - & - & - & - & -- & \begin{tabular}{l|l}
- & - \\
\end{tabular} & - & - & - & \begin{tabular}{l|l}
- & \\
\end{tabular} & \begin{tabular}{l|l}
- & - \\
-
\end{tabular} & \begin{tabular}{l|l}
- & - \\
\end{tabular} & - & - & - & - & - & - & - & - & Pas de lésion & Pas de lésion \\
\hline 307 & IM & 3-6 mois & {$[0]$} & I & - & - & 2 & - & - & - & - & $-{ }_{-1}$ & - & - & - & \begin{tabular}{l|l}
- & - \\
\end{tabular} & $\begin{array}{l}- \\
-\end{array}$ & - & - & - & - & -- & \begin{tabular}{l|l}
- & - \\
\end{tabular} & - & - & - & $-{ }^{-}$ & $--_{-}^{-}$ & \begin{tabular}{l|l}
- & - \\
\end{tabular} & - & - & - & - & & NO & NO & & & \\
\hline 308 & IM & 3-6 mois & [0] & I & - & - & - & - & - & - & - & - & - & - & - & \begin{tabular}{l|l}
- & - \\
\end{tabular} & $\begin{array}{l}- \\
-\end{array}$ & - & - & - & - & $--_{-}^{-}$ & $\begin{array}{l}- \\
-\end{array}$ & - & - & - & $-{ }^{-}$ & $--_{-}^{-}$ & \begin{tabular}{l|l}
- & - \\
\end{tabular} & - & -1 & - & - & - & & Pas de lésion & Pas de lésion & - & - \\
\hline 309 & $\mathrm{AD}$ & $>20$ ans & {$[20+]$} & I & - & - & - & - & - & 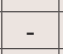 & - & - & - & - & - & $-{ }_{-}^{-}-$ & $-{ }_{-}^{-}$ & - & - & - & - & -- & -- & - & - & - & - & $-{ }_{-}^{-}$ & -- & - & - & - & - & - & - & - & - & - & \\
\hline 310 & $\mathrm{AD}$ & $20-30$ ans & {$[20-29]$} & $\mathrm{M}$ & & 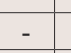 & & - & - & - & - & - & - & - & - & $-{ }_{-}^{-}-$ & - & - & - & 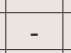 & - &.- & -- & - & - & - & - & $-{ }_{-}^{-}$ & -- & - & 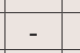 & 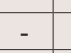 & - & - & & & & & \\
\hline 311 & & & {$[1-4]$} & & - & - & - & - & - & 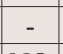 & - & - & - & $=5$ & - & $-\quad-$ & $-{ }_{-}^{-}-$ & - & - & - & & -- & $-\quad-$ & - & - & - & - & $--_{-}^{-}$ & $-\quad-$ & - & - & - & - & - & $\mathrm{NO}$ & résenc & résen, & on & is de lésion \\
\hline 314 & $\mathrm{AI}$ & & {$[30+]$} & $\mathrm{M}$ & & & & - & NO & NO & NO & - & B & NO & B & \begin{tabular}{l|l}
$\mathrm{NO}$ & $\mathrm{NC}$ \\
\end{tabular} & IO $\mathrm{B}$ & & - & & NO N & NO - & - - - & NO & NO & NO & NO & & O - & 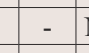 & $\mathrm{NO} /$ & NO & 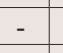 & B & arde lé & & & & \\
\hline 315 & $\mathrm{AI}$ & $>40$ ans & {$[30+]$} & $\mathrm{M}$ & - & $\mathrm{C}$ & & - & - & NO & NO & NO & NO & NO & B & \begin{tabular}{l|l}
$\mathrm{NO}$ & $\mathrm{NC}$ \\
\end{tabular} & $\mathrm{NO} \mathrm{NO}$ & $\mathrm{NO}$ & - & - & $\mathrm{NO} N$ & $\mathrm{NO}$ NG & IO NO & $\mathrm{NO}$ & NO & NO & NO N & NO NO & $\begin{array}{ll}\mathrm{I} & \mathrm{C} \\
\end{array}$ & B & $A$ I & NO & - & $\mathrm{C}$ & as de lésion & Pas de lésion & Pas de lésion & & - \\
\hline 316 & $\mathrm{AD}$ & $>20$ ans & {$[20+]$} & I & - & 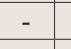 & - & - & - & 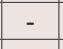 & - & - & -1 & 2 & & \begin{tabular}{l|l}
- & - \\
\end{tabular} & 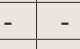 & - & - & - & 2 t & -- & $\begin{array}{l}- \\
-\end{array}$ & - & - & - & - & 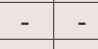 & 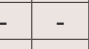 & 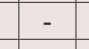 & - & 2 & - & & & & & Pas de lésion & \\
\hline 317 & $\mathrm{AD}$ & $20-30$ ans & {$[20-29]$} & $\mathrm{M}$ & & & & - & - & & & - & 5 & & & 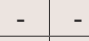 & & & - & & - & 5 & 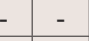 & 5 & 5 & $-a_{0}$ & 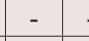 & 5 & 5 & 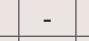 & 5 & 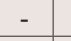 & - & 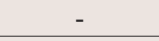 & & & & & \\
\hline 323 & Al & $>20$ ans & {$[20+]$} & I & - & - & - & - & - & - & - & - & - & - & - & \begin{tabular}{l|l}
- & - \\
\end{tabular} & $-1-$ & - & - & - & - & -- & $\begin{array}{l}- \\
-\end{array}$ & - & - & - & $-{ }^{-}$ & \begin{tabular}{l|l}
- & - \\
-
\end{tabular} & \begin{tabular}{l|l}
- & - \\
\end{tabular} & - & - & - & - & 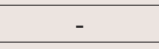 & - & - & - & & \\
\hline 327 & $\mathrm{AD}$ & $30-4$ & {$[30+]$} & I & - & - & - & - & - & - & - & - & - & - & - & \begin{tabular}{l|l}
- & - \\
\end{tabular} & - & - & - & - & - & -- & \begin{tabular}{l|l}
- & - \\
\end{tabular} & - & - & - & $-{ }^{-}-1$ & \begin{tabular}{l|l}
- & - \\
-
\end{tabular} & \begin{tabular}{l|l}
- & - \\
\end{tabular} & - & - & - & - & & & & & Pas de lésion & Pas de lésion \\
\hline 328 & & $0-3$ & {$[0]$} & I & - & - & - & - & - & - & - & - & - & - & - & \begin{tabular}{l|l}
- & - \\
\end{tabular} & - & - & - & - & - & $--_{-}^{-}$ & $\begin{array}{l}- \\
-\end{array}$ & - & - & - & $-{ }^{-}$ & $--_{-}^{-}$ & \begin{tabular}{l|l}
- & - \\
\end{tabular} & - & - & - & - & - & & Pas de lésion & Pas de lésion & & Pas de lésion \\
\hline 329 & & & {$[0]$} & I & - & & - & - & - & - & - & - & - & - & - & $-{ }_{-}^{-}-$ & - & - & - & - & - & \begin{tabular}{l|l}
- & - \\
-
\end{tabular} & $-{ }_{-}^{-}-$ & - & - & - & $-{ }_{-1}$ & $-{ }_{-}^{-}$ & $--_{-}^{-}$ & - & - & - & - & - & as de lésion & & as de lésion & & \\
\hline 330 & & & {$[20+]$} & $\mathrm{M}$ & & 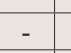 & & - & - & - & & - & - & & & $-{ }_{-}^{-}-$ & - & & - & - & & \begin{tabular}{l|l}
- & - \\
\end{tabular} & -- & - & - & - & - & $-{ }_{-}^{-}$ & -- & - & - & 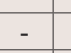 & - & 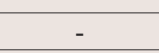 & - & - & s & is de lésion & \\
\hline 331 & & & {$[20+]$} & & - & & & - & - & E & & - & - & & & \begin{tabular}{l|l}
- & - \\
\end{tabular} & - & - & - & - & & \begin{tabular}{l|l}
- & - \\
-
\end{tabular} & \begin{tabular}{l|l}
- & - \\
\end{tabular} & & - & - & -1. & & - & - & - & 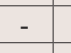 & - & & & & 2 & & \\
\hline 332 & $\mathrm{AI}$ & & {$[30+$} & & & & & - & - & & & - & - & & & \begin{tabular}{l|l}
- & - \\
\end{tabular} & - & & $t_{1}$ & & & - & $\begin{array}{l}- \\
-\end{array}$ & & & - & & & - & & & & & & & & & mode & $\mathrm{m}$ \\
\hline $333 a$ & $\mathrm{AI}$ & ans & {$[20+]$} & $\mathrm{M}$ & - & - & - & - & - & - & - & - & - & - & - & $-{ }_{-}^{-}-$ & - & - & - & - & - & $--_{-}^{-}$ & $--_{-}^{-}$ & - & - & - & - & - & - & - & - & - & - & - & 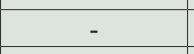 & - & - & - & \\
\hline $333 \mathrm{~b}$ & IN & ans & {$[10-14]$} & 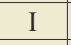 & - & 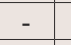 & - & - & - & - & - & - & - & - & - & \begin{tabular}{l|l}
- & - \\
\end{tabular} & - & - & - & - & - & \begin{tabular}{l|l}
- & - \\
-
\end{tabular} & $--_{-}^{-}$ & - & - & - & $-{ }^{-}$ & $--_{-}^{-}$ & $--_{-}^{-}$ & - & - & - & - & - & 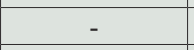 & - & 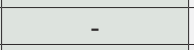 & & \\
\hline 334 & IM & $0-3$ & [0] & I & & & & & & & & & & & & & & & & & & & & & & & & & & & & & & & NO & NO & - & Pas de lésion & Pas de lésion \\
\hline 335 & & & {$[30+]$} & & - & $\mathrm{NO}-\mathrm{r}$ & No & - & - & & - & - & - & & No 1 & $\mathrm{NO} / \mathrm{NC}$ & $\mathrm{NO}$ NO & No & -1 & NO & $\mathrm{NO} / \mathrm{N}$ & NO NO & $\begin{array}{ll}\mathrm{O} \\
\mathrm{O}\end{array}$ & C & NO & No 1 & No $N$ & $\begin{array}{lll}\text { NO } & \text { B } \\
\end{array}$ & \begin{tabular}{l|l}
$B$ & $A$ \\
\end{tabular} & A & NO $\mathrm{T}$ & NO & -1 & C & & NO & & - & - \\
\hline 337 & & & {$[30$} & M: & & NO 1 & No & NO & & NO & & & & & & \begin{tabular}{l|l}
- & - \\
\end{tabular} & $-\mathrm{NO}^{-\mathrm{NO}}$ & & - & & NO & $-\mathrm{NO}$ & $\mathrm{O}$ NO & No & NO & NO 1 , & No $N$ & $\mathrm{NO}$ NO & $\mathrm{O}$ NO & NO & NO $\mathrm{I}$ & NO & & & Pas de lésion & \begin{tabular}{|l} 
Pas de lésion \\
\end{tabular} & \begin{tabular}{|l|} 
Pas del lésion \\
\end{tabular} & & \\
\hline 338 & II & & {$[5-9$} & & & & A & & - & & A & A & B & A & & \begin{tabular}{l|l}
- & - \\
\end{tabular} & $\begin{array}{l}-\mathrm{A} \\
-\end{array}$ & & - & - & & \begin{tabular}{l|l}
$A$ & - \\
\end{tabular} & $\begin{array}{l}- \\
-\end{array}$ & - & A & - & $\begin{array}{ll}\mathrm{B} & \mathrm{f} \\
\end{array}$ & $\begin{array}{ll}\text { A } & - \\
\end{array}$ & \begin{tabular}{l|l}
- & - \\
\end{tabular} & - & A & & - & $\mathrm{B}$ & Présence & Présence & Présence & NO & NO \\
\hline 339 & & & {$[20$} & & - & - & - & - & - & - & - & - & - & & - & $-{ }_{-}^{-}-$ & -- & - & - & - & - & $--_{-}^{-}$ & -- & - & - & - & - & $-\quad-$ & $--_{-}^{-}$ & - & - & - & - & & & & & Pas de lésion & Pas de lésion \\
\hline 340 & $\mathrm{AI}$ & 40 & {$[30+]$} & $\mathrm{M}$ & & 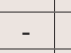 & -5 & $t_{1}$ & - & 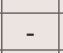 & S & & - & & & $-{ }_{-}^{-}-$ & - & - & - & NO & & NO NO & $\mathrm{O}$ NO & NO & NO & NO & NO N & NO NO & $\mathrm{NO}$ & $\mathrm{NO}$ & -1 & NO & & & $\mathrm{NC}$ & $s$ de lés & as de lésion & 1 & , \\
\hline 341 & & & {$[20+]$} & $\mathrm{F}$ & & - & - & - & - & - & & - & - & & 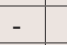 & $-{ }_{-}^{-}-$ & - & -1 & - & - & {$[t$} & \begin{tabular}{l|l}
- & - \\
-
\end{tabular} & $\begin{array}{l}- \\
-\end{array}$ & - & - & St & $-{ }^{-}$ & $-\quad-$ & $--_{-}^{-}$ & & & - & & & & & & in & in \\
\hline 342 & $\mathrm{~A}$ & & {$[20$} & 10 & B & NO & & B & B & B & B & B & A & NO & B & \begin{tabular}{l|l} 
B & B \\
\end{tabular} & B - & NO & NO & A & A 1 & \begin{tabular}{l|l}
$\mathrm{A}$ & $\mathrm{A}$ \\
\end{tabular} & \begin{tabular}{l|l}
$A$ & $A$ \\
\end{tabular} & B & & B & - & - & B & A & NO & & $\mathrm{N}$ & & & & & & \\
\hline 344 & IN & 17 & {$[15-19]$} & $\mathrm{M}$ & A & A & A & $\mathrm{A}$ & A & B & NO & $\mathrm{A}$ & $\mathrm{NO}$ & NO & B & $\begin{array}{ll}\text { A } & \text { A } \\
\end{array}$ & \begin{tabular}{l|l}
$A$ & NO
\end{tabular} & NO & A & A & A 1 & A $\mathrm{A}$ & A $B$ & $\mathrm{C}$ & NO 1 & NO 1 & $\mathrm{NO} \mathrm{N}$ & \begin{tabular}{l|l} 
NO & $\mathrm{C}$ \\
\end{tabular} & \begin{tabular}{|l|l|}
$\mathrm{C}$ & $\mathrm{B}$ \\
\end{tabular} & A & B & A & A & $\mathrm{C}$ & Pas de lésion & Pas de lésion & Pas de lésion & as de lésion & as de lésion \\
\hline 345 & At & & {$[30$} & & - & & & - & - & & & & - & & & - & - & & & & & & - & & - & - & $-{ }_{-1}$ & \begin{tabular}{l|l}
- & - \\
\end{tabular} & -5 & - & & & & 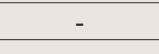 & & & & - & \\
\hline 346 & $\mathrm{Al}$ & & {$[20-$} & 12 & & & & & & & & & & & & & & & & & & & & & & & & & & & & & & & & & & & \\
\hline 347 & & & [20-29] & $\mathrm{M}$ & $\mathrm{A}$ & A & NO & A & A & NO & $\mathrm{A}$ & B & - & $\mathrm{A}$ & $\mathrm{B}$ & \begin{tabular}{l|l} 
A & A \\
\end{tabular} & \begin{tabular}{l|l}
$A$ & $B$ \\
\end{tabular} & A & A & - & $\mathrm{A}$ & \begin{tabular}{l|l} 
A & A \\
\end{tabular} & \begin{tabular}{l|l|}
$A$ & $B$ \\
\end{tabular} & - & B & B & \begin{tabular}{|l|l} 
B & I \\
\end{tabular} & \begin{tabular}{l|l} 
B & B \\
\end{tabular} & \begin{tabular}{|l|l|}
3 & $\mathrm{~A}$ \\
\end{tabular} & - & A & $\mathrm{A}$ & A & B & de lésion & Pas de lésion & as de lés & - & - \\
\hline 348 & IN & & {$[0$} & I & & & & & & & & & & & & & & & & & & & & & & & & & & & & & & & $\mathrm{s}$ de lésion & Pas de lésion & as de lés & - & \\
\hline 349 & $\mathrm{AI}$ & 30 & {$[30+]$} & $\mathrm{M}$ & $\mathrm{A}$ & $\mathrm{B}$ & B & B & NO & A & & - & - & A & A & \begin{tabular}{l|l}
$\mathrm{A}$ & $\mathrm{A}$ \\
\end{tabular} & $\begin{array}{ll}\mathrm{A} & \mathrm{NO} \\
\end{array}$ & $\mathrm{NO}$ & NO & A & $\begin{array}{ll}\text { B } \\
\text { C }\end{array}$ & \begin{tabular}{l|l}
$\mathrm{C}$ & $\mathrm{A}$ \\
\end{tabular} & \begin{tabular}{l|l|}
$A$ & $B$ \\
\end{tabular} & B & B & & \begin{tabular}{l|l}
- & 1 \\
- &
\end{tabular} & \begin{tabular}{l|l}
$\mathrm{B}$ & $\mathrm{NO}$ \\
\end{tabular} & \begin{tabular}{l|l|}
$\mathrm{O}$ & $\mathrm{A}$ \\
\end{tabular} & A & B & B & & $c$ & Pas de lésion & Pas de lésion & Pas de lésion & - & - \\
\hline 350 & IM & $15-$ & {$[15-19]$} & M? & & - & t & - & -1 & 3 & - & - & - & - & 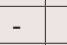 & $-{ }_{-}^{-}-$ & $--_{-}^{-}$ & - & 2 & - & - & $--_{-}^{-}$ & $--_{-}^{-}$ & - & 20 & - & $-{ }_{-1}$ & 2 & - & 2 & 5 & - & - & & & & & - & - \\
\hline 351 & $\mathrm{AI}$ & $25-50 \mathrm{a}$ & {$[20+]$} & I & & - & & t & - & - & - & - & - & - & - & \begin{tabular}{l|l}
- & - \\
\end{tabular} & - & - & - & - & - & - & -1. & - & - & - & - & - - - & - & - & S & - & & . & & & & -5 & \\
\hline 352 & $\mathrm{~A}$ & 20 & [20-29] & $\mathrm{M}$ & $\mathrm{A}$ & B & A & A & A & & B & B & B & & \begin{tabular}{l|l}
$B$ & \\
\end{tabular} & \begin{tabular}{l|l}
$\mathrm{NO}$ & $\mathrm{NC}$
\end{tabular} & No $\mathrm{C}$ & A & A & & NO & B & \begin{tabular}{l|l}
$B$ & NO \\
\end{tabular} & 5 & B & B & B & \begin{tabular}{l|l} 
B & NO \\
\end{tabular} & \begin{tabular}{|l|l|}
$\mathrm{O}$ & $\mathrm{A}$ \\
\end{tabular} & & & B & & & ssion & is $\mathrm{d}$ & as de lésion & & \\
\hline 35 . & & & {$[20-$} & & & 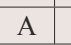 & & A & NO & $\mathrm{NO}$ & NO & B & NO & NO & B & \begin{tabular}{l|l}
$\mathrm{NO}$ & $\mathrm{NC}$
\end{tabular} & vo & & & & A & NO & B & B & B & NO & NO & \begin{tabular}{l|l} 
NO & $B$ \\
\end{tabular} & 2 & & & $\mathrm{NO}$ & & & & & & & \\
\hline 35 & & & & M & & $\mathrm{A}$ & B & A & B & & C & & $\mathrm{C}$ & C & & \begin{tabular}{l|l} 
NO & A
\end{tabular} & $\begin{array}{ll}\mathrm{A} & \mathrm{B} \\
\end{array}$ & B & $\mathrm{A}$ & B & $\mathrm{B}$ & A & \begin{tabular}{l|l|}
$A$ & $B$ \\
\end{tabular} & & & - & & & B & B & C & A & & & & & & & \\
\hline 35 & & & & I & - & NO & B & NO & NO & B & NO & B & B & NO & $\mathrm{C}$ & \begin{tabular}{l|l}
$\mathrm{B}$ & $\mathrm{NC}$ \\
\end{tabular} & No NO & NO & - & - & & $-x_{-1}$ & -2 & - & NO & - & B & B & \begin{tabular}{|l|l|}
3 & $\mathrm{~B}$ \\
\end{tabular} & A & $\mathrm{C}$ & B & & & & & & & \\
\hline $\mathrm{Imt}$ & $\mathrm{AD}$ & & {$[20-2$} & M & & A & A & A & A & A & & A & - & & A & \begin{tabular}{l|l}
$\mathrm{A}$ & $\mathrm{A}$ \\
\end{tabular} & $\begin{array}{ll}\mathrm{A} & \mathrm{A}\end{array}$ & A & & & & & & & & & & & - & - & & & & A & résence & Présence & Presenc & & \\
\hline int 2 & $\mathrm{AL}$ & $20-30$ ans & {$[20-29]$} & $\mathrm{F}$ & - & - & 2 & - & - & - & - & - & - & - & 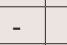 & - - - & 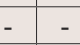 & - & - & - & & & $-1-$ & - & & - & - & & - & - & - & & - & - & 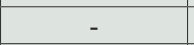 & - & - & Pas de Iesion & \\
\hline int 3 & $\mathrm{AD}$ & $40-60$ ans & {$[30+]$} & M? & & & & -1 & & & & - & - & & & $-\quad-$ & & & - & - & & $-1=$ & & & & & - & & & & & & & & & & & Remodelée & Remodelée \\
\hline
\end{tabular}

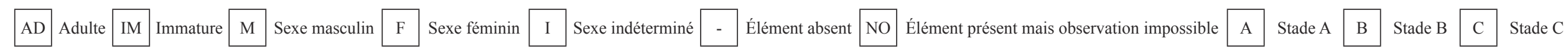


Annexe 3. Données métriques individuelles pour les sujets adultes des échantillons de Sedlec et de la Cathédrale (téléchargeable sur https://journals.openedition.org/bmsap/7744?file=1)

\begin{tabular}{|c|c|c|c|c|c|c|}
\hline Squelette & Sexe & $\begin{array}{c}\text { L max } \\
\text { Humérus }\end{array}$ & $\begin{array}{c}\text { L max } \\
\text { Radius }\end{array}$ & $\begin{array}{c}\text { L max } \\
\text { Ulna }\end{array}$ & $\begin{array}{c}\text { L max } \\
\text { Fémur }\end{array}$ & $\begin{array}{c}\text { L max } \\
\text { Tibia }\end{array}$ \\
\hline $\mathbf{2 0 2}$ & I & 315 & 235 & 255 & 414 & 341 \\
\hline $\mathbf{5 8 5}$ & I & 291 & - & - & - & - \\
\hline $\mathbf{6 0 8}$ & I & - & - & - & - & - \\
\hline $\mathbf{6 1 0}$ & I & 324 & 231 & 257 & - & - \\
\hline $\mathbf{6 2 5}$ & I & - & - & - & - & - \\
\hline $\mathbf{6 2 7}$ & I & 269 & 239 & 255 & - & - \\
\hline $\mathbf{6 4 4}$ & M & - & - & - & - & - \\
\hline $\mathbf{7 5 8 b}$ & I & - & - & - & - & - \\
\hline $\mathbf{7 6 0}$ & M & 323 & 248 & 277 & 438 & - \\
\hline $\mathbf{7 7 7}$ & M & 305 & 224 & 244 & - & - \\
\hline $\mathbf{7 7 8}$ & M & 315 & 239 & - & - & - \\
\hline $\mathbf{8 0 0}$ & I & - & - & - & - & - \\
\hline $\mathbf{8 0 9}$ & M & 330 & 245 & 254 & - & - \\
\hline $\mathbf{8 1 0}$ & I & - & 245 & 263 & - & - \\
\hline $\mathbf{8 2 0}$ & I & - & - & - & - & - \\
\hline $\mathbf{8 2 5}$ & I & 319 & 226 & 250 & 419 & - \\
\hline $\mathbf{8 2 6}$ & I & - & 246 & 267 & - & - \\
\hline $\mathbf{8 4 2}$ & I & - & - & - & 443 & 358 \\
\hline $\mathbf{8 6 4}$ & F & - & - & - & - & - \\
\hline $\mathbf{8 6 9}$ & F & 289 & 217 & 236 & 402 & - \\
\hline $\mathbf{9 0 0}$ & M & - & 240 & 260 & 447 & - \\
\hline $\mathbf{9 0 4}$ & M & - & 246 & 264 & 458 & 368 \\
\hline $\mathbf{1 2 4 9}$ & F & 335 & 252 & 269 & - & - \\
\hline $\mathbf{1 2 5 0}$ & M & 332 & 246 & 263 & 480 & - \\
\hline $\mathbf{1 2 5 2}$ & F & 280 & 216 & 232 & - & - \\
\hline $\mathbf{1 3 1 0}$ & I & - & - & - & - & - \\
\hline $\mathbf{1 3 1 1}$ & F & - & - & - & 406 & - \\
\hline $\mathbf{1 3 1 6}$ & I & - & - & - & - & - \\
\hline $\mathbf{1 3 2 5}$ & M & 313 & 239 & 254 & 453 & 354 \\
\hline $\mathbf{1 3 4 6}$ & M & - & 260 & 282 & 474 & - \\
\hline $\mathbf{1 3 4 8}$ & M & 318 & 240 & 263 & 454 & 350 \\
\hline $\mathbf{1 3 6 2}$ & M & 309 & 227 & 247 & 402 & 324 \\
\hline $\mathbf{1 3 6 4}$ & M & 330 & 253 & 278 & 429 & 352 \\
\hline & & & & & & \\
\hline
\end{tabular}

\begin{tabular}{|c|c|c|c|c|c|c|}
\hline Squelette & Sexe & $\begin{array}{c}\text { L max } \\
\text { Humérus }\end{array}$ & $\begin{array}{c}\text { L max } \\
\text { Radius }\end{array}$ & $\begin{array}{c}\text { L max } \\
\text { Ulna }\end{array}$ & $\begin{array}{c}\text { L max } \\
\text { Fémur }\end{array}$ & $\begin{array}{c}\text { L max } \\
\text { Tibia }\end{array}$ \\
\hline $\mathbf{1}$ & M & - & - & - & 442 & 376 \\
\hline $\mathbf{3}$ & M & 352 & - & - & 500 & - \\
\hline $\mathbf{4}$ & M & - & - & - & - & 369 \\
\hline $\mathbf{5}$ & M & 321 & 248 & 271 & 459 & 359 \\
\hline $\mathbf{1 0}$ & M & 343 & 260 & 282 & - & - \\
\hline $\mathbf{1 5}$ & M & 340 & 240 & 260 & - & - \\
\hline $\mathbf{1 6}$ & M & - & 237 & - & 440 & - \\
\hline $\mathbf{2 3}$ & M & - & - & - & 455 & - \\
\hline $\mathbf{2 5}$ & M & 336 & 245 & 269 & 472 & 375 \\
\hline $\mathbf{2 7}$ & F & 318 & - & - & - & - \\
\hline $\mathbf{2 9}$ & F & 315 & 240 & 262 & - & - \\
\hline $\mathbf{3 1}$ & F & 315 & 226 & 250 & 424 & 365 \\
\hline $\mathbf{3 3}$ & F & - & 230 & - & 414 & 352 \\
\hline $\mathbf{3 8}$ & M & 343 & 250 & 271 & - & - \\
\hline $\mathbf{4 0}$ & M & - & 253 & - & 458 & - \\
\hline $\mathbf{4 1}$ & M & 296 & 221 & 240 & 432 & 340 \\
\hline $\mathbf{5 6}$ & M & - & 246 & 269 & 453 & 360 \\
\hline $\mathbf{6 1}$ & F & - & 238 & 255 & 430 & 352 \\
\hline $\mathbf{6 8}$ & M & - & 271 & 294 & 467 & 375 \\
\hline $\mathbf{8 6}$ & M & 364 & 275 & 294 & - & - \\
\hline $\mathbf{8 9}$ & F & 287 & - & - & 400 & - \\
\hline $\mathbf{9 0}$ & F & 283 & 210 & 229 & - & - \\
\hline $\mathbf{1 0 1}$ & M & 341 & 263 & - & - & - \\
\hline $\mathbf{1 1 1}$ & F & - & 245 & 260 & - & - \\
\hline $\mathbf{1 1 5}$ & M & - & 253 & 279 & 489 & - \\
\hline $\mathbf{1 1 6}$ & F & 310 & - & 242 & - & - \\
\hline $\mathbf{1 1 7}$ & F & 309 & 220 & 240 & 421 & - \\
\hline $\mathbf{1 1 8}$ & M & 348 & 271 & 291 & - & - \\
\hline $\mathbf{1 2 3}$ & F & - & - & - & 415 & 336 \\
\hline $\mathbf{1 2 6}$ & F & 290 & 221 & 238 & 403 & 337 \\
\hline $\mathbf{1 2 7}$ & M & 347 & 255 & 280 & 460 & 385 \\
\hline $\mathbf{1 3 3}$ & M & 343 & 267 & 285 & - & - \\
\hline $\mathbf{1 3 4}$ & M & 359 & 242 & 261 & 464 & - \\
\hline $\mathbf{1 4 1}$ & M & 307 & 230 & 251 & - & - \\
\hline $\mathbf{1 4 3}$ & M & - & 269 & - & 479 & 390 \\
\hline $\mathbf{1 4 4}$ & F & 310 & 225 & 245 & - & - \\
\hline $\mathbf{1 4 5}$ & M & - & - & - & 504 & 409 \\
\hline $\mathbf{1 5 5}$ & M & - & 244 & 269 & 454 & 364 \\
\hline $\mathbf{1 6 0}$ & M & 317 & 231 & 252 & - & - \\
\hline $\mathbf{1 6 2}$ & M & 333 & 260 & - & 459 & - \\
\hline $\mathbf{1 6 3}$ & F & 294 & 215 & 234 & 408 & 337 \\
\hline $\mathbf{1 6 7}$ & M & - & - & - & 443 & \\
\hline $\mathbf{1 7 2}$ & M & 348 & - & - & - & - \\
\hline $\mathbf{1 7 4}$ & M & - & 253 & 279 & 463 & - \\
\hline & & & & & & \\
\hline
\end{tabular}

\begin{tabular}{|c|c|c|c|c|c|c|}
\hline Squelette & Sexe & $\begin{array}{c}\text { L max } \\
\text { Humérus }\end{array}$ & $\begin{array}{c}\text { L max } \\
\text { Radius }\end{array}$ & $\begin{array}{c}\text { L max } \\
\text { Ulna }\end{array}$ & $\begin{array}{c}\text { L max } \\
\text { Femur }\end{array}$ & $\begin{array}{c}\text { L max } \\
\text { Tibia }\end{array}$ \\
\hline $\mathbf{1 7 8}$ & M & 329 & 242 & - & - & - \\
\hline $\mathbf{1 8 0}$ & M & - & - & - & 456 & 365 \\
\hline $\mathbf{1 8 7}$ & F & 299 & 219 & 240 & 413 & - \\
\hline $\mathbf{1 8 9}$ & F & 320 & 237 & 252 & 461 & 362 \\
\hline $\mathbf{1 9 0}$ & M & 331 & 249 & 270 & 451 & 384 \\
\hline $\mathbf{1 9 6}$ & M & 333 & - & - & - & - \\
\hline $\mathbf{1 9 8}$ & M & 323 & - & - & - & - \\
\hline $\mathbf{1 9 9}$ & M & - & 254 & - & - & - \\
\hline $\mathbf{2 0 0}$ & F & - & 243 & 259 & 450 & 375 \\
\hline $\mathbf{2 0 1}$ & M & - & - & - & 436 & 345 \\
\hline $\mathbf{2 0 2}$ & M & 314 & 237 & 255 & 437 & 355 \\
\hline $\mathbf{2 0 3}$ & M & - & - & - & 478 & 372 \\
\hline $\mathbf{2 0 9}$ & M & 338 & 249 & 269 & 463 & - \\
\hline $\mathbf{2 1 0}$ & F & 318 & 238 & 258 & 433 & - \\
\hline $\mathbf{2 1 4}$ & F & - & 236 & 254 & - & - \\
\hline $\mathbf{2 1 6}$ & M & 321 & - & - & - & - \\
\hline $\mathbf{2 2 0}$ & M & - & - & - & 469 & 389 \\
\hline $\mathbf{2 2 1}$ & M & 348 & 259 & 276 & 460 & - \\
\hline $\mathbf{2 2 2}$ & M & 361 & - & - & - & - \\
\hline $\mathbf{2 3 4}$ & M & - & 239 & 258 & - & - \\
\hline $\mathbf{2 3 5}$ & M & 332 & - & 268 & - & - \\
\hline $\mathbf{2 3 9}$ & M & 338 & 264 & 284 & 449 & - \\
\hline $\mathbf{2 5 0}$ & M & 340 & 245 & 265 & 462 & - \\
\hline $\mathbf{2 5 7}$ & M & 342 & 248 & 270 & - & - \\
\hline $\mathbf{2 9 3}$ & M & 332 & 246 & - & - & - \\
\hline $\mathbf{2 9 4}$ & M & - & - & 266 & - & - \\
\hline $\mathbf{2 9 5}$ & M & - & - & - & 450 & - \\
\hline $\mathbf{3 0 5}$ & F & - & 229 & 249 & - & - \\
\hline $\mathbf{3 1 0}$ & M & 334 & 247 & - & - & - \\
\hline $\mathbf{3 0 6}$ & F & - & - & - & 404 & 347 \\
\hline $\mathbf{3 1 4}$ & M & 332 & 244 & 263 & 447 & - \\
\hline $\mathbf{3 1 7}$ & M & - & 261 & 279 & 474 & - \\
\hline $\mathbf{3 3 0}$ & M & - & - & 263 & 463 & 381 \\
\hline $\mathbf{3 3 1}$ & M & - & - & - & 477 & - \\
\hline $\mathbf{3 4 0}$ & M & 345 & 264 & 284 & 470 & - \\
\hline $\mathbf{3 4 1}$ & F & - & - & - & 421 & 363 \\
\hline $\mathbf{3 4 2}$ & M & - & 238 & - & 451 & 360 \\
\hline $\mathbf{3 4 5}$ & M & 319 & - & 258 & 436 & - \\
\hline $\mathbf{3 4 6}$ & M & 324 & - & - & 461 & - \\
\hline $\mathbf{3 4 7}$ & M & 354 & - & 269 & - & - \\
\hline $\mathbf{3 5 2}$ & M & 330 & 236 & 264 & - & - \\
\hline $\mathbf{3 5 3}$ & M & 312 & 238 & - & - & - \\
\hline $\mathbf{3 5 4}$ & M & 309 & 226 & 244 & - & - \\
\hline int1 & F & - & - & 252 & 439 & - \\
\hline & & & & & & \\
\hline
\end{tabular}

